\title{
RAMIFIED AUTOMORPHIC INDUCTION AND ZERO-DIMENSIONAL AFFINE DELIGNE-LUSZTIG VARIETIES
}

\author{
ALEXANDER B. IVANOV
}

\begin{abstract}
To any connected reductive group $G$ over a non-archimedean local field $F$ (of characteristic $p>0$ ) and to any maximal torus $T$ of $G$, we attach a family of extended affine Deligne-Lusztig varieties (and families of torsors over them) over the residue field of $F$. This construction generalizes affine Deligne-Lusztig varieties of Rapoport, which are attached only to unramified tori of $G$. Via this construction, we can attach to any maximal torus $T$ of $G$ and any character of $T$ a representation of $G$. This procedure should conjecturally realize the automorphic induction from $T$ to $G$.

For $G=\mathrm{GL}_{2}$, we prove that our construction indeed realizes the automorphic induction from at most tamely ramified tori. Moreover, if the torus is purely tamely ramified, then the varieties realizing this correspondence are zero-dimensional and reduced, i.e., just disjoint unions of points.
\end{abstract}

\section{INTRODUCTION}

Let $G$ be a connected reductive group over a finite field $\mathbb{F}_{q}$ and let $\sigma$ be the Frobenius over $\mathbb{F}_{q}$. Then there is a natural correspondence, which to any pair $(T, \chi)$ consisting of a maximal $\mathbb{F}_{q}$-torus $T$ of $G$ and a character $\chi$ of $T\left(\mathbb{F}_{q}\right)$ in general position, associates an irreducible representation $R_{T}^{\chi}$ of $G\left(\mathbb{F}_{q}\right)$. Moreover, $R_{T}^{\chi}$ is cuspidal, whenever $T$ is anisotropic modulo the center of $G$. This was conjectured in 1968 by MacDonald and proven in 1976 by Deligne and Lusztig in their celebrated paper $[\mathrm{DL}]$. They defined $R_{T}^{\chi}$ as the alternating sum of the $\ell$-adic cohomology with compact support of an étale cover of a Deligne-Lusztig variety. This last is just the variety of all Borel subgroups of $G$, which are in a fixed relative position with their $\sigma$-translates.

Let now $G$ be a connected reductive group over a non-archimedean local field $F$ with residue field $\mathbb{F}_{q}$ and let $\ell \neq \operatorname{char}\left(\mathbb{F}_{q}\right)$ be a prime. For simplicity (to avoid dealing with endoscopy phenomena, etc.) let us assume here that $G=\mathrm{GL}_{n}$. Then there is a correspondence, similar to the one explained above, which associates to any pair $(T, \chi)$ consisting of a maximal $F$-torus $T$ of $G$ and a smooth $\overline{\mathbb{Q}}_{\ell}^{\times}$-character of $T(F)$ in general position, an irreducible representation $R_{T}^{\chi}$ of $G(F)$ over $\overline{\mathbb{Q}}_{\ell}$. Moreover, $R_{T}^{\chi}$ is supercuspidal, whenever $T$ is anisotropic modulo the center of $G$. Such a correspondence is a special case of the more general principle of automorphic induction for $G$, which is closely related to the local Langlands correspondence.

Let $G$ again be arbitrary. Roughly, one can divide all geometrical objects attached to $G$, in the cohomology of which one has tried to realize the automorphic induction, into two types:

(i) Varieties (or rigid or adic spaces) over $\operatorname{Spec} F$ equipped with integral models over $\operatorname{Spec} \mathcal{O}_{F}$ and special fibers over $\mathbb{F}_{q}$.

(ii) Reduced varieties over $\mathbb{F}_{q}$.

Constructions of type (ii) are purely in characteristic $p$, i.e., over $\mathbb{F}_{q}$, and only the reduced structure is relevant. Up to now, constructions of type (ii) only existed for unramified tori of

2010 Mathematics Subject Classification. 11S37, 14M15, 11F70.

Key words and phrases. affine Deligne-Lusztig variety, automorphic induction, local Langlands correspondence, supercuspidal representations.

The author was partially supported by ERC starting grant 277889 "Moduli spaces of local G-shtukas". 
$G$ (except for a construction by Stasinski for $\mathrm{GL}_{n}$ and $\mathrm{SL}_{n}$, see below), which was a serious drawback. This article contributes to the automorphic induction over local fields by introducing a new construction of type (ii), which works for all tori and all reductive groups (in the equal characteristic case). For $G=\mathrm{GL}_{2}$ we prove that our construction indeed realizes the $\ell$-adic automorphic induction for all at most tamely ramified tori. Here a very intriguing phenomenon occurs: the constructed varieties attached to the tamely ramified torus of $\mathrm{GL}_{2}$ turn out to be zero-dimensional and reduced, more precisely, they are just discrete unions of $\mathbb{F}_{q}$-rational points and the automorphic induction is realized in their zeroth cohomology groups $\mathrm{H}_{c}^{0}\left(-, \overline{\mathbb{Q}}_{\ell}\right)$ with coefficients in the constant sheaf $\overline{\mathbb{Q}}_{\ell}$.

We recall some of the existing unramified constructions of type (ii). A first such construction was suggested in 1977 by Lusztig [Lu]. Its variants were studied by Boyarchenko, BoyarchenkoWeinstein and Chan in [Bo], $[\mathrm{BW}],[\mathrm{Ch}]$. A different, but apparently related approach via higher level covers of Rapoport's affine Deligne-Lusztig varieties was studied by the author in [Iv2] (in the equal characteristic case). The nature of all these constructions is strongly related to the classical Deligne-Lusztig construction explained in the beginning. In particular, if $\breve{F}$ denotes the completion of the unramified closure of $F, \sigma$ the Frobenius of $\breve{F} / F$, and $b \in G(\breve{F})$ is some element, then an affine Deligne-Lusztig variety attached to these data can be seen as the subvariety of the affine flag manifold of $G$, consisting of all Iwahori subgroups of $G(\breve{F})$ being in a fixed relative position with their $b \sigma$-translates.

Main construction. We will define the extended affine Deligne-Lusztig varieties and torsors naturally attached to them in Section 2 below. Roughly, the construction goes as follows. Let $F=\mathbb{F}_{q}((t))$ be a local field of characteristic $p$. Let $G$ be a connected reductive group over $F$. Let $\mathfrak{T}$ be a maximal $F$-torus of $G$. Let $\breve{E} / F$ be the completion of the maximal unramified extension of the splitting field $E$ of $\mathfrak{T}$ and let $\Sigma$ be a set of generators of $\mathrm{Gal}_{\breve{E} / F}$. Let $\underline{w}$ be a map from $\Sigma$ to the set of all possible relative positions of Iwahori subgroups of $G(\breve{E})$ and let $b \in G(\breve{E})$. Then the extended affine Deligne-Lusztig set attached to $\underline{w}$ and $b$ is the subset $X_{\underline{w}}(b)$ of the affine flag manifold $\mathscr{F}$ of $G_{\breve{E}}$ consisting of all Iwahori subgroups, whose relative position to their $b \gamma$-translate is equal to $\underline{w}(\gamma)$ for all $\gamma \in \Sigma$.

Now we turn to torsors of $X_{\underline{w}}(b)$. By a level $f$ we essentially mean a congruence subgroup $I^{f}$

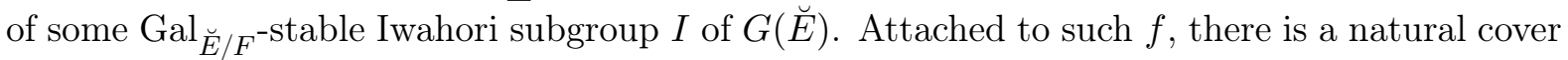
$\mathscr{F} f \rightarrow \mathscr{F}$ of the affine flag manifold. Then to any lift $\underline{w}_{f}$ of $\underline{w}$ to a function into an appropriate space of relative positions of level $f$, we naturally attach a subset $X_{\underline{w}_{f}}^{f}(b)$ of $\mathscr{F} f$, which lies over $X_{\underline{w}}(b)$. In many cases, $X_{\underline{w}}(b)$ and $X_{\underline{w}_{f}}^{f}(b)$ can be given a scheme structure, turning them into reduced schemes locally of finite type over a finite extension of $\mathbb{F}_{q}$. Moreover, we obtain two natural commuting group actions

$$
J_{b}(F) \subset X_{\underline{w}_{f}}^{f}(b) り \tilde{I}_{f, \underline{w}_{f}} \rightarrow \mathfrak{T}(F) .
$$

Here $J_{b}$ is the $\Sigma$-stabilizer of $b$, i.e., the algebraic group over $F$ defined by

$$
J_{b}(R):=\left\{g \in G\left(R \otimes_{F} \breve{E}\right): g^{-1} b \gamma(g)=b \forall \gamma \in \Sigma\right\}
$$

for an $F$-algebra $R$, and $\tilde{I}_{f, \underline{w}_{f}}$ is a certain subgroup of $G(\breve{E})$, which depends on $\underline{w}_{f}$ (but not on $b)$ and admits $\mathfrak{T}(F)$ as a natural quotient, if $\underline{w}_{f}$ is appropriate. Further, $X_{\underline{w}_{f}}^{f}(b)$ is in a natural way a torsor over $X_{\underline{w}}(b)$ under a certain subquotient of $\tilde{I}_{f, \underline{w}_{f}}$, which is an algebraic group of finite type over a finite extension of $\mathbb{F}_{q}$.

The construction explained above generalizes the unramified construction from [Iv2], i.e., if one chooses $\mathfrak{T}$ to be an unramified maximal torus of $G$ (i.e., $\breve{E}=\breve{F}$ ) and $\Sigma$ to be the set with 
one element containing only the Frobenius of $\breve{F} / F$, then the corresponding Iwahori-level variety $X_{\underline{w}}(b)$ will be just the affine Deligne-Lusztig variety of Rapoport, and the torsors $X_{\underline{w}_{f}}^{f}(b)$ over it will be precisely the torsors defined in [Iv2].

We wish to point out that in 2011 Stasinski made in $[\mathrm{St}]$ the first attempt to define some varieties (of type (ii)) attached to ramified tori. He worked over finite rings $\mathbb{F}_{q}[t] /\left(t^{r}\right)$ and was interested in the representation theory of the finite group $G\left(\mathbb{F}_{q}[t] /\left(t^{r}\right)\right)$. For $G=\mathrm{GL}_{n}, \mathrm{SL}_{n}$, he constructed extended Deligne-Lusztig varieties (hence our choice of terminology) attached to $G\left(\mathbb{F}_{q}[t] /\left(t^{r}\right)\right)$ and any maximal torus in $G=\mathrm{GL}_{n}, \mathrm{SL}_{n}$. This construction is technically involved, and, in particular, works a priori only for $G=\mathrm{GL}_{n}, \mathrm{SL}_{n}$. Also, there are issues about defining higher-level torsors. Nevertheless, the main ideas of his and our constructions seem to coincide: in both cases, one defines a variety by fixing the relative position with respect to many automorphisms of an extension of $F$, and not only with respect to the Frobenius. Moreover, the first example of a zero-dimensional variety (attached to $\mathrm{SL}_{2}\left(\mathbb{F}_{q}[t] /\left(t^{2}\right)\right)$ ), realizing interesting representations occurs in $[\mathrm{St}]$.

Affine Deligne-Lusztig induction. We can use the $\ell$-adic cohomology of $X_{\underline{w}_{f}}^{f}(b)$ to define the following map, which we call the affine Deligne-Lusztig induction:

$$
R=R_{f, \underline{w}_{f}, b}: \operatorname{Rep}_{\overline{\mathbb{Q}}_{\ell}}\left(\tilde{I}_{f, \underline{w}_{f}} / I^{f}\right) \rightarrow \operatorname{Rep}_{\overline{\mathbb{Q}}_{\ell}}\left(J_{b}(F)\right), \quad \chi \mapsto \sum_{i}(-1)^{i} \mathrm{H}_{c}^{i}\left(X_{\underline{w}_{f}}^{f}(b), \overline{\mathbb{Q}}_{\ell}\right)[\chi]
$$

between the categories of smooth $\overline{\mathbb{Q}}_{\ell}$-representations. In particular, whenever $\underline{w}_{f}$ is such that $\mathfrak{T}(F)$ is a quotient of $I_{f, \underline{w}_{f}}$, characters of $\mathfrak{T}(F)$ (of level bounded by $f$ ) induce, after inflation to $\tilde{I}_{f, \underline{w}_{f}}$, representations of $J_{b}(F)$. We formulate the following conjecture here only for $\mathrm{GL}_{n}$ and $b=1$, in which case $J_{1}(F)=G(F)$. For more general reductive groups $G$ we expect that a similar statement is true, but that some endoscopy phenomena occur.

Conjecture 1.1. Let $G=\mathrm{GL}_{n}$ and $b=1$. The collection of maps (1.1) satisfies the following properties:

(A) If $\mathfrak{T}$ is anisotropic modulo the center of $G$, and $\chi$ is a character of $\mathfrak{T}(F)$ in sufficiently general position, then there are $f, \underline{w}_{f}$, such that $\tilde{I}_{f, \underline{w}_{f}} \rightarrow \mathfrak{T}(F)$ and $R_{f, \underline{w}_{f}, 1}(\chi)$ is an irreducible supercuspidal representation of $G(F)$.

(B) The map $\chi \mapsto R_{f, \underline{w}_{f}, 1}(\chi)$ in $(A)$ is injective up to Galois conjugation.

(C) The map $\chi \mapsto R_{f, \underline{w}_{f}, 1}(\chi)$ in (A) coincides with the realization of the automorphic induction constructed via cuspidal types by Bushnell, Kutzko and others (see [BK]).

Further, we expect the following two facts to be true:

(D) If $\mathfrak{T}$ is unramified, then $X_{\underline{w}_{f}}^{f}(1)$ is isomorphic (up to a possible unessential defect) to the reduction of the open affinoid in the Lubin-Tate perfectoid space constructed by Boyarchenko and Weinstein in $[\mathrm{BW}]$.

(E) If $\mathfrak{T}$ is purely tamely ramified, then $\underline{w}_{f}$ can be chosen such that $X_{\underline{w}_{f}}^{f}(1)$ is a zerodimensional reduced scheme (disjoint union of points).

The evidence for Conjecture 1.1 and expectations (D) and (E) is build up mainly on the at most tamely ramified $\mathrm{GL}_{2}$-case (see Theorem 1.2 below), and the analogy with the classical Deligne-Lusztig induction. We discuss some further evidence below in this introduction.

Case $G=\mathrm{GL}_{2}$. Let $\mathbb{P}_{2}(F)$ be the set of all isomorphism classes of admissible pairs $(E / F, \chi)$ attached to at most tamely ramified quadratic extensions $E / F$. Note that if $\mathfrak{T} \subseteq G$ is a torus with $\mathfrak{T}(F)=E^{\times}$, then characters of $\mathfrak{T}(F)$ in general position up to Galois conjugation are in 1:1-correspondence with the subset of minimal pairs. Let $\mathscr{A}_{2}^{\text {tame }}(F)$ be the set of isomorphism 
classes of irreducible supercuspidal representations of $G(F)$, which are additionally assumed to be unramified if char $F=2$. Then the tame parametrization theorem ( $[\mathrm{BH}] 20.2$ Theorem) shows the existence of a certain well-behaved bijection

$$
\mathbb{P}_{2}(F) \stackrel{\sim}{\rightarrow} \mathscr{A}_{2}^{\text {tame }}(F), \quad(E / F, \chi) \mapsto \pi_{\chi} .
$$

The following theorem shows Conjecture 1.1 and expectation (E) for $\mathrm{GL}_{2}$ for all at most tamely ramified tori in $\mathrm{GL}_{2}$.

Theorem 1.2 (rough statement; cf. Theorem 4.2 and [Iv2] Theorem 4.3). Let $G=\mathrm{GL}_{2}$. Let $\mathfrak{T}$ be a non-split maximal torus of $G$. Let $\breve{E} / F$ be the completion of the unramified closure of the splitting field $E$ of $\mathfrak{T}$. Then there are choices of $\Sigma \subseteq \mathrm{Gal}_{\breve{E} / F}, f, \underline{w}_{f}$ such that the corresponding maps $R=R_{f, \underline{w}_{f}, 1}$ from (1.1) realize the automorphic induction from $\mathfrak{T}$ to $G$. Moreover, they induce a bijection

$$
\mathbb{P}_{2}(F) \stackrel{\sim}{\rightarrow} \mathscr{A}_{2}^{\text {tame }}(F), \quad(E / F, \chi) \mapsto R_{\chi},
$$

and one has $R_{\chi} \cong \pi_{\chi}$, i.e., the maps 1.3 and 1.2 coincide. In the case of the ramified torus, $\underline{w}_{f}$ can be chosen such that the varieties $X_{\underline{w}_{f}}^{f}(1)$ are disjoint unions of $\mathbb{F}_{q}$-points.

If $\mathfrak{T}$ is tamely ramified with splitting field $E$, it suffices to work with $E$ instead of $\breve{E}$ to obtain the same results (see Remark 3.10(i)). A similar statement is not true if $\mathfrak{T}$ is unramified.

Ramified torus in $\mathrm{GL}_{2}$. Besides the construction explained above, the main result of this article is Theorem 4.2 (the tamely ramified case of Theorem 1.2 the unramified case was proven in Iv2]). Its proof is analogous to the proof in the unramified case [Iv2] Theorem 4.3. Roughly speaking, there are three instruments used in the proof:

(1) a trace formula, which in the present case reduces, due to zero-dimensionality, to (quite involved) point-counting arguments.

(2) the theory of elementary modifications of characters of $E^{\times}$, developed in Section 4.4 . It allows to prove our second main result, Theorem 4.18. It gives a precise description of the restriction of a certain cuspidal type (from which $R_{\chi}$ is induced) to an $E^{\times}$-representation. From this we deduce the injectivity of 1.3 .

(3) the theory of cuspidal types of Bushnell, Kutzko, Henniart and others. We need it to compare $R_{\chi}$ with $\pi_{\chi}$ and, in particular, to show surjectivity of 1.3 .

Some evidence for Conjecture 1.1. Let now $G=\mathrm{GL}_{n}$ and let $\mathfrak{T}$ be a maximal $F$-torus of $G$. Here are some heuristic reasons, justifying Conjecture 1.1 and the expectations (D) and (E):

- If $G=\mathrm{GL}_{2}$ and $\mathfrak{T}$ is at most tamely ramified, (A),(B),(C),(E) hold (see Theorem 4.2 below and [Iv2] Theorem 4.3)

- The construction is completely analogous to the classical Deligne-Lusztig induction.

- (D) becomes evident for $G=\mathrm{GL}_{2}, b$ superbasic by looking at the explicit defining equations (see [Iv2] Section 3.6).

- Assume $\mathfrak{T}$ is unramified, and let $\Sigma:=\{\sigma\}$ consist only of the Frobenius, i.e., the corresponding varieties are torsors of level $f$ over the usual affine Deligne-Lusztig varieties $X_{w}(1)$, where $w$ is an element of the extended affine Weyl group $\tilde{W}$ of $G$. By $\mathrm{GH}$ Proposition 2.2.1, if $w$ is contained in a finite Weyl subgroup of $\tilde{W}$, then essentially, $X_{w}(1) \cong \coprod_{g \in G(F) / G\left(\mathcal{O}_{F}\right)} g X_{w}$, where the union is taken over translates of classical Deligne-Lusztig varieties $X_{w}$. This implies a similar decomposition for level- $f$-covers $X_{\underline{w}_{f}}^{f}(1)=\coprod_{g \in G(F) / G\left(\mathcal{O}_{F}\right)} g Y_{\underline{w}_{f}}^{f}$ with $Y_{\underline{w}_{f}}^{f}$ of finite type. If $Z$ denotes the center of $G(F)$, we deduce

$$
\mathrm{H}_{c}^{*}\left(X_{\underline{w}_{f}}^{f}(1), \overline{\mathbb{Q}}_{\ell}\right)[\chi]=\mathrm{c}-\operatorname{Ind}_{Z G\left(\mathcal{O}_{F}\right)}^{G(F)} \mathrm{H}_{c}^{*}\left(Y_{\underline{w}_{f}}^{f}, \overline{\mathbb{Q}}_{\ell}\right)[\chi]
$$


On the other side, it follows from the theory of cuspidal types (see $[\mathrm{BK}]$ ) that any supercuspidal representation is compactly induction from a cuspidal type, thus we have, in particular, a natural family of supercuspidal representations of $G(F)$, which are all of the form c $-\operatorname{Ind}_{Z G\left(\mathcal{O}_{F}\right)}^{G(F)} \Xi$, where $\Xi$ is some cuspidal inducing datum. Thus if the torus is unramified, the conjecture should boil down to statements about smooth representations of the group $Z G\left(\mathcal{O}_{F}\right)$, which is compact modulo center. For $n=2$, this holds also for the tamely ramified torus and is part of our strategy of the proof of Theorem 1.2 .

- Concerning expectation (E), we remark that if some lift $\sigma^{\prime}$ of the Frobenius $\sigma \in \mathrm{Gal}_{\breve{F} / F}$ lies in $\Sigma$ and $\underline{w}\left(\sigma^{\prime}\right)=1$, then the extended affine Deligne-Luszstig variety $X_{\underline{w}}(1)$ of Iwahori level is a disjoint union of points (for any connected reductive $G$ ).

Outline. In Section 2 we define the extended affine Deligne-Lusztig varieties and their covers for arbitrary connected reductive groups. In Section 3 we compute certain of those varieties for $G=\mathrm{GL}_{2}$ explicitly. Based on these computations, in Section 4, we state and prove our main results about tamely ramified automorphic induction for $\mathrm{GL}_{2}$. The proofs of all results from Section 4, which contain any trace computations, are postponed to Section 5 .

Acknowledgements. The author is very grateful to Christian Liedtke, Stephan Neupert, Peter Scholze and Eva Viehmann for helpful discussions concerning this work. He is especially grateful to Eva Viehmann for valuable comments concerning a preliminary version of this manuscript. The author was partially supported by ERC starting grant 277889 "Moduli spaces of local $G$ shtukas".

\section{Extended affine Deligne-Lusztig varieties of higher Level}

\subsection{Preliminaries.}

2.1.1. Basic notation. Let $k$ be the finite field with $q$ elements and let $\bar{k}$ be an algebraic closure of $k$. Let $F=k((t))$. Let $\breve{F}=\bar{k}((t))$ be the completion of the maximal unramified extension of $F$. Let $E / F$ be a finite separable extension of $F$, such that $\breve{E}:=E \breve{F}$ is the completion of a Galois extension of $F$. We denote by $u$ a uniformizer of $E$ and set $k_{E}=\bar{k} \cap E$ (inside some fixed algebraic closure of $F)$. We have the identifications $E=k_{E}((u))$ and $\breve{E}=\bar{k}((u))$. For a Galois extension $M / L$ we denote by $\mathrm{Gal}_{M / L}$ its Galois group.

2.1.2. Group theoretic data. Let $G$ be a connected reductive group over $F$. Let $S_{0}$ be a maximal split torus in $G_{\breve{F}}$. By [BT2] 5.1.12, $S_{0}$ can be chosen to be defined over $F$. Let $T_{0}:=\mathscr{Z}_{G_{\breve{F}}}\left(S_{0}\right)$ be the centralizer of $S_{0}$. By Steinberg's theorem, $G_{\breve{F}}$ is quasi-split, hence $T_{0}$ is a maximal torus. Then the base change $T:=T_{0} \otimes_{\breve{F}} \breve{E}$ is a maximal torus of $G_{\breve{E}}$. Let $S^{\prime}$ be a maximal $\breve{E}$-split subtorus of $T$, containing $S:=S_{0} \otimes_{\breve{F}} \breve{E}$. We consider the root system $\Phi:=\Phi\left(G_{\breve{E}}, S^{\prime}\right)$. For $a \in \Phi$, we write $U_{a}$ for the corresponding root subgroup of $G_{\breve{E}}$. Moreover, we write $U_{0}:=T$.

2.1.3. Bruhat-Tits buildings. For any finite extension $L$ of $F$ or $\breve{F}$, let $\mathscr{B}_{L}$ denote the BruhatTits building of $G$ over $L$. It always exists by $[\overline{\mathrm{BT}}],[\overline{\mathrm{BT}} 2] 44,[\mathrm{Ro}]$ Chap. 5 and $[\mathrm{MSV}]$. If $L \subseteq M$ are two such extensions such that $M / L$ is Galois, then $\mathrm{Gal}_{M / L}$ acts on $\mathscr{B}_{M}$. Moreover, there is a unique embedding of $\mathscr{B}_{L}$ into $\mathscr{B}_{M}$ in the sense of [Ro] Definition 2.5.1. Indeed, the centralizer $T$ of $S_{0}$ is a maximal torus, hence abelian, and its derived group is trivial. This allows to apply $[\mathrm{Ro}]$ Theorem 2.5.6, to show that there is a unique such embedding. Note that if $M / L$ is ramified, then $\mathscr{B}_{L}, \mathscr{B}_{M}^{\mathrm{Gal}_{M / L}}$ are not equal as simplicial complexes. However, if $M / L$ is Galois tamely ramified, then $\mathscr{B}_{L}=\mathscr{B}_{M}^{\mathrm{Gal}_{M / L}}$ as subsets, as follows from [Ro 5.1 .1 (see also [Pr|). 
2.1.4. Apartments and Galois-stable alcoves. Let $\mathscr{A}_{S^{\prime}}$ be the apartment of $\mathscr{B}_{\breve{E}}$ corresponding to $S^{\prime}$. Via the embedding $\mathscr{B}_{\breve{F}} \hookrightarrow \mathscr{B}_{\breve{E}}$ it contains the apartment $\mathscr{A}_{S_{0}}$ of $\mathscr{B}_{\breve{F}}$ corresponding to $S_{0}$. The restriction of any root $a$ in $\Phi$ to $S$ is non-trivial (indeed, otherwise $U_{a}$ would lie in the centralizer $\mathscr{Z}_{G_{\breve{E}}}(S)$ of $S$, but taking the centralizer commutes with base change, hence $\mathscr{Z}_{G_{\breve{E}}}(S)=\mathscr{Z}_{G_{\breve{F}}}\left(S_{0}\right) \otimes_{F} \breve{F}=T$. This leads to a contradiction). This means that $\mathscr{A}_{S_{0}}$ is not contained in a wall of $\mathscr{A}_{S^{\prime}}$. Take some alcove $\underline{a}$ of $\mathscr{A}_{S^{\prime}}$ which contains a point $x_{0}$ of $\mathscr{A}_{S_{0}}$ in its interior. Then $\mathrm{Gal}_{\breve{E} / \breve{F}}$ fixes $x_{0}$. As $S_{0}$ is defined over $F, \mathrm{Gal}_{\breve{F} / F}$ acts on $\mathscr{A}_{S_{0}}$ and we assume that $x_{0}$ is $\mathrm{Gal}_{\breve{F} / F}$-stable. Then $\underline{a}$ is also $\mathrm{Gal}_{\breve{E} / F^{-}}$-stable. Let $I$ denote the associated $\mathrm{Gal}_{\breve{E} / F^{-}}$stable Iwahori subgroup of $G(\breve{E})$.

2.1.5. Filtrations on root subgroups. Let $x$ be a vertex of $\underline{a}$. Let $\tilde{\mathbb{R}}:=\mathbb{R} \cup\{r+: r \in \mathbb{R}\} \cup\{\infty\}$ be the ordered monoid as in $\mathrm{BT}$ 6.4.1. Then for all $a \in \bar{\Phi}, x$ defines a filtration of $U_{a}(\breve{E})$ by subgroups $U_{a}(\breve{E})_{x, r}$ for $r \in \tilde{\mathbb{R}}$.

2.1.6. Filtration on the torus. We choose an admissible schematic filtration on tori in the sense of $\mathrm{Yu}[\mathrm{Yu}] \S 4$. This gives a filtration $U_{0}(\breve{E})_{r}=T(\breve{E})_{r}$ on $T(\breve{E})$. If $G$ satisfies the condition (T) from $[\mathrm{Yu}$ 4.7.1 (in particular, if $G$ is either simply connected or adjoint or split over a tamely ramified extension), then this filtration is independent of the choice of the admissible schematic filtration and coincides with the Moy-Prasad filtration $\mid \mathrm{Yu} \|$ Lemma 4.7.4.

2.1.7. Smooth models of root subgroups. Let $f: \Phi \cup\{0\} \rightarrow \tilde{\mathbb{R}}_{\geqslant 0} \backslash\{\infty\}$ be a concave function, i.e., $f\left(\sum_{i} a_{i}\right) \geqslant \sum_{i} f\left(a_{i}\right)$, for all $a_{i} \in \Phi \cup\{0\}$, such that $\sum_{i} a_{i} \in \Phi \cup\{0\}$ (see $|\mathrm{BT}| 6.4$ ). Following $|\mathrm{Yu}|$, let $G(\breve{E})_{x, f}$ be the subgroup of $G(\breve{E})$ generated by $U_{a}(\breve{E})_{x, f(a)}$ for $a \in \Phi \cup\{0\}$. We refer to $f$ as a level and to $G(\breve{E})_{x, f}$ as the corresponding level subgroup. By |Yu| Theorem 8.3, there is a unique smooth model $\underline{G}_{x, f}$ of $G_{\breve{E}}$ over $\mathcal{O}_{\breve{E}}$ such that $\underline{G}_{x, f}\left(\mathcal{O}_{\breve{E}}\right)=G(\breve{E})_{x, f}$. Moreover, if $G(\breve{E})_{x, f}$

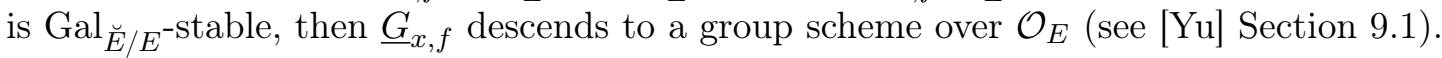

For a concave function $f$, we write $I^{f}:=G(\breve{E})_{x, f}$. Let $f_{I}$ denote the concave function such that $G(\breve{E})_{x, f}=I$. If $f \geqslant f_{I}$, then $I^{f} \subseteq I$.

2.1.8. Loop groups and covers of the affine flag variety. Let $L G$ denote the functor on the category of $k_{E}$-algebras,

$$
L G: R \mapsto G(R((u))) .
$$

Let $f \geqslant f_{I}$ be some level, such that $I^{f}$ is $\mathrm{Gal}_{\breve{E} / E^{- \text {stable. }}}$ Let $L^{+} \underline{G}_{x, f}$ be the functor on the category of $k_{E}$-algebras,

$$
L^{+} \underline{G}_{x, f}: R \mapsto \underline{G}_{x, f}(R \llbracket u \rrbracket) .
$$

Then by $\mathrm{PR}$ Theorem 1.4 the quotient of fpqc-sheaves

$$
\mathscr{F}^{f}:=L G / L^{+} \underline{G}_{x, f}
$$

is represented by an Ind- $k_{E}$-scheme of Ind-finite type over $k_{E}$ and its $\bar{k}$-points are $\mathscr{F}^{f}(\bar{k})=$ $G(\breve{E}) / G(\breve{E})_{x, f}$. Moreover, if $g \geqslant f$ are two concave functions satisfying the above assumptions, then there is a natural projection $\mathscr{F}^{g} \rightarrow \mathscr{F}^{f}$. We write $\mathscr{F}$ instead of $\mathscr{F}^{f_{I}}$. This is just the affine flag manifold of $G$.

2.1.9. Actions on $\mathscr{F} f$. Let $f \geqslant f_{I}$ be some level. By construction, $L G$ acts on $\mathscr{F} f$ by left multiplication. In particular, $G(\breve{E})=L G(\bar{k})$ acts on the $\bar{k}$-valued points $\mathscr{F} f(\bar{k})=G(\breve{E}) / G(\breve{E})_{x, f}$. Assume now that $I^{f}$ is normal in $I$ and let $Z$ be the center of $G$. Then $Z(\breve{E}) I$ acts by right multiplication on $\mathscr{F}^{f}(\bar{k})$. 
2.1.10. Extended affine Weyl group and Iwahori-Bruhat decomposition. Let $\mathscr{N}_{T}$ be the normalizer of $T$ in $G$. Let $\tilde{W}$ be the extended affine Weyl group of $G_{\breve{E}}$ associated with $S^{\prime}$. Let $W:=\mathscr{N}_{T}(\breve{E}) / T(\breve{E})$ be the finite Weyl group. Then $\tilde{W}$ sits in the short exact sequence

$$
0 \rightarrow X_{*}\left(T_{\breve{E}}\right)_{\mathrm{Gal}_{\breve{E}}} \rightarrow \tilde{W} \rightarrow W \rightarrow 0
$$

(here $\mathrm{Gal}_{\breve{E}}$ denotes the absolute Galois group of $\breve{E}$ ). The Iwahori-Bruhat decomposition states that

$$
G(\breve{E})=\coprod_{w \in \tilde{W}} I \dot{w} I
$$

where $\dot{w}$ is any lift of $w$ to $\mathscr{N}_{T}(\breve{E})$.

2.1.11. Double coset decomposition at level $f$. Let $f \geqslant f_{I}$ be some fixed level. Consider the set of double cosets

$$
D_{G_{\breve{E}}, f}:=I^{f} \backslash G(\breve{E}) / I^{f} .
$$

If $g \geqslant f$, then there is a natural projection $D_{G_{\breve{E}}, g} \rightarrow D_{G_{\breve{E}}, f}$. In particular, we have the natural projection $D_{G_{\breve{E}}, f} \rightarrow D_{G_{\breve{E}}, f_{I}}=I \backslash G(\breve{E}) / I \cong \tilde{W}$. For many $w, f$ the fiber $D_{G, f}(w)$ of this projection can be given the structure of a finite-dimensional affine variety over $\bar{k}$. This will be discussed in detail in a future work. Below we only need the case $G=\mathrm{GL}_{2}$, where an explicit parametrization can be given (see Section 3.1.8).

2.1.12. Relative position. Let $f$ be as in Section 2.1.11. We define the map

$$
\operatorname{inv}^{f}: \mathscr{F}^{f}(\bar{k}) \times \mathscr{F}^{f}(\bar{k}) \rightarrow D_{G_{\breve{E}}, f}
$$

on $\bar{k}$-points by $\operatorname{inv}^{f}\left(x I^{f}, y I^{f}\right)=w_{f}$, where $w_{f}$ is the unique double $I^{f}$-coset containing $x^{-1} y$.

\subsection{Extended affine Deligne-Lusztig varieties of higher level.}

\subsubsection{Main definition.}

Definition 2.1. Let $G$ be a connected reductive group over $F$. Fix the following data:

- a finite separable extension $E / F$, such that $\breve{E}=E \breve{F}$ is the completion of a Galois extension of $F$, and such that $G(\breve{E})$ contains a $\mathrm{Gal}_{\breve{E} / F^{-}}$-stable Iwahori subgroup $I$

- a subset $\Sigma \subseteq \mathrm{Gal}_{\breve{E} / F}$ generating $\mathrm{Gal}_{\breve{E} / F}$

- a concave function $f: \Phi \cup\{0\} \rightarrow \tilde{\mathbb{R}}_{\geqslant 0} \backslash\{\infty\}$, such that $I^{f}$ is $\mathrm{Gal}_{\breve{E} / F^{-}}$stable

- a function $\underline{w}_{f}: \Sigma \rightarrow D_{G_{\breve{E}}, f}$

- an element $b \in G(\breve{E})$.

We define the extended affine Deligne-Lusztig set $X_{\underline{w}_{f}}^{f}(b)$ attached to $\left(G, E / F, f, \Sigma, \underline{w}_{f}, b\right)$ as the subset

$$
X_{\underline{w}_{f}}^{\Sigma, f}(b):=\left\{x \in \mathscr{F}^{f}(\bar{k}): \operatorname{inv}^{f}(x, b \gamma(x))=\underline{w}_{f}(\gamma) \forall \gamma \in \Sigma\right\} \subseteq \mathscr{F}^{f}(\bar{k}) .
$$

2.2.2. Left action by the $\mathrm{Gal}_{\breve{E} / F^{-}}$stabilizer of $b$. For $b \in G(\breve{E})$, let $J_{b}$ be the $\Sigma$-stabilizer of $b$, i.e., the algebraic group over $F$ defined by

$$
J_{b}(R):=\left\{g \in G\left(R \otimes_{F} \breve{E}\right): g^{-1} b \gamma(g)=b \forall \gamma \in \Sigma\right\}
$$

for any $F$-algebra $R$. Then $J_{b}(F)$ acts on $X_{\underline{w}_{f}}^{f}(b)$ by left multiplication for any $f$ and any $\underline{w}_{f}$. If $g \geqslant f$ and $\underline{w}_{g}$ lies over $\underline{w}_{f}$, then $X_{\underline{w}_{g}}^{g}(b)$ lies over $X_{\underline{w}_{f}}^{f}(b)$ and the $J_{b}(F)$-actions are compatible. 
2.2.3. Right action on $X_{\underline{w}_{f}}^{f}(b)$ by the stabilizer of $\underline{w}_{f}$. Let $w \in \tilde{W}$. If $I^{f}$ is normal in $I$, then $Z(\breve{E}) I / I^{f}$ acts on $D_{G_{\breve{E}}, f}$ by left and right multiplication and we obtain a (right) action of $Z(\breve{E}) I / I^{f}$ on the set of maps $\Sigma \rightarrow D_{G_{\breve{E}}, f}$ by $\left(\underline{w}_{f}, i\right) \mapsto \underline{w}_{f} . i$, where $\left.\underline{w}_{f} . i\right)(\gamma):=i^{-1} \underline{w}_{f}(\gamma) \gamma(i)$ for any $i \in Z(\breve{E}) I / I^{f}, \gamma \in \Sigma$. This inflates to an action of $Z(\breve{E}) I$ on the same set.

Lemma 2.2. Let $\left(G, E / F, \Sigma, f, \underline{w}_{f}, b\right)$ be as in Definition 2.1. Assume that $f \geqslant f_{I}$ and that $I^{f}$ is normal in $I$. For $i \in Z(\breve{E}) I$, the map $x I^{f} \mapsto x i I^{f}$ defines an isomorphism $X_{\underline{w}_{f}}^{f}(b) \stackrel{\sim}{\rightarrow} X_{\underline{w}_{f} . i}^{f}(b)$.

Proof. The proof is an easy computation.

Thus if $I^{f}$ is normal in $I$, the group

$$
\tilde{I}_{f, \underline{w}_{f}}:=\left\{i \in Z(\breve{E}) I: \underline{w}_{f} \cdot i=\underline{w}_{f}\right\}
$$

acts on $X_{\underline{w}_{f}}^{f}(b)$ by right multiplication. We have the subgroup $I_{f, \underline{w}_{f}}:=\tilde{I}_{f, \underline{w}_{f}} \cap I$. It is clear that $I_{f, \underline{w}_{f}} \supseteq I^{f}$ and that the right $\tilde{I}_{f, \underline{w}_{f}}$-action on $X_{\underline{w}_{f}}^{f}(b)$ factors through an action of $\tilde{I}_{f, \underline{w}_{f}} / I^{f}$.

Lemma 2.3. $X_{\underline{w}_{f}}^{f}(b)$ is a $I_{f, \underline{w}_{f}}$-torsor over the underlying Iwahori-level set (resp. variety, if a variety structure is provided) $X_{\underline{w}}(b)$.

Proof. This follows immediately from Lemma 2.2 .

2.2.4. Scheme structure. A priori it is not clear that $X_{\underline{w}_{f}}^{f}(b)$ can be equipped with a scheme structure in a natural way. In the following case this is possible.

Proposition 2.4. Let $\left(G, E / F, \Sigma, f, \underline{w}_{f}, b\right)$ be as in Definition 2.1 with $f \geqslant f_{I}$. Assume that $\Sigma$ is finite and contains a lift of a power of the Frobenius element in $\mathrm{Gal}_{\breve{F} / F}$ and that the action of $I^{f}$ on $I \underline{\dot{w}}_{f}(\gamma) I / I^{f}$ by left multiplication possesses a geometric quotient for any $\gamma \in \Sigma$, where $\underline{\dot{w}}_{f}(\gamma)$ is any preimage of $\underline{w}_{f}(\gamma)$ in $G(\breve{E})$. Then the subset $X_{\underline{w}_{f}}^{f}(b) \subseteq \mathscr{F} f$ is locally closed, and hence can be equipped with the induced reduced sub-Ind-scheme structure.

Proof. We write $X_{\underline{w}_{f}}^{\Sigma, f}(b)$ for $X_{\underline{w}_{f}}^{f}(b)$. Let $\underline{w}$ be the composition of $\underline{w}_{f}$ with the projection $D_{G_{\breve{E}}, f} \rightarrow \tilde{W}$. If $\tilde{\sigma}$ is a lift in $\operatorname{Gal}(\breve{E} / F)$ of a power of the Frobenius in $\mathrm{Gal}_{\breve{F} / F}$, then $\mid \mathrm{HV}$ Corollary 6.5 shows that $X_{\underline{w}(\tilde{\sigma})}^{\{\tilde{\sigma}\}, f_{I}}(b)$ is a locally closed subset of $\mathscr{F}$. Moreover, it is a scheme locally of finite type over a finite extension of $k_{E}$. Now, $X_{\underline{w}}^{\Sigma, f_{I}}(b)$ is the subset of $X_{\underline{w}(\tilde{\sigma})}^{\{\tilde{\sigma}\}, f_{I}}(b)$ cut out by the finitely many locally closed conditions $x^{-1} b \gamma(x) \in \underline{w}(\gamma)$. This shows that $X_{\underline{w}}^{\Sigma, f_{I}}(b)$ is locally closed and locally of finite type over $k_{E}$.

Consider the preimage $\tilde{X}$ of $X_{w}^{\Sigma, f_{I}}(b)$ under $\mathscr{F}^{m} \rightarrow \mathscr{F}$. By $[\mathrm{PR}$ Theorem 1.4, the projection $\beta: L G \rightarrow \mathscr{F}^{m}$ admits sections locally for the étale topology. Let $U \rightarrow \tilde{X}$ be étale, such that there is a section $s: U \rightarrow \beta^{-1}(U)$ of $\beta$. Consider the composition of the two morphisms

$$
\psi: U \rightarrow \prod_{\gamma \in \Sigma} \beta^{-1}(U) \times U \rightarrow \prod_{\gamma \in \Sigma} \mathscr{F}^{f},
$$

where the first is given by $x \mapsto\left(s(x)^{-1}, b \gamma(x)\right)_{\gamma \in \Sigma}$ and the second is just the componentwise restriction of the left multiplication action of $G(\breve{E})$ on $\mathscr{F}^{f}$. As $U$ lies over $\tilde{X}$, this composed morphism factors through the inclusion $\prod_{\gamma \in \Sigma} I \underline{\dot{w}}_{f}(\gamma) I / I^{f} \subseteq \prod_{\gamma \in \Sigma} \mathscr{F} f$. Let $\pi_{\gamma}: I \underline{\dot{w}}_{f}(\gamma) I / I^{f} \rightarrow$ $D_{G_{\breve{E}}, f}(\underline{w}(\gamma))$ denote the geometric quotient with respect to the left multiplication action by $I^{f}$. Let $\pi=\prod_{\gamma \in \Sigma} \pi_{\gamma}$. Then the composition

$$
U \stackrel{\psi}{\rightarrow} \prod_{\gamma \in \Sigma} I w I / I^{f} \stackrel{\pi}{\rightarrow} \prod_{\gamma \in \Sigma} D_{G_{\breve{E}}, f}(\underline{w}(\gamma)) .
$$


is independent of the choice of the section $s$. Moreover, it sends a $\bar{k}$-point $x$ to the tuple $\left(I^{f} x^{-1} b \gamma(x) I^{f}\right)_{\gamma \in \Sigma}$. Thus, étale locally, $X_{\underline{w}_{f}}^{\Sigma, f}(b)$ is just the preimage of a $\bar{k}$-point under the composite morphism (2.1). This finishes the proof.

The condition about the existence of geometric quotients is satisfied in many cases. This will be studied in detail in a future work.

\section{3. $\mathrm{GL}_{2}$, TAMELY RAMified CASE: GeOMEtRY}

From here and until the end of the article we set $G=\mathrm{GL}_{2}$ and assume that char $k \neq 2$. After fixing some notation in Section 3.1. we study some extended affine Deligne-Lusztig varieties of Iwahori level in Section 3.2 and of higher levels in Section 3.3 .

\subsection{Some preliminaries in the $\mathrm{GL}_{2}$-case.}

3.1.1. Basic notation. Let $\breve{F} / F=k((t)), k$ be as in Section 2.1.1 with char $k \neq 2$. Let $E / F$ be a tamely ramified degree 2 extension and let $\breve{E}:=E \breve{F}$. We can find an uniformizer $u \in E$ such that $u^{2}=t$. Then $E=k((u)), \breve{E}=\bar{k}((u))$. For an algebraic extension $M$ of $F$, we denote by $\mathcal{O}_{M}$ resp. $\mathfrak{p}_{M}$ its ring of integers resp. its maximal ideal. We have $\mathcal{O}_{E}=k \llbracket u \rrbracket$, $\mathcal{O}_{\breve{E}}=\bar{k} \llbracket u \rrbracket$. The Galois group of $\breve{E} / F$ is generated by the two commuting elements $\sigma, \tau$ given by $\sigma\left(\sum_{i} a_{i} u^{i}\right)=\sum_{i} a_{i}^{q} u_{i}$ and $\tau\left(\sum_{i} a_{i} u^{i}\right)=\sum_{i}(-1)^{i} a_{i} u^{i}$. We set $\Sigma:=\{\sigma, \tau\}$,

3.1.2. Level subgroups. We use the standard Iwahori subgroup $I \subseteq G(\breve{E})$ and the filtration of it given for $m \geqslant 0$ by

$$
I^{m}:=\left(\begin{array}{cc}
1+\mathfrak{p}_{\breve{E}}^{m+1} & \mathfrak{p}_{\breve{E}}^{m} \\
\mathfrak{p}_{\breve{E}}^{m+1} & 1+\mathfrak{p}_{\breve{E}}^{m+1}
\end{array}\right) \subseteq I:=\left(\begin{array}{cc}
\mathcal{O}_{\breve{E}}^{\times} & \mathcal{O}_{\breve{E}} \\
\mathfrak{p}_{\breve{E}} & \mathcal{O}_{\breve{E}}^{\times}
\end{array}\right) .
$$

We write $\mathscr{F}$ for the affine flag manifold of $G_{\breve{E}}$ and $\mathscr{F}^{m}$ for its cover corresponding to $I^{m}$ (see Section 2.1.8.

3.1.3. Subgroups of $G(F)$. Consider the $\mathcal{O}_{F}$-subalgebra

$$
\mathfrak{J}:=\left(\begin{array}{cc}
\mathcal{O}_{F} & \mathcal{O}_{F} \\
\mathfrak{p}_{F} & \mathcal{O}_{F}
\end{array}\right)
$$

of $M_{2}\left(\mathcal{O}_{F}\right)$. Then the units $U_{\mathfrak{J}}$ of $\mathfrak{J}$ form a compact subgroup of $G(F)$. Note that $U_{\mathfrak{J}}=I^{\left\langle\mathrm{Gal}_{\breve{E} / F}\right\rangle}$. Further, we fix the embedding of $F$-algebras

$$
\iota: E \hookrightarrow M_{2}(F), \quad \iota(u)=\varpi:=\left(\begin{array}{c}
1 \\
t
\end{array}\right)
$$

(here and further, omitted entries are zeros). Via $\iota$ we consider $E^{\times}$as a subgroup of $G(F)$. The center of $G(F)$ is $\iota\left(F^{\times}\right)$. Usually we omit $\iota$ from the notation and write $E^{\times} \subseteq G(F)$, etc. We have $U_{E}=U_{\mathfrak{J}} \cap E^{\times}$.

3.1.4. Root subgroups. The extended set of roots $\Phi \cup\{0\}$ consists of three elements. Denote by + resp. - the positive resp. the negative root. For $* \in \Phi \cup\{0\}$, we denote by

$$
e_{*}: U_{*} \rightarrow G
$$

the embedding of the root subgroup. Thus, for $a \in \breve{E}, e_{+}(a)=\left(\begin{array}{cc}1 & a \\ & 1\end{array}\right), e_{0}(c, d)=\left(\begin{array}{cc}c & \\ & d\end{array}\right)$, etc.

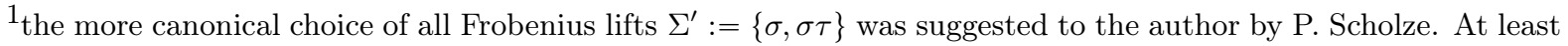
in the cases we study in this article, this choice will lead to the same results as $\Sigma$ from the text.
} 
3.1.5. Slices of positive loops. Consider the additive group $\mathbb{G}_{a}$ over $\breve{E}$. The group $\mathbb{G}_{a}(\breve{E})$ has a filtration by subgroups $\mathbb{G}_{a}(\breve{E})_{\lambda}:=u^{\lambda} \bar{k} \llbracket u \rrbracket$ for $\lambda \in \mathbb{Z}_{\geqslant 0}$. There is a unique smooth model $\mathbb{G}_{a, \lambda}$ of $\mathbb{G}_{a}$ over $\mathcal{O}_{\breve{E}}$, such that $\mathbb{G}_{a, \lambda}\left(\mathcal{O}_{\breve{E}}\right)=\mathbb{G}_{a}(\breve{E})_{\lambda}$. For any $\mu \leqslant \lambda$, there exists a unique morphism $\mathbb{G}_{a, \lambda} \rightarrow \mathbb{G}_{a, \mu}$, inducing the natural embedding $u^{\lambda} k \llbracket u \rrbracket \hookrightarrow u^{\mu} k \llbracket u \rrbracket$ (see $\mid \mathrm{BT} 2$. Section 1.7). Let $L^{+}$denote the positive loop group functor from $\bar{k} \llbracket u \rrbracket$-schemes to $\bar{k}$-schemes. For non-negative integers $\mu \leqslant \lambda$, we define

$$
L_{[\mu, \lambda]} \mathbb{G}_{a}:=L^{+} \mathbb{G}_{a, \mu} / L^{+} \mathbb{G}_{a, \lambda+1} .
$$

This is a smooth $\bar{k}$-group of finite type and we have canonically $L_{[\mu, \lambda]} \mathbb{G}_{a}(\bar{k})=u^{\mu} \bar{k}[u] / u^{\lambda+1} \bar{k}[u]$. Replacing $\mathbb{G}_{a}$ by $\mathbb{G}_{m}$ and using the filtration on $\mathbb{G}_{m}(\breve{E})=\bar{k}((u))^{\times}$given by $\mathbb{G}_{m}(\breve{E})_{0}=\bar{k} \llbracket u \rrbracket^{\times}$, $\mathbb{G}_{m}(\breve{E})_{\lambda}=1+u^{\lambda} \bar{k} \llbracket u \rrbracket$ for $\lambda>0$, we obtain in exactly the same way the $\bar{k}$-groups $L_{[\mu, \lambda]} \mathbb{G}_{m}$. All these groups uniquely descend to smooth group schemes over $k$.

Let now $0 \leqslant \mu \leqslant \lambda \leqslant \lambda^{\prime}$. We have the natural projection, which comes from reduction $\bmod u^{\lambda+1}$ :

$$
p_{\lambda, \lambda^{\prime}}: L_{\left[\mu, \lambda^{\prime}\right]} \mathbb{G}_{a} \rightarrow L_{[\mu, \lambda]} \mathbb{G}_{a}
$$

Moreover, there are group-theoretic sections

$$
s_{\lambda, \lambda^{\prime}}: L_{[\mu, \lambda]} \mathbb{G}_{a} \rightarrow L_{\left[\mu, \lambda^{\prime}\right]} \mathbb{G}_{a}
$$

of $p_{\lambda, \lambda^{\prime}}$, sending $\sum_{i=0}^{\lambda} a_{i} u^{i}$ to $\sum_{i=0}^{\lambda} a_{i} u^{i}+\sum_{i=\lambda+1}^{\lambda^{\prime}} 0 u^{i}$. Then the image of $s_{\lambda, \lambda^{\prime}}$ is a closed subgroup scheme of $L_{\left[\mu, \lambda^{\prime}\right]} \mathbb{G}_{a}$ and we denote it by $L_{\left[\mu, \lambda^{\prime}\right]}^{\leqslant \lambda} \mathbb{G}_{a}$. For $a \in L_{\left[\mu, \lambda^{\prime}\right]} \mathbb{G}_{a}$, we use the shortcut notation $\left.a\right|_{\lambda}:=s_{\lambda, \lambda^{\prime}}\left(p_{\lambda, \lambda^{\prime}}(a)\right)$.

3.1.6. Schubert cells. Let $\tilde{W}$ denote the extended affine Weyl group of $G_{\breve{E}}$ relative to the diagonal torus (as in Section 2.1.10). Let $v \in \tilde{W}$ and let $\dot{v} \in G(\breve{E})$ be a lift. We denote by $C_{v}=I \dot{v} I / I \subseteq \mathscr{F}$ the open Schubert cell attached to $v$. There is a parametrization (depending of $\dot{v}$ ) of $C_{v}$ given by:

$$
\psi_{\dot{v}}: L_{[\mu, \mu+\ell(v)-1]} \mathbb{G}_{a} \stackrel{\sim}{\rightarrow} C_{v}, \quad a \mapsto e_{ \pm}(a) \dot{v} I
$$

where $\mu \in\{0,1\}$ and the sign in $e_{ \pm}$depend on $v$, and $\ell(v)$ is the length of $v$. E.g., for $\dot{v}=$ $\left(\begin{array}{ll}u^{k} & u^{-k}\end{array}\right)$ resp. $\dot{v}=\left(\begin{array}{cc}u^{k+1} & u^{-k}\end{array}\right)$, this parametrization is given by:

$$
\psi_{i}: L_{[1, \ell(v)]} \mathbb{G}_{a} \stackrel{\sim}{\rightarrow} C_{v}, \quad a \mapsto e_{-}(a) \dot{v} I,
$$

where $a=\sum_{i=1}^{\ell(v)} a_{i} u^{i}$ (note that $\ell(v)=2 k-1$ resp. $\ell(v)=2 k$ ).

3.1.7. Schubert cells in higher levels. For $m \geqslant 0$, let $\mathrm{pr}_{m}: \mathscr{F}^{m} \rightarrow \mathscr{F}$ be the natural projection. Let $v \in \tilde{W}$ with lift $\dot{v}$ to $G(\breve{E})$. Let $C_{v}^{m}:=\operatorname{pr}_{m}^{-1}\left(C_{v}\right)$. We give a parametrization of $C_{v}^{m}$ for $v, \dot{v}$ as in (3.1) (for other $v \in \tilde{W}$ the parametrization is defined similarly). There is a well-defined injective morphism $L_{[1, \ell(v)+m]} \mathbb{G}_{a} \rightarrow C_{v}^{m}$ given by $a \mapsto e_{-}(a) \dot{v} I$. Using it we get a diagram

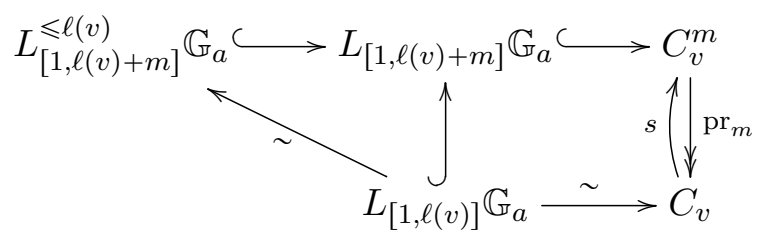

where the lower horizontal map is $\psi_{\dot{v}}$, the left vertical map is $s_{\ell(v), \ell(v)+m}$, and the section $s$ to $\operatorname{pr}_{m}$ is defined such that the diagram commutes. As $C_{v}^{m} \rightarrow C_{v}$ is a $I / I^{m}$-torsor, $s$ induces the 
trivialization isomorphism $C_{v} \times I / I^{m} \stackrel{\sim}{\rightarrow} C_{v}^{m}$ given by $x, i \mapsto s(x) i$. Using a parametrization of $I / I^{m}$, we obtain the following explicit parametrization of $C_{v}^{m}$ (depending on $\dot{v}$ ):

$\psi_{\dot{v}}^{m}: L_{[1, \ell(v)+m]}^{\leqslant \ell(v)} \mathbb{G}_{a} \times L_{[0, m]} \mathbb{G}_{m}^{2} \times L_{[0, m-1]} \mathbb{G}_{a} \times L_{[1, m]} \mathbb{G}_{a} \stackrel{\sim}{\longrightarrow} C_{v}^{m}=I \dot{v} I / I^{m}$

$$
a, C, D, A, B \quad \mapsto \quad e_{-}(a) \dot{v} e_{0}(C, D) e_{+}(A) e_{-}(B) I^{m} .
$$

3.1.8. Spaces of double cosets. Let $m \geqslant 0$ be an integer and let $w \in \tilde{W}$. It can be seen that $C_{w}^{m}$ possesses a geometric quotient for the $I^{m}$-action by left multiplication. The set of its $\bar{k}$-valued points is the set of double cosets $D_{G_{\breve{E}}, m}(w)=I^{m} \backslash I w I / I^{m}$. Let $\dot{w}=\left(\begin{array}{cc}u^{n} & u^{-n}\end{array}\right)$ with $n>0$. Let $w$ be the image of $\dot{w}$ in $\tilde{W}$. An explicit parametrization of $D_{G_{\breve{E}}, m}(w)$ is given by

$$
\begin{aligned}
\phi_{\dot{w}}^{m}: L_{[0, m]} \mathbb{G}_{m}^{2} \times L_{[1, m]} \mathbb{G}_{a}^{2} & \stackrel{\sim}{\longrightarrow} D_{G_{\breve{E}}, m}(w)=I^{m} \backslash I w I / I^{m} \\
(C, D),(E, B) & \mapsto I^{m} e_{-}(E) \dot{w} e_{0}(C, D) e_{-}(B) I^{m} .
\end{aligned}
$$

3.1.9. Bruhat-Tits buildings. (cf. Section 2.1.3) For any finite extension $M$ of $F$ or $\breve{F}$, the Bruhat-Tits building $\mathscr{B}_{M}$ of $G$ over $M$ is an one-dimensional simplicial complex and it carries a $\mathrm{Gal}_{M / F^{-a c t i o n}}$ if $M / F$ is Galois. We identify the subcomplex $\mathscr{B}_{\breve{E}}^{\langle\sigma\rangle}$ of $\mathscr{B}_{\breve{E}}$ with $\mathscr{B}_{E}$. Moreover, as $\breve{E} / \breve{F}$ is tamely ramified, the embedding $\mathscr{B}_{\breve{F}} \hookrightarrow \mathscr{B}_{\breve{E}}$ identifies $\mathscr{B}_{\breve{F}}$ with $\mathscr{B}_{\breve{E}}^{\langle\tau\rangle}$ as subsets. The simplicial complex $\mathscr{B}_{\breve{E}}^{\langle\tau}$ is obtained from $\mathscr{B}_{\breve{F}}$ by adding an extra vertex in the middle of each alcove. Thus any alcove of $\mathscr{B}_{\breve{F}}$ 'contains' two alcoves of $\mathscr{B}_{\breve{E}}^{\langle\tau}$. Any vertex of $\mathscr{B}_{\breve{F}}$ has an associated type in $\mathbb{Z} / 2 \mathbb{Z}=\{0,1\}$, which is defined as the $t$-valuation modulo 2 of the determinant of the lattice, representing it. Similarly, we attach to any vertex of $\mathscr{B}_{\breve{E}}$ its relative type in $\frac{1}{2} \mathbb{Z} / \mathbb{Z}=v_{t}\left(\breve{E}^{\times}\right) / v_{t}\left(\breve{F}^{\times}\right)$, defined as the class modulo $\mathbb{Z}$ of the $t$-valuation of the determinant of the representing lattice. The same considerations also apply to the relationship between the $\sigma$-stable subcomplexes $\mathscr{B}_{F} \leadsto \mathscr{B}_{E}^{\langle\tau\rangle} \subseteq \mathscr{B}_{E}$.

3.1.10. Vertex of departure. In the proofs below we have to use the simple combinatorics of the tree $\mathscr{B}_{\breve{E}}$. Therefore, following $[\overline{R e}]$ we introduce the notion of the vertex of departure. Let $\mathscr{C} \subseteq \mathscr{B}_{\breve{E}}$ be a connected non-empty subcomplex. For any alcove $C$ of $\mathscr{B}_{\breve{E}}$, which is not contained in $\mathscr{C}$, there is a unique gallery $\Gamma=\left(C_{0}, C_{1}, \ldots, C_{d}\right)$ of minimal length $d$, such that $C_{0}=C$ and $C_{d}$ is not contained in $\mathscr{C}$ and has a (unique) vertex which is contained in $\mathscr{C}$. This vertex of $C_{d}$ is called the vertex of departure of $C$ from $\mathscr{C}$. The same considerations can also be applied to $\mathscr{B}_{E}$ and a connected subcomplex.

3.1.11. Connected components of $\mathscr{F}$. It is well-known ( $[\mathrm{PR}]$ Theorem 5.1) that $v_{u} \circ \operatorname{det}$ induces an isomorphism

$$
v_{u} \circ \operatorname{det}: \pi_{0}(\mathscr{F}) \stackrel{\sim}{\rightarrow} \mathbb{Z} .
$$

Denote the connected component of $\mathscr{F}$ corresponding to the integer $i$ by $\mathscr{F}^{(i)}$. Note that the alcoves of $\mathscr{B}_{\breve{E}}$ can be identified with $\bar{k}$-points of $\mathscr{F}^{(0)}$ and that there are (non-canonical) isomorphisms $\mathscr{F}^{(0)} \stackrel{\sim}{\rightarrow} \mathscr{F}^{(i)}$. Further, denote by $\mathscr{F} \equiv i$ the preimage under $v_{u}$ odet in $\mathscr{F}$ of $2 \mathbb{Z}+i$. This divides $\mathscr{F}$ in two disjoint sub-Ind-schemes $\mathscr{F}=\mathscr{F} \equiv 0 \dot{\cup} \mathscr{F} \equiv 1$. Note that the subgroup $G(F)$ of $G(\breve{E})$ acts transitively (by left multiplication) on the set of connected components of $\mathscr{F} \equiv i$ and that the action of $\left(\begin{array}{c} \\ u\end{array}\right) \in G(\breve{E})$ interchanges the two parts $\mathscr{F} \equiv 0$ and $\mathscr{F} \equiv 1$ of $\mathscr{F}$. 
3.1.12. Commutation relations. We will need the following relations: for $a, b \in \breve{E}$ with $N:=$ $1+a b \neq 0$, we have

$$
\begin{aligned}
& e_{+}(a) e_{-}(b)=e_{-}\left(b N^{-1}\right) e_{+}(a N) e_{0}\left(N, N^{-1}\right) \\
& e_{-}(b) e_{+}(a)=e_{0}\left(N^{-1}, N\right) e_{+}(a N) e_{-}\left(b N^{-1}\right) .
\end{aligned}
$$

\subsection{Structure of $X_{\underline{w}}(1)$ at the Iwahori-level in some cases.}

We write $X_{\underline{w}}(1)$ instead of $X_{\underline{w}}^{f_{I}}(1)$.

Notation 3.1. Let $w:=\left(\begin{array}{cc}u^{n} & u^{-n}\end{array}\right) \in \tilde{W}$ with some integer $n>0$ and let $\underline{w}: \Sigma \rightarrow \tilde{W}$ be defined by $\underline{w}(\sigma)=1$ and $\underline{w}(\tau)=w$. Further, we set

$$
\dot{v}=\dot{v}(w):= \begin{cases}\left(\begin{array}{c}
u^{-k} \\
u^{k}
\end{array}\right) & \text { if } n=2 k-1>0 \text { is odd }, \\
\left(\begin{array}{cc}
u^{k+1} & u^{-k}
\end{array}\right) & \text { if } n=2 k>0 \text { is even. }\end{cases}
$$

In both cases let $v$ be the image of $\dot{v}$ in $\tilde{W}$ and let $D_{w}^{\tau}$ be the set of $k$-rational points of $C_{v}$ lying in the locus $a_{1} \neq 0$ with respect to the coordinates (3.1). In particular, $D_{w}^{\tau}$ is just a finite discrete union of $k$-rational points.

It will follow from the proof of Proposition 3.3 (or can be seen directly) that $D_{w}^{\tau}$ is stable under the left multiplication action by $U_{\mathfrak{J}}$ on $\mathscr{F}$.

Remark 3.2. If in Notation 3.1, $n$ is odd, then $C_{v}$ is contained in the connected component $\mathscr{F}^{(0)}$ of $\mathscr{F}$, i.e., its points can be seen as alcoves in $\mathscr{B}_{\breve{E}}$. Moreover, they all are $k$-rational, hence lie in $\mathscr{B}_{E}$. Let $P_{1 / 2}$ be the vertex of the base alcove (= the alcove corresponding to $I$ ) of $\mathscr{B}_{E}$ with relative type $\frac{1}{2}$ (see Section 3.1 .9 . Then $D_{w}^{\tau}$ corresponds to the set of the alcoves contained in $\mathscr{B}_{E}$, having relative position $v$ to the base alcove and having $P_{1 / 2}$ as the vertex of departure from $\mathscr{B}_{E}^{\langle\tau\rangle}$.

Proposition 3.3. Let $\underline{w}$ be as in Notation 3.1. There is an isomorphism

$$
X_{\underline{w}}(1) \cong \coprod_{g \in G(F) / U_{\mathfrak{J}}} g D_{v}^{\tau}
$$

equivariant for the left $G(F)$-action. In particular, $X_{\underline{w}}(1)$ is a zero-dimensional reduced $k$ variety, containing only $k$-rational points.

Proof. Let first $n$ be odd. The natural action of $G(F)$ on $X_{\underline{w}}(1)$ induces a transitive action of $G(F)$ on the set

$$
\left\{X_{\underline{w}}(1) \cap \mathscr{F}^{(2 i)}: i \in \mathbb{Z}\right\}
$$

of subsets of $X_{\underline{w}}(1)$. This follows by taking any element $g \in G(F)$ with $v_{u}(\operatorname{det}(g))=2$. The stabilizer of $\mathscr{F}^{(\overline{0})}$ in $G(F)$ is

We deduce

$$
H:=\left(v_{u} \circ \operatorname{det}\right)^{-1}(0) \subseteq G(F) .
$$

$$
X_{\underline{w}}(1) \cap \mathscr{F}^{\equiv 0}=\coprod_{g \in G(F) / H} g \cdot\left(X_{\underline{w}}(1) \cap \mathscr{F}^{(0)}\right) .
$$


The $\bar{k}$-rational points of $X_{\underline{w}}(1) \cap \mathscr{F}^{(0)}$ can be identified with alcoves in $\mathscr{B}_{\breve{E}}$, which satisfy two conditions (defined by $\underline{w}(\sigma)=1$ and $\underline{w}(\tau)=w$ ) on the relative position with respect to their $\sigma$-resp. $\tau$-translate. The $\sigma$-condition simply assures that each of the alcoves contained in $X_{\underline{w}}(1)$ is $\sigma$-stable, i.e., is contained in $\mathscr{B}_{E}$. Let $\left(\mathscr{B}_{E}^{\langle\tau\rangle}\right)^{(1 / 2)}$ be the set of all vertices of $\mathscr{B}_{E}^{\langle\tau\rangle}$ of relative type $\frac{1}{2}$. For an alcove $C$ of $\mathscr{B}_{E}$, which is not contained in $\mathscr{B}_{E}^{\langle\tau\rangle}$, let $\Gamma_{C, \tau}$ denote the unique minimal gallery connecting $C$ with $\mathscr{B}_{E}^{\langle\tau\rangle}$. Taking into account the types of the involved vertices, we deduce (exactly as in Iv1]) that

$$
X_{\underline{w}}(1) \cap \mathscr{F}^{(0)}=\coprod_{P \in\left(\mathscr{B}_{E}^{\langle\tau\rangle}\right)^{(1 / 2)}}\left\{C: \begin{array}{c}
C \text { is an alcove in } \mathscr{B}_{E} \text { with vertex of departure from } \\
\mathscr{B}_{E}^{\langle\tau\rangle} \text { equal to } P \text { and length of } \Gamma_{C, \tau} \text { equal to } n-1
\end{array}\right\},
$$

with $n$ as in Notation 3.1. Let $P_{1 / 2}$ be the vertex of type $\frac{1}{2}$ of the base alcove of $\mathscr{B}_{E}$. Observe that for $P=P_{1 / 2}$, the set of alcoves $C$ on the right hand side of (3.6) is simply $D_{w}^{\tau}$ (cf. Remark 3.2 .

Now, $\left(\mathscr{B}_{E}^{\langle\tau\rangle}\right)^{(1 / 2)}$ can be canonically identified with the set of alcoves in $\mathscr{B}_{F}$ (see Section 3.1.9). The natural action of $H$ on $\mathscr{B}_{F}$ induces a transitive action of $H$ on the set of alcoves of $\mathscr{B}_{F}$, and the stabilizer of the base alcove in $\mathscr{B}_{F}$ is precisely $U_{\mathfrak{J}} \subseteq H$. Combining these observations, we obtain a natural $H$-equivariant bijection

$$
H / U_{\mathfrak{J}} \cong\left(\mathscr{B}_{E}^{\langle\tau\rangle}\right)^{(1 / 2)}, \quad h U_{\mathfrak{J}} \mapsto h P_{1 / 2} .
$$

Combining (3.5), (3.6) and (3.7), we deduce

$$
X_{\underline{w}}(1) \cap \mathscr{F} \equiv 0=\coprod_{g \in G(F) / H} g \cdot\left(X_{\underline{w}}(1) \cap \mathscr{F}^{(0)}\right)=\coprod_{g \in G(F) / H} g \cdot\left(\coprod_{h \in H / U_{\mathfrak{J}}} h D_{w}^{\tau}\right)=\coprod_{g \in G(F) / U_{\mathfrak{J}}} g D_{w}^{\tau} .
$$

It remains to show that $X_{\underline{w}}(1) \cap \mathscr{F} \equiv 1=\varnothing$. This can be done as follows: let $h=\left(\begin{array}{c}1 \\ u\end{array}\right)$. By Lemma 2.2, the map $x I \mapsto x h I$ defines an isomorphism

$$
X_{\underline{y}}(1) \cap \mathscr{F} \equiv 1 \stackrel{\sim}{\rightarrow} X_{\underline{y} . h}(1) \cap \mathscr{F} \equiv 0
$$

for any $\underline{y}: \Sigma \rightarrow \tilde{W}$, where $(\underline{y} . h)(\gamma):=h^{-1} \underline{y}(\gamma) \gamma(h)$ for $\gamma \in \Sigma$. Thus it is enough to show that $X_{\underline{w} . h}(1) \cap \mathscr{F} \equiv 0=\varnothing$, where $(\underline{w} \cdot h)(\sigma)=1,(\underline{w} \cdot h)(\tau)=h^{-1} w \tau(h)=\left(\begin{array}{cc}u^{n-1} \\ u^{1-n}\end{array}\right) \in \tilde{W}$. This follows from (3.5) and $X_{\underline{w} . h}(1) \cap \mathscr{F}^{(0)}=\varnothing$. This last follows from the combinatorics of $\mathscr{B}_{E}$ as $n-1$ is even: one has to use the fact that a vertex $P$ of $\mathscr{B}_{E}$ of relative type 0 cannot be the vertex of departure from $\mathscr{B}_{E}^{\langle\tau\rangle}$ for a non- $\tau$-stable alcove $C$ of $\mathscr{B}_{E}$, as all alcoves having $P$ as a vertex lie in $\mathscr{B}_{E}^{\langle\tau\rangle}$.

Let now $n$ be even. Applying the isomorphism 3.8 with $h$ replaced by $h^{-1}$, we reduce to determining $X_{\underline{y}}(1)$ with $\underline{y}(\sigma)=1$ and $\underline{y}(\tau)=\left(\begin{array}{c}u^{n-1} \\ u^{1-n}\end{array}\right)$, where we can proceed exactly as in the case $n$ odd (after replacing $n$ by $-n$ ).

\subsection{Structure of $X_{\underline{w}_{m}}^{m}(1)$ in some cases.}

Continuing with notations from preceding sections, we now study higher level covers of $X_{\underline{w}}(1)$. For $x \in G(\breve{E})$ we denote the image of $x$ in $D_{G_{\breve{E}}, m}$ again by $x$, if no ambiguity can occur. 
Notation 3.4. Let $n, w, \underline{w}$ be as in Notation 3.1. We define the lift $\dot{w} \in G(\breve{E})$ of $w$ by

$$
\dot{w}:= \begin{cases}\left(\begin{array}{c}
(-1)^{k} u^{1-2 k} \\
(-1)^{k+1} u^{2 k-1}
\end{array}\right) & \text { if } n=2 k-1>0 \text { is odd, } \\
\left(\begin{array}{cc}
(-1)^{k} u^{2 k} u^{-2 k} \\
(-1)
\end{array}\right. & \text { if } n=2 k>0 \text { is even. }\end{cases}
$$

Moreover, let $m \geqslant 1$ be an odd integer. Let $\underline{w}_{m}: \Sigma \rightarrow D_{G_{\breve{E}}, m}$ be the lift of $\underline{w}$ defined by

$$
\underline{w}_{m}(\sigma):=1, \quad \underline{w}_{m}(\tau):=\dot{w} .
$$

We have $J_{1}(F)=G(F)$ and hence by Section 2.2 we obtain the group actions

$$
G(F) \subset X_{\underline{w}_{m}}^{m}(1) り \tilde{I}_{m, \underline{w}_{m}} / I^{m} .
$$

Lemma 3.5. Let $m \geqslant 1$ be an odd integer. There is an isomorphism

$$
\tilde{I}_{m, \underline{w}_{m}} / I^{m} \stackrel{\sim}{\rightarrow}\left\{\left(\begin{array}{cc}
i_{1} & i_{2} \\
0 & \tau\left(i_{1}\right)
\end{array}\right): i_{1} \in E^{\times} / U_{E}^{m+1}, i_{2} \in E / \mathfrak{p}_{E}^{m}, v_{u}\left(i_{2}\right) \geqslant v_{u}\left(i_{1}\right)\right\} \subset Z(\breve{E}) I / I^{m} .
$$

In particular, there is a surjection induced by the projection onto the diagonal part

$$
\tilde{I}_{m, \underline{w}_{m}} / I^{m} \rightarrow E^{\times} / U_{E}^{m+1},
$$

under which $I_{m, \underline{w}_{m}} / I^{m}$ maps onto $U_{E} / U_{E}^{m+1}$.

Proof. The proof is an easy computation.

Recall from Section 3.1 .3 that we see $E^{\times}$as a subgroup of $G(F)$. This defines a left multiplication action of $E^{\times}$on $X_{\underline{w}_{m}}^{m}$ (1) (do not confuse this $E^{\times}$with the quotient $E^{\times}$of $\tilde{I}_{m, \underline{w}_{m}}$ acting on the right).

Definition 3.6. With notation from Notations 3.1 3.4, we define the discrete subscheme $Y_{\dot{w}}^{m}$ of $C_{v}^{m} \subseteq \mathscr{F}^{m}$ as follows. Let $a=\sum_{i=1}^{\ell(v)} a_{i} u^{i} \in L_{[1, \ell(v)+m]}^{<\ell(v)} \mathbb{G}_{a}(\bar{k})$ be as used in the parametrization (3.2) of $C_{v}^{m}$. Put $R:=u^{-1}(\tau(a)-a) \bmod u^{m+1}$. We define $Y_{\dot{w}}^{m}$ to be the subscheme of $C_{v}^{m}$ defined in coordinates $\psi_{\dot{v}}^{m}$ from $(3.2)$ by the following conditions:

$$
\begin{aligned}
& a, A, C \text { are } k \text {-rational } \\
& a_{1} \neq 0 \quad \text { in particular, } R \text { is invertible) } \\
& B=C \tau(C)^{-1} u^{n} \\
& D=R^{-1} \tau(C)\left(1+C \tau(C)^{-1} A u^{n}-C^{-1} \tau(C) \tau(A) u^{n}\right)
\end{aligned}
$$

(both last equations take place in $k[u] /\left(u^{m+1}\right)$ ). In particular, $Y_{\dot{w}}^{m}$ is just a finite discrete union of $k$-rational points. Moreover, let $y_{i}:=e_{0}\left(u^{i},(-u)^{i}\right)$ and define $\tilde{Y}_{\dot{w}}^{m} \subseteq \mathscr{F}^{m}$ to be (disjoint) union

$$
\tilde{Y}_{\dot{w}}^{m}:=\coprod_{i \in \mathbb{Z}} Y_{\dot{w}}^{m} \cdot y_{i} .
$$

It will follow from the proof of Theorem 3.8 that the right multiplication action of $I / I^{m}$ on $C_{v}^{m}$ restricts to an action of $I_{m, \underline{w}_{m}} / I^{m}$ on $Y_{\dot{w}}^{m}$, which in turn extends to a right $\tilde{I}_{m, \underline{w}_{m}} / I^{m}$-action on $\tilde{Y}_{\dot{w}}^{m}$.

Remark 3.7. The varieties $Y_{\dot{w}}^{m}, \tilde{Y}_{\dot{w}}^{m}$ depend on $\dot{w}$, not only on $w$, but the choice of the lift $\dot{w}$ of $w$ is not essential: another choices would give either empty varieties or varieties isomorphic to 
those attached to $\dot{w}$. The full study of these choices is not relevant for the goals of this article, so we restrict our attention to our choice $\dot{w}$.

Theorem 3.8. Let $m \geqslant 1$ be an odd integer. With notation as in Definition 3.6 assume that $m \leqslant \ell(w)=2 n-1$. Then $\tilde{Y}_{\dot{w}}^{m}$ (resp. $\left.Y_{\dot{w}}^{m}\right)$ is invariant under the left $E^{\times} U_{\mathfrak{J}^{-}}$(resp. $U_{\mathfrak{J}^{-}}$)action and the right $\tilde{I}_{m, \underline{w}_{m}} / I^{m}$ - (resp. $I_{m, \underline{w}_{m}} / I^{m}$-) action and there is an isomorphism

$$
X_{\underline{w}_{m}}^{m}(1) \cong \coprod_{g \in G(F) / E^{\times} U_{\mathfrak{J}}} g \tilde{Y}_{\dot{w}}^{m}
$$

equivariant for the left $G(F)$ - and right $\tilde{I}_{m, \underline{w}_{m}} / I^{m}$-actions. In particular, $X_{\underline{w}_{m}}^{m}$ (1) is a zerodimensional reduced $k$-variety, containing only $k$-rational points.

Proof. We claim that $X_{\underline{w}_{m}}^{m}(1) \cong \coprod_{g \in G(F) / U_{\mathfrak{J}}} g Y_{\dot{w}}^{m}$. As the natural projection $\mathscr{F} m \rightarrow \mathscr{F}$ restricts to a $G(F)$-equivariant projection $\mathrm{p}_{m}: X_{\underline{w}_{m}}^{m}(1) \rightarrow X_{\underline{w}}(1)$, Proposition 3.3 shows

$$
X_{\underline{w}_{m}}^{m}(1) \cong \coprod_{g \in G(F) / U_{\mathfrak{J}}} \mathrm{p}_{m}^{-1}\left(g D_{w}^{\tau}\right)=\coprod_{g \in G(F) / U_{\mathfrak{J}}} g \cdot \mathrm{p}_{m}^{-1}\left(D_{w}^{\tau}\right) .
$$

Now, Lemma 3.9 implies that $\mathrm{p}_{m}^{-1}\left(D_{w}^{\tau}\right)=X_{\underline{w}_{m}}^{m}(1) \cap C_{v}^{m}=Y_{\dot{w}}^{m}$, hence the isomorphism claimed in the theorem. As $\mathrm{p}_{m}^{-1}\left(D_{w}^{\tau}\right) \subseteq X_{\underline{w}_{m}}^{m}(1)$ is stable under the right $I_{m, \underline{w}_{m}}$ - and left $U_{\mathfrak{J}}$-actions, the above shows that $Y_{\dot{w}}^{m}$ also is. As $\tilde{I}_{m, \underline{w}_{m}}=\coprod_{i} I_{m, \underline{w}_{m}} y_{i}$, with $y_{i}:=e_{0}\left(u^{i},(-u)^{i}\right)$ the theorem now follows from Lemma 3.11 .

Lemma 3.9. Let $\dot{x} I^{m}=\psi_{\dot{v}}^{m}(a, C, D, A, B)$ be a point of $C_{v}^{m}$. Assume $m \leqslant \ell(w)=2 n-1$.

(i) Let $R:=u^{-1}(\tau(a)-a) \bmod u^{m+1}$. Then

$$
\operatorname{inv}^{m}\left(\dot{x} I^{m}, \tau\left(\dot{x} I^{m}\right)\right)=\dot{w} \Leftrightarrow\left\{\begin{array}{l}
a_{1} \neq 0 \quad \text { (i.e., } R \text { is invertible) } \\
B=u^{n} C \tau(C)^{-1} \\
D=R^{-1} \tau(C)\left(1+u^{n} C \tau(C)^{-1} A+u^{n}(-1)^{n} C^{-1} \tau(C) \tau(A)\right)
\end{array}\right.
$$

(the equations on the right hand side take place in $k[u] / u^{m+1}$ ).

(ii) Suppose, $\dot{x} I^{m}$ satisfies the equations on the right hand side of (3.13). Then:

$$
\operatorname{inv}^{m}\left(\dot{x} I^{m}, \sigma\left(\dot{x} I^{m}\right)\right)=1 \Leftrightarrow a, A, B, C, D \text { are } k \text {-rational. }
$$

Proof. Choose some lifts of $a, A, B, C, D$ to elements of $k \llbracket u \rrbracket$. We denote them by the same letters. (i): A computation shows that the $I$-double coset of $\dot{x}^{-1} \tau(\dot{x})$ is equal to the $I$-double coset of the element $e_{+}\left(u^{-n} R\right)$, and $\dot{w}$ lies in this double coset if and only if $R$ is invertible. This is clearly necessary for the left hand side of part (i) to hold. Thus we can assume in the following that $a_{1} \neq 0$, i.e., that $R$ is invertible. In $G(\breve{E})$ one easily computes (independently of the parity of $n$ ):

$$
\dot{v}^{-1} e_{-}(-a) e_{-}(\tau(a)) \tau(\dot{v})=e_{-}\left(u^{n} R^{-1}\right) e_{0}\left(R, R^{-1}\right) \dot{w} e_{-}\left((-1)^{n+1} u^{n} R^{-1}\right) .
$$

In the rest of the proof we write $x \sim y$ to express that $x, y$ lie in the same $I^{m}$-double coset. Using (3.14) we compute: 


$$
\begin{aligned}
\dot{x}^{-1} \tau(\dot{x}) & \sim e_{-}(-B) e_{+}(-A) e_{0}\left(C^{-1}, D^{-1}\right) \cdot\left[e_{-}\left(u^{n} R^{-1}\right) e_{0}\left(R, R^{-1}\right) \cdot \dot{w} \cdot e_{-}\left((-1)^{n+1} u^{n} R^{-1}\right)\right] \cdot \ldots \\
& \ldots \quad e_{0}(\tau(C), \tau(D)) e_{+}(\tau(A)) e_{-}(\tau(B)) \\
(3.15) & \sim e_{-}(-B) e_{+}(-A) e_{-}\left(u^{n} C D^{-1} R^{-1}\right) \cdot \dot{w} \cdot e_{0}\left(D^{-1} R^{-1} \tau(C), C^{-1} R \tau(D)\right) \ldots \\
\ldots & e_{-}\left((-1)^{n+1} u^{n} \tau(C) \tau(D)^{-1} R^{-1}\right) e_{+}(\tau(A)) e_{-}(\tau(B)) .
\end{aligned}
$$

Let $N:=1-u^{n} C D^{-1} R^{-1} A$. We apply formulas 3.4 to deduce:

$$
\begin{aligned}
I^{m} e_{-}(-B) e_{+}(-A) e_{-}\left(u^{n} C D^{-1} R^{-1}\right) & =I^{m} e_{-}\left(-B+u^{n} C D^{-1} R^{-1} N^{-1}\right) e_{+}(-A N) e_{0}\left(N, N^{-1}\right) \\
& =I^{m} e_{-}\left(-B+u^{n} C D^{-1} R^{-1}\right) e_{+}(-A N) e_{0}\left(N, N^{-1}\right),
\end{aligned}
$$

where the last equation is true, since $u^{n} N^{ \pm 1} \equiv u^{n} \bmod u^{m+1}$, which in turn follows from $2 n-1 \geqslant m$. Noting that the product of the last three matrices in the last expression in (3.15) is equal to $\tau$ applied to the inverse of the product of the first three (use $\tau(R)=R$ ), we deduce from 3.15 and (3.16):

$$
\begin{aligned}
\dot{x}^{-1} \tau(\dot{x}) & \sim e_{-}\left(-\left(B-u^{n} C D^{-1} R^{-1}\right)\right) e_{+}(-N A) e_{0}\left(N, N^{-1}\right) \cdot \dot{w} \cdot \ldots \\
& \ldots e_{0}\left(\tau(C) D^{-1} R^{-1}, C^{-1} \tau(D) R\right) e_{0}\left(\tau(N)^{-1}, \tau(N)\right) e_{+}(\tau(N A)) \ldots \\
& \ldots e_{-}\left(\tau\left(B-u^{n} C D^{-1} R^{-1}\right)\right) .
\end{aligned}
$$

Now we bring the term $e_{+}(-N A)$ to the right side of $\dot{w}$, without modifying the other terms and it can be canceled there, as it lands in $I^{m}$ and $I^{m}$ is normal in $I$. Here we again used $2 n-1 \geqslant m$. Analogously, we cancel the term $e_{+}(\tau(N A))$ by bringing it to the left side of $\dot{w}$. Now put the three $e_{0}$-terms together and obtain

$$
\begin{array}{lll}
\dot{x}^{-1} \tau(\dot{x}) & \sim e_{-}\left(-\left(B-u^{n} C D^{-1} R^{-1}\right)\right) \cdot \dot{w} \cdot \ldots \\
& \ldots & e_{0}\left(\tau(C) D^{-1} R^{-1} N^{-1} \tau(N)^{-1}, C^{-1} \tau(D) R N \tau(N)\right) e_{-}\left(\tau\left(B-u^{n} C D^{-1} R^{-1}\right)\right) .
\end{array}
$$

The left hand side of 3.13 is equivalent to $\dot{x}^{-1} \tau(\dot{x}) \sim \dot{w}$, which by 3.17 and Section 3.1.8 is equivalent to

$$
\begin{aligned}
& B-u^{n} C D^{-1} R^{-1} \equiv 0 \bmod u^{m+1} \\
& \tau(C) D^{-1} R^{-1} N^{-1} \tau(N)^{-1} \equiv 1 \bmod u^{m+1} \\
& C^{-1} \tau(D) R N \tau(N) \equiv 1 \bmod u^{m+1} .
\end{aligned}
$$

Using $\tau^{2}=1$ and $\tau(R)=R$, we see that the second and the third equations are equivalent. Hence the third can be ignored. Assume first $n \geqslant m+1$. Then it is trivial to see that (3.18) is equivalent to the right hand side of (3.13). Assume now $m \geqslant n$. Then, as $n \geqslant m+1-n>0$ and $N \equiv 1 \bmod u^{n}$, the second equation of (3.18) shows

$$
D \equiv \tau(C) R^{-1} \bmod u^{m+1-n} .
$$

Using this and $N=1-u^{n} C D^{-1} R^{-1} A$ it is now easy to deduce the equivalence of (3.18) and the right hand side of (3.13). 
(ii): The implication ' $\Leftarrow$ ' is immediate. We prove ' $\Rightarrow$ '. Assume $\dot{x}^{-1} \sigma(\dot{x}) \in I^{m}$. In particular, the $I$-double coset of $\dot{x}^{-1} \sigma(\dot{x})$ is $I$. This is equivalent to $a$ being $k$-rational, and we deduce $\dot{v}^{-1} e_{-}(\sigma(a)-a) \sigma(\dot{v})=1$. Setting $G:=1+A B$, we compute

$$
\begin{aligned}
& \dot{x}^{-1} \sigma(\dot{x})=e_{-}(-B) e_{+}(-A) e_{0}\left(C^{-1}, D^{-1}\right) e_{0}(\sigma(C), \sigma(D)) e_{+}(\sigma(A)) e_{-}(\sigma(B)) \\
& =\left(\begin{array}{cc}
C^{-1} \sigma(C) \sigma(G)-D^{-1} \sigma(D) \sigma(B) A & C^{-1} \sigma(C) \sigma(A)-D^{-1} \sigma(D) A \\
D^{-1} \sigma(D) G \sigma(B)-C^{-1} \sigma(C) B \sigma(G) & D^{-1} \sigma(D) G-C^{-1} \sigma(C) B \sigma(A)
\end{array}\right) .
\end{aligned}
$$

We have to show that $B, C, D$ resp. $A$ are $\sigma$-stable $\bmod u^{m+1} \operatorname{resp} . \bmod u^{m}$. If $n \geqslant m+1$, we have $B \equiv 0 \bmod u^{m+1}$ and $G \equiv 1 \bmod u^{m+1}$ by assumption and part (i), and the claimed equivalence is trivial. Assume $m \geqslant n$. By assumption and as $n \geqslant m+1-n>0$, we know that

$$
\begin{aligned}
G & =1+A B \equiv 1+u^{n} C \tau(C)^{-1} A \quad \bmod u^{m+1} \\
B & =u^{n} C \tau(C)^{-1} \equiv 0 \quad \bmod u^{n},
\end{aligned}
$$

and we deduce from 3.20

$$
\begin{aligned}
C^{-1} \sigma(C) \sigma\left(1+u^{n} C \tau(C)^{-1} A\right) & \equiv 1+D^{-1} \sigma(D) u^{n} \sigma\left(C \tau(C)^{-1}\right) A & \bmod u^{m+1} \\
C^{-1} \sigma(C) \sigma(A) & \equiv D^{-1} \sigma(D) A \quad \bmod u^{m} & \\
D^{-1} \sigma(D)\left(1+u^{n} C \tau(C)^{-1} A\right) & \equiv 1+C^{-1} \sigma(C) u^{n} C \tau(C)^{-1} \sigma(A) & \bmod u^{m+1}
\end{aligned}
$$

$$
D^{-1} \sigma(D)\left(1+u^{n} C \tau(C)^{-1} A\right) \sigma\left(u^{n} C \tau(C)^{-1}\right) \equiv \ldots
$$

$$
\cdots \equiv C^{-1} \sigma(C) u^{n} C \tau(C)^{-1}\left(1+u^{n} \sigma(C) \sigma(\tau(C))^{-1} \sigma(A)\right) \quad \bmod u^{m+1} .
$$

From (3.21), 3.23) and $m \geqslant n$, we deduce $C \equiv \sigma(C) \bmod u^{n}$ and $D \equiv \sigma(D) \bmod u^{n}$. Using this and $m \geqslant n$, we deduce from $(3.22)$ that $A \equiv \sigma(A) \bmod u^{n}$. Using these congruences and $n \geqslant m+1-n>0$, we may replace $\sigma(A), \sigma(C), \sigma(D)$ by $A, C, D$ in all terms which are $\equiv 0 \bmod u^{n}$ in equations (3.21)-(3.24). Then (3.21) simplifies to $C^{-1} \sigma(C) \equiv 1 \bmod u^{m+1}$ and (3.23 to $D^{-1} \sigma(D) \equiv 1 \bmod u^{m+1}$. Using this, we deduce $\sigma(A) \equiv A \bmod u^{m}$ from equation (3.22). The $\sigma$-stability of $B$ follows by assumption and (3.13). This finishes the proof of the lemma.

\section{Remark 3.10.}

(i) Lemma 3.9(ii) shows, that one could have started directly with $E / F$ and $\Sigma=\{\tau\}$, instead of $E / F$ and $\Sigma=\{\sigma, \tau\}$ as in the text, to obtain the same results. However, the approach in the text seems to the author to be more flexible.

(ii) The computations in the proof of Lemma 3.9 get significantly simpler under the stronger assumption $n \geqslant m+1$. However, it is the 'hardest' case $m=2 n-1$ of this theorem, which is necessary to realize the automorphic induction in a pure way, see Theorems 4.2 . 4.32 .

Lemma 3.11. $\tilde{Y}_{\dot{w}}^{m}$ is stable under the left action of $E^{\times} U_{\mathfrak{J}}$.

Proof. As $Y_{\dot{w}}^{m}$ is $U_{\mathfrak{J}}$-stable (see the proof of Theorem 3.8), $\tilde{Y}_{\dot{w}}^{m}$ also is. As $E^{\times} U_{\mathfrak{J}}$ is generated by $U_{\mathfrak{J}}$ and $\varpi(\varpi$ as in Section 3.1 .3$)$, it is enough to show $\varpi \tilde{Y}_{\dot{w}}^{m}=\tilde{Y}_{\dot{w}}^{m}$, which in turn follows from $\varpi Y_{\dot{w}}^{m}=Y_{\dot{w}}^{m} y_{1}$. Let $\mathrm{pr}_{m}: \mathscr{F}^{m} \rightarrow \mathscr{F}$ denote the natural projection. Lemma 3.12 shows $\varpi D_{w}^{\tau}=D_{w}^{\tau} y_{1}$. Using $\varpi$-(resp. $y_{1^{-}}$)equivariance of $\mathrm{pr}_{m}$ and $\varpi$-(resp. $y_{1^{-}}$)invariance of $X_{\underline{w}_{m}}^{m}(1)$, we deduce from this 


$$
\begin{aligned}
\varpi Y_{\dot{w}}^{m} & =\varpi\left(\operatorname{pr}_{m}^{-1}\left(D_{w}^{\tau}\right) \cap X_{\underline{w}_{m}}^{m}(1)\right)=\operatorname{pr}_{m}^{-1}\left(\varpi D_{w}^{\tau}\right) \cap X_{\underline{w}_{m}}^{m}(1)=\operatorname{pr}_{m}^{-1}\left(D_{w}^{\tau} y_{1}\right) \cap X_{\underline{w}_{m}}^{m}(1) \\
& =\left(\operatorname{pr}_{m}^{-1}\left(D_{w}^{\tau}\right) \cap X_{\underline{w}_{m}}^{m}(1)\right) y_{1}=Y_{\dot{w}}^{m} y_{1} .
\end{aligned}
$$

Lemma 3.12. Let $\psi_{\dot{v}}(a) \in C_{v}$ be a point. Write $a=u a^{\prime}$ and assume that $v_{u}\left(a^{\prime}\right)=0$. The point $\varpi \psi_{\dot{v}}(a) y_{1}^{-1}$ of $\mathscr{F}$ (with $y_{1}$ as in Definition 3.6) lies in $C_{v}$. Moreover,

$$
\varpi \psi_{\dot{v}}(a) y_{1}^{-1}=\psi_{\dot{v}}\left(u a^{\prime,-1}\right) .
$$

Proof. A computation shows that the $I$-cosets $\varpi e_{-}(a) \dot{v} y_{1}^{-1} I$ and $e_{-}\left(u a^{\prime,-1}\right) I$ coincide.

\section{Representation Theory}

Recall that $G=\mathrm{GL}_{2}$ and $\operatorname{char} k \neq 2$. We use the notation from Section 3 . Further, we fix a prime $\ell \neq$ char $k$. All representations considered below are smooth $\overline{\mathbb{Q}}_{\ell}$-representations.

\subsection{Some preparations.}

4.1.1. Filtrations on $U_{\mathfrak{J}}$ and $U_{E}$. Recall the $\mathcal{O}_{F}$-algebra $\mathfrak{J}$ from Section 3.1.3. Then

$$
U_{\mathfrak{J}}^{n}:=1+\varpi^{n} \mathfrak{J}=\left(\begin{array}{cc}
1+\mathfrak{p}_{F}^{\left\lfloor\frac{n+1}{2}\right\rfloor} & \mathfrak{p}_{F}^{\left\lfloor\frac{n}{2}\right\rfloor} \\
\mathfrak{p}_{F}^{\left\lfloor\frac{n}{2}\right\rfloor+1} & 1+\mathfrak{p}_{F}^{\left\lfloor\frac{n+1}{2}\right\rfloor}
\end{array}\right)
$$

for $n \geqslant 0$ form a filtration of $U_{\mathfrak{J}}^{0}:=U_{\mathfrak{J}}$ by open subgroups. Moreover, for $n \geqslant 0$, we denote by $U_{E}^{n}$ the $n$-units of $E$. Note that via $\iota$ we have $U_{\mathfrak{J}}^{n} \cap E^{\times}=U_{E}^{n}$.

4.1.2. Some notation. For a locally compact group $H$, we denote by $H^{\vee}$ the set of all smooth $\overline{\mathbb{Q}}_{\ell}^{\times}$-valued characters of $H$. For an additive character $\psi$ of $F$, we let $\psi_{E}:=\psi \circ \operatorname{tr}_{E / F}$ be the corresponding character of $E$, where $\operatorname{tr}_{E / F}$ is the trace of $E / F$. Let $\mathfrak{M}:=M_{2}\left(\mathcal{O}_{F}\right)$. We denote by $\psi_{\mathfrak{M}}:=\psi \circ \operatorname{tr}_{\mathfrak{M}}$ the corresponding character of $\mathfrak{M}$. For a character $\phi$ of $F^{\times}$we set $\phi_{E}:=\phi \circ \mathrm{N}_{E / F}$ be the corresponding character of $E^{\times}$, where $\mathrm{N}_{E / F}$ is the norm of $E / F$. For a $G(F)$-representation $\pi$ we denote by $\phi \pi$ the $G(F)$-representation $g \mapsto \phi(\operatorname{det}(g)) \pi(g)$.

4.1.3. Characters of $U_{\mathfrak{J}}$. Let $\psi$ be an additive character of $F$ of level 1 (i.e., $\psi\left(\mathfrak{p}_{F}\right)=1$, but $\psi$ non-trivial on $\left.\mathcal{O}_{F}\right)$. Let $0 \leqslant k<r \leqslant 2 k+1$ be integers. By $[\mathrm{BH} \mid 12.5$ Proposition we have isomorphisms

$$
\varpi^{-r} \mathfrak{J} / \varpi^{-k} \mathfrak{J} \stackrel{\sim}{\longrightarrow}\left(U_{\mathfrak{J}}^{k+1} / U_{\mathfrak{J}}^{r+1}\right)^{\vee}, \quad a+\left.\varpi^{-k} \mathfrak{J} \mapsto \psi_{\mathfrak{M}, a}\right|_{U_{\mathfrak{J}}^{m+1}}
$$

where $\psi_{\mathfrak{M}, a}$ denotes the function $x \mapsto \psi_{\mathfrak{M}}(a(x-1))$ and $\mathfrak{M}$ is as in Section 4.1 .2 .

4.1.4. Admissible pairs. Let $\chi$ be a character of $E^{\times}$. The level $\ell(\chi)$ of $\chi$ is the least integer $m \geqslant 0$, such that $\left.\chi\right|_{U_{E}^{m+1}}$ is trivial. The pair $(E / F, \chi)$ is said to be admissible $([\overline{\mathrm{BH}} \mid 18.2)$ if $\left.\chi\right|_{U_{E}^{1}}$ does not factor through the norm map $\mathrm{N}_{E / F}$. An admissible pair $(E / F, \chi)$ with $\chi$ of level $m$ is called minimal, if $\left.\chi\right|_{U_{E}^{m}}$ does not factor through $\mathrm{N}_{E / F}$. Note that if $(E / F, \chi)$ is minimal, then $\ell(\chi)$ is odd. Two pairs $(E / F, \chi),\left(E / F, \chi^{\prime}\right)$ are said to be $F$-isomorphic if there is some $\gamma \in \mathrm{Gal}_{E / F}$ such that $\chi^{\prime}=\chi \circ \gamma$. We denote by $\mathbb{P}_{2}^{\operatorname{tr}}(F)$ the set of isomorphism classes of all admissible pairs attached to the tamely ramified extension $E / F$. 
4.1.5. Supercuspidal representations. Denote by $\mathscr{A}_{2}^{\operatorname{tr}}(F)$ the set of all isomorphism classes of irreducible supercuspidal representations of $G(F)$, which are not unramified (i.e., are not attached to an unramified stratum. We use the definition of unramified from $[\mathrm{BH}] 20.1$, see also 20.3 Lemma). The ramified part of the tame parametrization theorem ( $[\mathrm{BH}] 20.2$ Theorem) states the existence of a certain bijection

$$
\pi: \mathbb{P}_{2}^{\operatorname{tr}}(F) \stackrel{\sim}{\rightarrow} \mathscr{A}_{2}^{\operatorname{tr}}(F) \quad(E / F, \chi) \mapsto \pi_{\chi}
$$

4.1.6. Bushnell-Henniart construction of $\pi_{\chi}$. We recall the construction of $\pi_{\chi}$ from $[\mathrm{BH}] \S 15,19$. By twisting with a character of $F^{\times}$, it is enough to construct $\pi_{\chi}$ for minimal pairs. Fix an additive character $\psi$ of $F$ of level one. Let $(E / F, \chi)$ be a minimal admissible pair with $\chi$ of odd level $m=2 n-1 \geqslant 1$. Choose an element $\beta \in \mathfrak{p}_{E}^{-m}$ such that

$$
\chi(1+x)=\psi_{E}(\beta x) \quad \text { for all } x \in \mathfrak{p}_{E}^{n} .
$$

Via $\iota$ we see $\beta$ as an element of $M_{2}(F)$. Then $(\mathfrak{J}, m, \beta)$ is a ramified simple stratum (see $[\mathrm{BH}$ 13.1). Via (4.1), $\beta$ defines a character $\psi_{\beta}$ of $U_{\mathfrak{J}}^{n}$, which is trivial on $U_{\mathfrak{J}}^{m+1}$. Let $\Lambda$ be the character of $J_{\beta}:=E^{\times} U_{\mathfrak{J}}^{n}$ defined by

$$
\left.\Lambda\right|_{U_{\mathfrak{J}}^{n}}:=\psi_{\beta},\left.\quad \Lambda\right|_{E^{\times}}:=\chi
$$

(by (4.3) this is a consistent definition, as $\left.\operatorname{tr}_{\mathfrak{M}}\right|_{\mathcal{O}_{E}}=\left.\operatorname{tr}_{E / F}\right|_{\mathcal{O}_{E}}$ and $\left.E \cap U_{\mathfrak{J}}^{n}=U_{E}^{n}\right)$. Then $\left(\mathfrak{J}, J_{\beta}, \Lambda\right)$ is a cuspidal type in $G(F)$ attached to $(E / F, \chi)$ (see $[\mathrm{BH}] 15.5$ ). The cuspidal inducing datum attached to this cuspidal type is the pair $\left(U_{\mathfrak{J}}, \Theta_{\chi}\right)$, where $\Theta_{\chi}:=\mathrm{c}-\operatorname{Ind}_{J_{\beta}}^{E^{\times} U_{\mathfrak{J}}} \Lambda$. Then $\pi_{\chi}$ is defined to be the compact induction

$$
\pi_{\chi}:=\mathrm{c}-\operatorname{Ind}_{J_{\beta}}^{G(F)} \Lambda=\mathrm{c}-\operatorname{Ind}_{E^{\times} U_{\mathfrak{J}}}^{G(F)} \Theta_{\chi} .
$$

The isomorphism class of $\pi_{\chi}$ is independent of the choices of $\iota, \psi$ and $\beta$. We work with the fixed choice of $\iota$, but $\psi$ and $\beta$ can be arbitrary.

4.1.7. Cohomology. For a scheme $X$ over $k$ we denote by $\mathrm{H}_{c}^{*}\left(X, \overline{\mathbb{Q}}_{\ell}\right)$ the $\ell$-adic cohomology of $X$ with compact support.

\subsection{Automorphic induction from the ramified torus of $\mathrm{GL}_{2}$.}

Let $m \geqslant 1$ be an odd integer. Let $\chi$ be character of $E^{\times}$of level $m$. Let $\underline{w}_{m}$ be as in Notation 3.4. By inflation via (3.11), $\chi$ determines a character of $\tilde{I}_{m, \underline{w}_{m}} / I^{m}$ and hence we can consider the $\chi$-isotypic subspace $\mathrm{H}_{c}^{*}\left(X_{\underline{w}_{m}}^{m}(1), \overline{\mathbb{Q}}\right)[\chi]$ of the cohomology of $X_{\underline{w}_{m}}^{m}(1)$. Analogously, we can consider the $\chi$-isotypic subspace in the cohomology of $\tilde{Y}_{\dot{w}}^{m}$.

Definition 4.1. Let $(E / F, \chi)$ be a minimal pair of odd level $m \geqslant 1$. Let $w, n, \underline{w}$ be as in Notation 3.1 such that $\ell(w)=2 n-1 \geqslant m$ and take $\underline{w}_{m}$ as in Notation 3.4 lying over $\underline{w}$. Define $R_{\chi, n}$ to be the $G(F)$-representation

$$
R_{\chi, n}:=\mathrm{H}_{c}^{0}\left(X_{\underline{w}_{m}}^{m}(1), \overline{\mathbb{Q}}\right)[\chi]
$$

and $\Xi_{\chi, n}$ to be the $E^{\times} U_{\mathfrak{J}}$-representation

$$
\Xi_{\chi, n}:=\mathrm{H}_{c}^{0}\left(\tilde{Y}_{\dot{w}}^{m}, \overline{\mathbb{Q}_{\ell}}\right)[\chi] .
$$

For an arbitrary admissible pair $(E / F, \chi)$ such that $\chi=\phi \chi^{\prime}$ with $\left(E / F, \chi^{\prime}\right)$ minimal we define $R_{\chi, n}:=\phi R_{\chi^{\prime}, n}, \Xi_{\chi, n}:=\phi \Xi_{\chi^{\prime}, n}$. If $m=2 n-1$, write 


$$
R_{\chi}:=R_{\chi, n} \quad \text { and } \quad \Xi_{\chi}:=\Xi_{\chi, n}
$$

We also denote by $V_{\chi}$ the space in which $\Xi_{\chi}$ acts.

As $X_{\underline{w}_{m}}^{m}(1)$ is zero-dimensional, its cohomology in all positive degrees vanishes, and Definition 4.1 is compatible with (1.1). We suppose that $R_{\chi}$ for non-minimal pairs occurs also naturally in the zeroth cohomology of $X_{\underline{w}_{m}}^{m}$ (1) with $m$ even. The following theorem is our main result.

Theorem 4.2. Let $(E / F, \chi)$ be an admissible pair. The representation $R_{\chi}$ is irreducible, cuspidal, ramified, has level $\ell(\chi)$ and central character $\left.\chi\right|_{F^{\times}}$. Moreover, $R_{\chi}$ is isomorphic to $\pi_{\chi}$, i.e., the map

$$
R: \mathbb{P}_{2}^{\operatorname{tr}}(F) \rightarrow \mathscr{A}_{2}^{\operatorname{tr}}(F) \quad(E / F, \chi) \mapsto R_{\chi}
$$

coincides with the map $\pi_{\chi}$ from (4.2) and is, in particular, a bijection.

After necessary preparations, Theorem 4.2 is shown in Sections 4.6, 4.7. We wish to point out, that the injectivity of (4.4) follows from the results of Section 4.5 and essentially does not use cuspidal types and the isomorphism $R_{\chi} \cong \pi_{\chi}$. We need them to prove surjectivity of (4.4). From Theorem 3.8 we deduce:

Lemma 4.3. Let $(E / F, \chi)$ be an admissible pair. Then

$$
R_{\chi, n}=\mathrm{c}-\operatorname{Ind}_{E^{\times} U_{\mathfrak{J}}}^{G(F)} \Xi_{\chi, n} .
$$

Proof. It follows from Theorem 3.8 and the commutativity of the left and the right group actions on $X_{\underline{w}_{m}}^{m}(1)$.

In Section 4.8 we also study the representations $R_{\chi, n}$ for $n \geqslant m+1$, where $m$ is the (odd) level of $\chi$. We determine the structure of $R_{\chi, n}$ and give a recipe how to reconstruct $\chi$ (up to $\tau$-conjugacy) from $R_{\chi, n}$.

\subsection{Unipotent traces.}

From now on and until the end of Section 4.7 we assume $2 n-1=m$.

Lemma 4.4. The central character of $R_{\chi}$ is $\left.\chi\right|_{F^{\times}}$. The subgroup $U_{\mathfrak{J}}^{m+1}$ acts trivially in $V_{\chi}$ and $V_{\chi}$ has dimension $(q-1) q^{n-1}$.

Proof. Elements of $F^{\times}$act on $X_{\underline{w}_{m}}^{m}(1)$ in the same way from the left and from the right. As $R_{\chi}$ is the $\chi$-isotypic component of $\mathrm{H}_{c}^{0}\left(X_{\underline{w}_{m}}^{m}(1), \overline{\mathbb{Q}}_{\ell}\right)$, the first statement of the lemma follows. The proof of the second statement is given in Section 5.3 .

By Lemma 4.4 we can consider $\Xi_{\chi}$ as a $E^{\times} U_{\mathfrak{J}} / U_{\mathfrak{J}}^{m+1}$-representation. Let $N_{n}$ be the finite subgroup of $E^{\times} U_{\mathfrak{J}} / U_{\mathfrak{J}}^{m+1}$, equipped with a descending filtration by subgroups $N_{n}^{i}$ for $1 \leqslant i \leqslant$ $n+1$ defined by

$$
N_{n}^{i}:=\left(\begin{array}{cc}
1 & \\
\mathfrak{p}_{F}^{i} & 1
\end{array}\right) /\left(\begin{array}{cc}
1 & \\
\mathfrak{p}_{F}^{n+1} & 1
\end{array}\right) \subseteq N_{n}:=\left(\begin{array}{cc}
1 & \\
\mathfrak{p}_{F} & 1
\end{array}\right) /\left(\begin{array}{cc}
1 & \\
\mathfrak{p}_{F}^{n+1} & 1
\end{array}\right) \subseteq U_{\mathfrak{J}} / U_{\mathfrak{J}}^{m+1}
$$

Proposition 4.5. As $N_{n}$-representations one has

$$
\Xi_{\chi} \cong \operatorname{Ind}_{1}^{N_{n}} 1-\operatorname{Ind}_{N_{n}^{n}}^{N_{n}} 1 \cong \bigoplus_{\substack{\psi \in N_{n}^{\vee} \\ \psi N_{n}^{n} \text { non-trivial }}} \psi
$$

In particular, $\Xi_{\chi}$ does not contain the trivial character on $N_{n}^{n}$. 
Proof. The proposition follows from Lemma 4.6 by comparing the traces of $N_{n}$-representations on the left and the right side.

Lemma 4.6. For $g \in N_{n}$ we have:

$$
\operatorname{tr}\left(g ; \Xi_{\chi}\right)= \begin{cases}(q-1) q^{n-1} & \text { if } g=1 \\ -q^{n-1} & \text { if } g \in N_{n}^{n} \backslash\{1\} \\ 0 & \text { if } g \in N_{n} \backslash N_{n}^{n} .\end{cases}
$$

Proof. The proof is given in Section 5.3 .

Corollary 4.7. $\Xi_{\chi}$ is irreducible as B-representation, where $B \subseteq U_{\mathfrak{J}}$ is the subgroup consisting of lower triangular matrices.

Proof. The proof is the same as the proof of [Iv2] Corollary 4.12 (using Proposition 4.5 instead of [Iv2] Proposition 4.10).

\subsection{Some character theory.}

In this section we work relative to a fixed character $\chi$ of $E^{\times}$of odd level $m \geqslant 1$. We write $\chi^{\tau}:=\chi \circ \tau$.

\subsubsection{Admissibility of $(E / F, \chi)$.}

Lemma 4.8. The following hold:

(i) $\left.\chi\right|_{U_{E}^{m}}$ does not factor through the norm $N_{E / F}$.

(ii) $\left.\chi\right|_{U_{E}^{m}} \neq\left.\chi^{\tau}\right|_{U_{E}^{m}}$.

Proof. First we show (ii): Assume $\chi\left(1+u^{m} x\right)=\chi\left(1-u^{m} x\right)$ for all $x \in k$. As $\left(1-u^{m} x\right)^{-1} \in$ $\left(1+u^{m} x\right) U_{E}^{2 m}$ and as $\chi$ has level $m \geqslant 1$, we deduce $1=\chi\left(\left(1+u^{m} x\right)^{2}\right)=\chi\left(1+u^{m} 2 x\right)$ for all $x \in k$. As char $E \neq 2$, we obtain a conradiction to our assumption $\ell(\chi)=m$. Now we deduce (i) from (ii): assume that $\left.\chi\right|_{U_{E}^{m}}$ factors through the norm, i.e., $\chi=\chi^{\prime} \circ N_{E / F}$ on $U_{E}^{m}$. Then $\chi^{\tau}(x)=\chi^{\prime}\left(\mathrm{N}_{E / F}((\tau(x)))=\chi^{\prime}\left(N_{E / F}(x)\right)=\chi(x)\right.$, which contradicts (ii).

4.4.2. Filtration on $U_{E}$. We have the disjoint decomposition

$$
U_{E}=U_{F} U_{E}^{m+1} \cup \bigcup_{\alpha=0}^{n-1}\left(U_{F} U_{E}^{2 \alpha+1} \backslash U_{F} U_{E}^{2 \alpha+3}\right) .
$$

Note that $U_{F} U_{E}^{2 \alpha+1}=U_{F} U_{E}^{2 \alpha}$.

4.4.3. Index of coincidence for characters.

Definition 4.9. For a character $\theta$ of $E^{\times}$, which coincides with $\chi$ on $F^{\times} U_{E}^{m+1}$, we define the integer $i(\theta)=i_{\chi}(\theta)$ to be the smallest integer $i \geqslant 0$, such that $\left.\theta\right|_{F^{\times} U_{E}^{i}}=\left.\chi\right|_{F^{\times} U_{E}^{i}}$ or $\left.\theta\right|_{F^{\times} U_{E}^{i}}=$ $\left.\chi^{\tau}\right|_{F^{\times} U_{E}^{i}}$

Observe that $0 \leqslant i(\theta) \leqslant m+1$ and $i(\theta)$ is always even.

4.4.4. Modifications of characters. Fix some integer $0 \leqslant \alpha<n$. Consider the $k$-algebra

$$
R_{\alpha}:=\mathcal{O}_{E} / \mathfrak{p}_{E}^{m-2 \alpha}=k[u] / u^{m-2 \alpha} .
$$

The $\tau$-invariants of it are $R_{\alpha}^{\langle\tau\rangle}=k[t] / t^{n-\alpha}$. Consider the subset

$$
R_{\alpha}^{\langle\tau\rangle, \prime}:=\left\{s \in R_{\alpha}^{\langle\tau\rangle}: s \equiv \pm 1 \quad \bmod u^{m+1-2(2 \alpha+1)}\right\}
$$

of $R_{\alpha}^{\langle\tau\rangle}$ (note that $R_{\alpha}^{\langle\tau\rangle, \prime}=R_{\alpha}^{\langle\tau\rangle}$ if $2 \alpha+1 \geqslant n$, or equivalently, $\alpha \geqslant\left\lfloor\frac{n}{2}\right\rfloor$ ). 
Proposition 4.10. Let $0 \leqslant \alpha<n$. Let $s \in R_{\alpha}^{\langle\tau\rangle, I}$. There is a unique character $\chi_{s}$ of $F^{\times} U_{E}^{2 \alpha+1}$, such that the following hold:

(i) $\chi_{s}$ coincides with $\chi$ on $F^{\times} U_{E}^{m+1}$.

(ii) if $\alpha<\left\lfloor\frac{n}{2}\right\rfloor$, then $\chi_{s}$ coincides on $F^{\times} U_{E}^{2(2 \alpha+1)}$ with $\chi \circ \tau^{i}$, where $s \equiv(-1)^{i} \bmod u$.

(iii) $\chi_{s}\left(1+u^{2 \alpha+1} h\right)=\chi\left(1+u^{2 \alpha+1} h s\right)$ for all $h \in \mathcal{O}_{F}$.

Conversely, let $\theta$ be a character of $F^{\times} U_{E}^{2 \alpha+1}$, which coincides with $\chi$ or $\chi^{\tau}$ on $F^{\times} U_{E}^{\min \{m+1,2(2 \alpha+1)\}}$. Then there is a unique $s \in R_{\alpha}^{\langle\tau\rangle, I}$ such that $\theta=\chi_{s}$.

Note that the expression $\chi\left(1+u^{2 \alpha+1} h s\right)$ in (iii) is well-defined: Indeed, $\chi$ is trivial on $U_{E}^{m+1}$, and on the other hand if $\tilde{s}_{1}, \tilde{s}_{2} \in \mathcal{O}_{F}=k \llbracket t \rrbracket \subseteq k \llbracket u \rrbracket$ represent the same element $s$ in $R_{\alpha}^{\langle\tau\rangle,}$, then $\tilde{s}_{1} \equiv \tilde{s}_{2} \bmod u^{m-2 \alpha}$, hence $1+u^{2 \alpha+1} h \tilde{s}_{1} \equiv 1+u^{2 \alpha+1} h \tilde{s}_{2} \bmod u^{m+1}$.

Proof. Consider the subset

$$
U_{E}^{2 \alpha+1, \prime}:=\left\{x \in U_{E}^{2 \alpha+1}: \exists h \in \mathcal{O}_{F} \text { with } x \equiv 1+u^{2 \alpha+1} h \quad \bmod U_{E}^{m+1}\right\} \subseteq U_{E}^{2 \alpha+1} .
$$

Lemma 4.11. Any element $x \in F^{\times} U_{E}^{2 \alpha+1}$ can be written as $x=z_{x} x^{\prime}$ with $z_{x} \in U_{F}, x^{\prime} \in U_{E}^{2 \alpha+1, \prime}$. Moreover, modulo $U_{E}^{m+1}, z_{x}, x^{\prime}$ are uniquely determined by $x$ and if $x=\sum_{i \geqslant 0} x_{i} u^{i} \in U_{E}^{2 \alpha+1}$, then $z_{x} \equiv \sum_{i \geqslant 0} x_{2 i} u^{2 i} \bmod u^{m+1}$.

Proof. Multiplying by an element in $F^{\times}$, we can assume $x \in U_{E}^{2 \alpha+1}$. Write $x=1+\sum_{i=2 \alpha+1}^{m} x_{i} u^{i}+$ $\mathscr{O}\left(u^{m+1}\right)$. As $x^{\prime}$ has to lie in $U_{E}^{2 \alpha+1, \prime} \subseteq U_{E}^{2 \alpha+1}$, also $z_{x}$ must lie in $U_{E}^{2 \alpha+1}$. Thus we seek for two elements $z_{x}:=1+\sum_{i=\alpha+1}^{n-1} z_{2 i} u^{2 i}+\mathscr{O}\left(u^{m+1}\right)$ and $x^{\prime}=1+\sum_{i=\alpha}^{n-1} y_{2 i+1} u^{2 i+1}+\mathscr{O}\left(u^{m+1}\right)$ which have to satisfy

$$
\left(1+\sum_{i=\alpha+1}^{n-1} z_{2 i} u^{2 i}+\mathscr{O}\left(u^{m+1}\right)\right)\left(1+\sum_{i=\alpha}^{n-1} y_{2 i+1} u^{2 i+1}+\mathscr{O}\left(u^{m+1}\right)\right)=1+\sum_{i=2 \alpha+1}^{m} x_{i} u^{i}+\mathscr{O}\left(u^{m+1}\right) .
$$

Comparing the parity of the degrees we see that $z_{2 i}=x_{2 i}$. Further, a computation shows that $y_{i}$ 's satisfying this equation exist and are uniquely determined by the $x_{i}$ 's.

Let now $s \in R_{\alpha}^{\langle\tau\rangle, \prime}$. For $x \in F^{\times} U_{E}^{2 \alpha+1}$ with decomposition $x=z_{x} x^{\prime}$ according to Lemma 4.11 set

$$
\chi_{s}(x):=\chi\left(z_{x}\right) \chi\left(1+u^{2 \alpha+1} h\right) \quad \text { where } x^{\prime}=1+u^{2 \alpha+1} h \text { with } h \in \mathcal{O}_{F} .
$$

We show that $\chi_{s}$ is a character of $F^{\times} U_{E}^{2 \alpha+1}$. Let $x, y \in F^{\times} U_{E}^{2 \alpha+1}$ with decompositions $x=z_{x} x^{\prime}$, $y=z_{y} y^{\prime}$ as in Lemma 4.11 and let $x^{\prime}=1+u^{2 \alpha+1} h_{x}, y^{\prime}=1+u^{2 \alpha+1} h_{y}$ (up to some elements in $\left.U_{E}^{m+1}\right)$. Write $A:=u^{2 \alpha+1}\left(h_{x}+h_{y}\right), B:=u^{2(2 \alpha+1)} h_{x} h_{y}$. We compute

$$
\begin{aligned}
\chi_{s}(x) \chi_{s}(y) & =\chi\left(z_{x} z_{y}\left(1+u^{2 \alpha+1} s h_{x}\right)\left(1+u^{2 \alpha+1} s h_{y}\right)\right) \\
& =\chi\left(z_{x} z_{y}\left(1+s A+s^{2} B\right)\right) \\
& =\chi\left(z_{x} z_{y}(1+s A+B)\right)
\end{aligned}
$$

the last equation being true, as $s^{2} \equiv 1 \bmod u^{m+1-2(2 \alpha+1)}$. We have

$$
x^{\prime} y^{\prime}=1+A+B .
$$

As $h_{x}, h_{y} \in \mathcal{O}_{F}$, Lemma 4.11 implies $z_{x^{\prime} y^{\prime}}=1+B$ (up to elements in $U_{E}^{m+1}$ ). We deduce

$$
\left(x^{\prime} y^{\prime}\right)^{\prime}=x^{\prime} y^{\prime} z_{x^{\prime} y^{\prime}}^{-1}=1+A-A B(1+B)^{-1} .
$$

Now, $x y=z_{x} z_{y} z_{x^{\prime} y^{\prime}}\left(x^{\prime} y^{\prime}\right)^{\prime}$ is the decomposition of $x y$ according to Lemma 4.11 and we compute 


$$
\chi_{s}(x y)=\chi\left(z_{x} z_{y} z_{x^{\prime} y^{\prime}}\right) \chi\left(1+s\left(A-A B(1+B)^{-1}\right)\right) .
$$

If $2 \alpha+1 \geqslant n$, we have $B \in \mathscr{O}\left(u^{m+1}\right)$, hence all terms containing $B$ can be ignored and we deduce $\chi_{s}(x) \chi_{s}(y)=\chi_{s}(x y)$. Assume $2 \alpha+1<n$. Let $\operatorname{sgn}(s):= \pm 1$, if $s \equiv \pm 1 \bmod u$. From the above, $s \equiv(-1)^{\operatorname{sgn}(s)} \bmod u^{m+1-2(2 \alpha+1)}$ and $B \equiv 0 \bmod u^{2(2 \alpha+1)}$ we deduce

$$
\begin{aligned}
\chi_{s}(x y) & =\chi\left(z_{x} z_{y}(1+B)\left(1+s A-(-1)^{\operatorname{sgn}(s)} A B(1+B)^{-1}\right)\right) \\
& =\chi\left(z_{x} z_{y}\left(1+s A+B+s A B-(-1)^{\operatorname{sgn}(s)} A B\right)\right) \\
& =\chi\left(z_{x} z_{y}(1+s A+B)\right)
\end{aligned}
$$

This shows that $\chi_{s}$ is a character. Now, $\chi_{s}$ satisfies (i) and (iii) by definition and (ii) is immediate. This finishes the proof of the first part of the proposition. For the converse statement, one shows by a simple computation that the map $s \mapsto \chi_{s}$ from $R_{\alpha}^{\langle\tau\rangle, I}$ to characters of $F^{\times} U_{E}^{2 \alpha+1}$ is injective. This completes the proof, as the number of elements in $R_{\alpha}^{\langle\tau\rangle, \prime}$ coincides with the number of characters $\theta$ of $F^{\times} U_{E}^{2 \alpha+1}$, which are equal to $\chi$ or $\chi^{\tau}$ on $F^{\times} U_{E}^{\min \{m+1,2(2 \alpha+1)\}}$ (if $2 \alpha+1 \geqslant n$, then there are $q^{n-\alpha}$ those, otherwise there are $2 q^{\alpha+1}$ ).

4.4.5. Compatibility with changing $\alpha$. Let $0 \leqslant \alpha<n$. Let $\theta$ be a character of $E^{\times}$, coinciding on $F^{\times} U_{E}^{\min \{m+1,2(2 \alpha+1)\}}$ with $\chi$ or $\chi^{\tau}$. By Proposition 4.10 , there is some $s(\theta, \alpha) \in R_{\alpha}^{\langle\tau\rangle, I}$ such that $\left.\theta\right|_{F^{\times} U_{E}^{2 \alpha+1}}=\chi_{s(\theta, \alpha)}$. This construction is compatible with changing the level $\alpha$.

Lemma 4.12. Let $0 \leqslant \alpha_{1} \leqslant \alpha_{2}<n$. Let $\theta$ be a character of $E^{\times}$coinciding on $F^{\times} U_{E}^{\min \left\{m+1,2\left(2 \alpha_{1}+1\right)\right\}}$ with $\chi$ or $\chi^{\tau}$. Under the natural projection $R_{\alpha_{1}}^{\langle\tau\rangle} \rightarrow R_{\alpha_{2}}^{\langle\tau\rangle}, s\left(\theta, \alpha_{1}\right)$ maps to $s\left(\theta, \alpha_{2}\right)$.

Proof. Let $\bar{s}_{1}$ denote the image of $s_{1}$ in $R_{\alpha_{2}}^{\langle\tau\rangle, \prime}$. On $F^{\times} U_{E}^{2 \alpha_{2}+1}$ we have

$$
\theta\left(1+u^{2 \alpha_{2}+1} h\right)=\chi_{s_{1}}\left(1+u^{2 \alpha_{2}+1} h\right)=\chi\left(1+u^{2 \alpha_{2}+1} \bar{s}_{1} h\right) .
$$

Thus on $F^{\times} U_{E}^{2 \alpha_{2}+1}$ we have $\chi_{s_{2}}=\theta=\chi_{\bar{s}_{1}}$. By the uniqueness statement in Proposition 4.10 we have $\bar{s}_{1}=s_{2}$.

\subsubsection{Elementary modifications and distances.}

Definition 4.13. For $s \in R_{\alpha}^{\langle\tau\rangle, \prime}$, we call the character $\chi_{s}$ of $F^{\times} U_{E}^{2 \alpha+1}$ constructed in Proposition 4.10 an elementary modification of $\chi$. Let $\theta$ be character of $E^{\times}$coinciding with $\chi$ on $F^{\times} U_{E}^{m+1}$. Set

$$
\alpha_{\theta}:=\min \{\alpha: 0 \leqslant \alpha<n, 2(2 \alpha+1) \geqslant i(\theta)\},
$$

i.e., $\alpha_{\theta}$ is the smallest integer such that $\theta$ restricted to $F^{\times} U_{E}^{2 \alpha_{\theta}+1}$ is an elementary modification of $\chi$. We define the distance from $\chi$ to $\theta$ to be the (uniquely determined by Proposition 4.10)

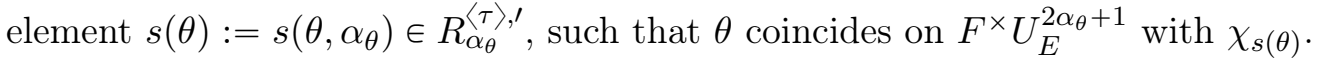

As $i(\theta)$ is an even integer $\leqslant m+1=2 n$, it follows easily that in any case $\alpha_{\theta} \leqslant\left\lfloor\frac{n}{2}\right\rfloor$. (Moreover, one has $\alpha_{\theta}=\left\lfloor\frac{i(\theta)}{4}\right\rfloor$, but we will not use this). Further, $\alpha_{\chi}=\alpha_{\chi^{\tau}}=0$ and $s(\chi)=1$ and $s\left(\chi^{\tau}\right)=-1$.

4.4.7. Quadratic distance. Let $0 \leqslant \alpha<n$. There is the norm map

$$
\mathrm{N}_{\tau, \alpha}: R_{\alpha} \rightarrow R_{\alpha}^{\langle\tau\rangle} \quad s \mapsto s \tau(s) .
$$

Lemma 4.14. The image $\operatorname{im}\left(N_{\tau, \alpha}\right)$ of $\mathrm{N}_{\tau, \alpha}$ consists of precisely such elements $s \in R_{\alpha}^{\langle\tau\rangle}$ for which $(-1)^{v_{t}(s)} \cdot s_{v_{t}(s)}$ is a square in $k^{\times}$, where $s_{v_{t}(s)}$ denotes the leading coefficient of $s$. 
Proof. This follows immediately from Lemma 4.15 .

Lemma 4.15. Let $x \in k \llbracket t \rrbracket \backslash\{0\}$ with leading coefficient $x_{v_{t}(x)} \in k^{\times}$. Then $x$ is

- a square of an element of $k \llbracket t \rrbracket$ if and only if $v_{t}(x)$ is even and $x_{v_{t}(x)}$ is a square in $k^{\times}$,

- a square of an element of $k \llbracket u \rrbracket$ if and only if $x_{v_{t}(x)}$ is a square in $k^{\times}$,

- in the image of the norm map $\mathrm{N}_{E / F}$ if and only if $(-1)^{v_{t}(x)} x_{v_{t}(x)}$ is a square in $k^{\times}$.

Proof. This is well-known.

Consider the following subset of $R_{\alpha}^{\langle\tau\rangle,}$ :

$$
Q_{\alpha}:=\left\{s \in R_{\alpha}^{\langle\tau\rangle, \prime}: s \tau(s)-1 \in \mathrm{N}_{\tau, \alpha} R_{\alpha}\right\} \backslash\{ \pm 1\} .
$$

Definition 4.16. Let $\theta$ be a character of $E^{\times}$coinciding with $\chi$ on $F^{\times} U_{E}^{m+1}$. We say that the distance from $\chi$ to $\theta$ is properly quadratic if $s(\theta) \in Q_{\alpha_{\theta}}$, with $s(\theta)$ as in Definition 4.13 .

4.4.8. Structure of $Q_{\alpha}$. Set $R_{n}^{\langle\tau\rangle}:=\{1\}$. Let $\operatorname{pr}_{\alpha}$ be the natural projection

$$
\operatorname{pr}_{\alpha}: R_{\alpha}^{\langle\tau\rangle} \cong k[t] / t^{n-\alpha} \rightarrow k[t] / t^{n-\alpha-1} \cong R_{\alpha+1}^{\langle\tau\rangle} .
$$

Lemma 4.17. Let $0 \leqslant \alpha \leqslant n-1$. An element $s \in R_{\alpha}^{\langle\tau\rangle} \backslash\{ \pm 1\}$ lies in $Q_{\alpha}$ if and only if either $-\alpha \geqslant\left\lfloor\frac{n}{2}\right\rfloor, s \not \equiv \pm 1 \bmod u$ and $s^{2}-1 \bmod u$ is a square in $k^{\times}$, or

- $s \equiv \pm 1 \bmod u$, i.e., $s= \pm 1+t^{j} s_{0}+\mathscr{O}\left(t^{j+1}\right)$ for some $s_{0} \in k^{\times}, \max \{1, n-(2 \alpha+1)\} \leqslant$ $j \leqslant n-\alpha-1$ and $\pm(-1)^{j} 2 s_{0}$ is a square in $k^{\times}$.

Moreover, the following hold:

(i) Let $0 \leqslant \alpha \leqslant n-2$. The preimage of 1 (resp. -1) under the composed map $Q_{\alpha} \hookrightarrow$ $R_{\alpha}^{\langle\tau\rangle} \rightarrow R_{\alpha+1}^{\langle\tau\rangle}$ contains precisely $\frac{q-1}{2}$ elements.

(ii) Assume $0 \leqslant \alpha \leqslant n-2$. Let $s_{0} \in R_{\alpha}^{\langle\tau\rangle, I}$ with $\operatorname{pr}_{\alpha}\left(s_{0}\right) \neq \pm 1$. Then $\sharp \operatorname{pr}_{\alpha}^{-1}\left(\operatorname{pr}_{\alpha}\left(s_{0}\right)\right)=q$ and we have the equivalence $s_{0} \in Q_{\alpha} \Leftrightarrow \operatorname{pr}_{\alpha}^{-1}\left(\operatorname{pr}_{\alpha}\left(s_{0}\right)\right) \subseteq Q_{\alpha}$.

(iii) We have $\sharp Q_{n-1}=\frac{q-3}{2}$.

Proof. The description of $Q_{\alpha}$ follows by an easy computation from Lemma 4.15. (i),(ii) follow from this description (along with $\sharp k^{\times, 2}=\frac{q-1}{2}$ ).

(iii): Note that $Q_{n-1}=\left\{s \in k: s^{2}-1\right.$ is a square in $\left.k\right\} \backslash\{ \pm 1\}$. Consider the affine curve $C: s^{2}-1=y^{2}$ over $k$ and let $\bar{C}$ be the unique smooth projective curve over $k$ containing $C$ as an open subset. We have $\sharp(\bar{C}(k) \backslash C(k))=2$. Further, $\bar{C}$ is a smooth quadric in $\mathbb{P}^{2}$ over a finite field, hence isomorphic to $\mathbb{P}^{1}$, i.e., $\sharp \bar{C}(k)=q+1$. We deduce $\sharp C(k)=q-1$. Now (iii) follows from the fact that the map $C(k) \backslash\{( \pm 1,0)\} \rightarrow Q_{n-1}$ given by $(s, y) \mapsto s$ is surjective and two-to-one.

4.5. Restriction to the ramified torus $E^{\times} \subseteq G(F)$.

For a finite finite group $H$, let $\langle,\rangle_{H}$ denote the inner product on the set of class functions of $H$. For a character $\theta$ let $\left\langle\theta, \Xi_{\chi}\right\rangle_{E^{\times}}$denote the multiplicity of $\theta$ in $\Xi_{\chi}$.

Theorem 4.18. Let $(E / F, \chi)$ be a minimal pair of odd level $m \geqslant 1$. A character $\theta$ of $E^{\times}$can only occur in $\Xi_{\chi}$, if $\theta$ coincides with $\chi$ on $F^{\times} U_{E}^{m+1}$. In this case we have

$$
\left\langle\theta, \Xi_{\chi}\right\rangle_{E^{\times}}= \begin{cases}1 & \text { if } \theta=\chi \text { or } \theta=\chi^{\tau} \text { or the distance from } \chi \text { to } \theta \text { is properly quadratic } \\ 0 & \text { otherwise. }\end{cases}
$$

We prove this theorem below. First we investigate the restriction of $\Xi_{\chi}$ to $U_{E}$. Note that $s(\theta)$ from Definition 4.13 is in exactly the same way also defined for characters $\theta$ of $U_{E}$, which coincide with $\chi$ on $U_{F} U_{E}^{m+1}$. 
Proposition 4.19. Let $(E / F, \chi)$ be a minimal pair of odd level $m \geqslant 1$. A character $\theta$ of $U_{E}$ can only occur in $\left.\Xi_{\chi}\right|_{U_{E}}$, if $\theta$ coincides with $\chi$ on $U_{F} U_{E}^{m+1}$. In this case we have

$$
\left\langle\theta, \Xi_{\chi}\right\rangle_{U_{E}}= \begin{cases}1 & \text { if } \theta=\chi \text { or } \chi^{\tau} \\ 2 & \text { if } \theta \neq \chi, \chi^{\tau} \text { and } s(\theta) \in Q_{\alpha_{\theta}} \\ 0 & \text { otherwise. }\end{cases}
$$

The main ingredient in the proof of Proposition 4.19 is the following trace computation.

Proposition 4.20. Let $0 \leqslant \alpha<n$. Let $g \in U_{F} U_{E}^{2 \alpha+1} \backslash U_{F} U_{E}^{2 \alpha+3}$. Then

$$
\operatorname{tr}\left(g ; \Xi_{\chi}\right)=2 q^{\alpha} \sum_{s \in Q_{\alpha}} \chi_{s}(g)+q^{\alpha}\left(\chi(g)+\chi^{\tau}(g)\right) .
$$

Proof. We can write $g=z g^{\prime}$ for $z \in U_{F} U_{E}^{m+1}$ and $g^{\prime}=\iota\left(1+u^{2 \alpha+1} y\right)$ with $y \in U_{F}$. Then the result follows from Proposition 5.12 applied to $g^{\prime}$ and the fact that $z$ acts in $V_{\chi}$ as the scalar $\chi(z)$ by Lemma 4.4 .

Proof of Proposition 4.19. As $U_{F} U_{E}^{m+1}$ acts in $V_{\chi}$ by $\left.\chi\right|_{U_{F} U_{E}^{m+1}}$, the first statement is clear. Assume $\left.\theta\right|_{U_{F} U_{E}^{m+1}}=\left.\chi\right|_{U_{F} U_{E}^{m+1}}$. Now, $U_{E}^{m+1}$ acts trivial in $V_{\chi}$, thus we can equivalently consider $V_{\chi}$ as a $U_{E} / U_{E}^{m+1}$-representation. The filtration from Section 4.4 .2 induces a disjoint decomposition

$$
U_{E} / U_{E}^{m+1}=Z \cup \bigcup_{\alpha=0}^{n-1}\left(H^{\alpha} \backslash H^{\alpha+1}\right),
$$

where $H^{\alpha}:=U_{F} U_{E}^{2 \alpha+1} / U_{E}^{m+1}$ and $Z:=H^{n}=U_{F} U_{E}^{m+1} / U_{E}^{m+1}$. We have $\sharp H^{\alpha}=(q-1) q^{m-\alpha}$ for $0 \leqslant \alpha \leqslant n$. For $0 \leqslant \alpha<n$ set

$$
S_{\alpha}:=\sum_{g \in H^{\alpha} \backslash H^{\alpha+1}} \theta\left(g^{-1}\right) \operatorname{tr}\left(g ; \Xi_{\chi}\right) .
$$

Then the trace computation Proposition 4.20 shows that $S_{\alpha}=(q-1) q^{m-1} S_{\alpha}^{\prime}$ with

$$
S_{\alpha}^{\prime}:=2 \sum_{s \in Q_{\alpha}}\left(q\left\langle\theta, \chi_{s}\right\rangle_{H^{\alpha}}-\left\langle\theta, \chi_{s}\right\rangle_{H^{\alpha+1}}\right)+q\left\langle\theta, \chi+\chi^{\tau}\right\rangle_{H^{\alpha}}-\left\langle\theta, \chi+\chi^{\tau}\right\rangle_{H^{\alpha+1}},
$$

and using Lemma 4.4 we deduce $\left(H^{0}=U_{E} / U_{E}^{m+1}\right)$

$$
\begin{aligned}
\left\langle\theta, \Xi_{\chi}\right\rangle_{H^{0}} & =\frac{1}{\sharp H^{0}}\left(\sum_{g \in Z} \theta\left(g^{-1}\right) \operatorname{tr}\left(g ; \Xi_{\chi}\right)+\sum_{\alpha=0}^{n-1} S_{\alpha}\right)=\frac{1}{\sharp H^{0}}\left(\sharp Z \cdot \operatorname{dim} V_{\chi}+\sum_{\alpha=0}^{n-1} S_{\alpha}\right) \\
& =\frac{1}{q}\left(q-1+\sum_{\alpha=0}^{n-1} S_{\alpha}^{\prime}\right) .
\end{aligned}
$$

Now the proposition follows from Lemma 4.21 , by considering the five cases $i(\theta)=0,0<i(\theta)<$ $m+1$ and $s(\theta) \in Q_{\alpha_{\theta}}, 0<i(\theta)<m+1$ and $s(\theta) \notin Q_{\alpha_{\theta}}, i(\theta)=m+1$ and $s(\theta) \in Q_{\alpha_{\theta}}$, $i(\theta)=m+1$ and $s(\theta) \notin Q_{\alpha_{\theta}}$.

Lemma 4.21. (i) Assume $0 \leqslant \alpha \leqslant n-2$. Then

$$
S_{\alpha}^{\prime}= \begin{cases}0 & \text { if } i(\theta) \leqslant 2 \alpha+1 \\ q & \text { if } i(\theta)=2 \alpha+2 \text { and } s(\theta, \alpha) \in Q_{\alpha} \\ -q & \text { if } i(\theta)=2 \alpha+2 \text { and } s(\theta, \alpha) \notin Q_{\alpha} \\ 0 & \text { if } i(\theta) \geqslant 2 \alpha+3 .\end{cases}
$$


(ii) For $\alpha=n-1$ we have

$$
S_{n-1}^{\prime}= \begin{cases}1 & \text { if } i(\theta) \leqslant m=2 n-1 \\ q+1 & \text { if } i(\theta)=m+1=2 n \text { and } s(\theta, n-1) \in Q_{n-1} \\ -q+1 & \text { if } i(\theta)=m+1=2 n \text { and } s(\theta, n-1) \notin Q_{n-1} .\end{cases}
$$

Proof. Let $\operatorname{pr}_{\alpha}$ be as in Section 4.4.8. (i): Assume $0 \leqslant \alpha \leqslant n-2$.

Case $i(\theta) \leqslant 2 \alpha+1$. Then on $H^{\alpha}$ resp. on $H^{\alpha+1}$ the character $\theta$ is equal to exactly one of the characters $\chi$ or $\chi^{\tau}$ (as $\alpha \leqslant n-2$ and $\chi \neq \chi^{\tau}$ on $H^{n-1}$ by Lemma 4.8). Assume that this character is $\chi$ (the other case is similar). Thus $\left\langle\theta, \chi_{s}\right\rangle_{H^{\alpha}}=0$ for all $s \in Q_{\alpha},\left\langle\theta, \chi+\chi^{\tau}\right\rangle_{H^{\alpha}}=$ $\left\langle\theta, \chi+\chi^{\tau}\right\rangle_{H^{\alpha+1}}=1$ and by Lemma 4.12 we have

$$
\left\langle\theta, \chi_{s}\right\rangle_{H^{\alpha+1}}= \begin{cases}1 & \text { if } \operatorname{pr}_{\alpha}(s)=1 \\ 0 & \text { otherwise. }\end{cases}
$$

By Lemma 4.17(i), the first case happens for exactly $(q-1) / 2$ elements in $s \in Q_{\alpha}$. Altogether we obtain $S_{\alpha}^{\prime}=2\left(0-\frac{q-1}{2}\right)+q-1=0$.

Case $i(\theta)=2 \alpha+2$. The character $\theta$ coincides on $H^{\alpha}$ neither with $\chi$ nor with $\chi^{\tau}$, hence $\left\langle\theta, \chi+\chi^{\tau}\right\rangle_{H^{\alpha}}=0$. As $2 \alpha+3 \leqslant 2(n-2)+3=m$ by assumption, $\theta$ coincides on $H^{\alpha+1}$ with precisely one of the characters $\chi$ or $\chi^{\tau}$ and hence $\left\langle\theta, \chi+\chi^{\tau}\right\rangle_{H^{\alpha+1}}=1$. As $2(2 \alpha+1) \geqslant 2 \alpha+2=i(\theta)$, the quantity $s(\theta, \alpha) \in R_{\alpha}^{\langle\tau\rangle, \prime}$ is well-defined. Thus

$$
\sum_{s \in Q_{\alpha}} q\left\langle\theta, \chi_{s}\right\rangle_{H^{\alpha}}= \begin{cases}q & \text { if } s(\theta, \alpha) \in Q_{\alpha} \\ 0 & \text { otherwise. }\end{cases}
$$

Moreover, 4.6 holds also in this case, and again there are precisely $(q-1) / 2$ elements of $Q_{\alpha}$ with image 1 in $R_{\alpha+1}^{\langle\tau\rangle}$. From this we deduce the result.

Case $i(\theta) \geqslant 2 \alpha+3$. Then $\left\langle\theta, \chi+\chi^{\tau}\right\rangle_{H^{\alpha}}=\left\langle\theta, \chi+\chi^{\tau}\right\rangle_{H^{\alpha+1}}=0$. Assume first $2(2 \alpha+1) \geqslant i(\theta)$ (in particular, $\alpha>0$ ). By Proposition 4.10 there is a unique $s(\theta, \alpha) \in R_{\alpha}^{\langle\tau\rangle, \prime}$ such that $\theta$ coincides with $\chi_{s(\theta, \alpha)}$ on $H^{\alpha}$. Hence

$$
\sum_{s \in Q_{\alpha}} q\left\langle\theta, \chi_{s}\right\rangle_{H^{\alpha}}= \begin{cases}q & \text { if } s(\theta, \alpha) \in Q_{\alpha} \\ 0 & \text { otherwise. }\end{cases}
$$

On the other hand, note that $\theta$ coincides with $\chi_{s}$ on $H^{\alpha+1}$ if and only if $s \in \operatorname{pr}_{\alpha}^{-1}\left(\operatorname{pr}_{\alpha}(s(\theta, \alpha))\right)$. Thus using Lemma 4.17(ii) we deduce

$$
\sum_{s \in Q_{\alpha}}\left\langle\theta, \chi_{s}\right\rangle_{H^{\alpha+1}}= \begin{cases}q & \text { if } s(\theta, \alpha) \in Q_{\alpha} \\ 0 & \text { otherwise. }\end{cases}
$$

In any case we compute $S_{\alpha}^{\prime}=0$. Finally, assume that $i(\theta)>2(2 \alpha+1)$, i.e., $i(\theta) \geqslant 2(2 \alpha+2)$ as $i(\theta)$ is even. Thus $\theta$ does not coincide with $\chi$ or $\chi^{\tau}$ on $U_{F} U_{E}^{\min \{m+1,2(2 \alpha+1)\}} / U_{E}^{m+1}=H^{2 \alpha+1}$. On the other hand, for $s \in R_{\alpha}^{\langle\tau\rangle, \prime}$, the character $\chi_{s}$ coincides by definition with $\chi$ or $\chi^{\tau}$ on $H^{2 \alpha+1}$. Thus $\theta$ does not coincide with any of the characters $\chi_{s}$ on $H^{2 \alpha+1}$ and from $2 \alpha+1 \leqslant 2 \alpha+3 \leqslant 4 \alpha+3$ we deduce

and hence also $S_{\alpha}^{\prime}=0$.

$$
\left\langle\theta, \chi_{s}\right\rangle_{H^{\alpha}}=\left\langle\theta, \chi_{s}\right\rangle_{H^{\alpha+1}}=0
$$


(ii): Case $i(\theta) \leqslant m$. Then $\theta$ coincides with exactly one of the characters $\chi, \chi^{\tau}$ on $H^{n-1}$. Thus $\left\langle\theta, \chi+\chi^{\tau}\right\rangle_{H^{n-1}}=1,\left\langle\theta, \chi+\chi^{\tau}\right\rangle_{Z}=2$, and $\left\langle\theta, \chi_{s}\right\rangle_{H^{n-1}}=0,\left\langle\theta, \chi_{s}\right\rangle_{Z}=1$ for all $s \in Q_{\alpha}$. Using Lemma 4.17(iii) we compute

$$
S_{n-1}^{\prime}=2\left(q \cdot 0-\frac{q-3}{2}\right)+(q \cdot 1-2)=1 .
$$

Case $i(\theta)=m+1$. Then $\left\langle\theta, \chi+\chi^{\tau}\right\rangle_{H^{n-1}}=0,\left\langle\theta, \chi+\chi^{\tau}\right\rangle_{Z}=2,\left\langle\theta, \chi_{s}\right\rangle_{Z}=1$ for all $s \in Q_{n-1}$. Moreover, $s(\theta, n-1)$ is well-defined and

$$
\sum_{s \in Q_{n-1}} q\left\langle\theta, \chi_{s}\right\rangle_{H^{n-1}}= \begin{cases}q & \text { if } s(\theta, n-1) \in Q_{n-1} \\ 0 & \text { otherwise. }\end{cases}
$$

Again we conclude by Lemma 4.17 (iii).

Proof of Theorem 4.18. Let $\phi$ be any one of the two characters of $E^{\times}$satisfying $\phi\left(U_{E}\right)=1$ and $\phi(t)=\chi(t)^{-1}$. Consider the $E^{\times}$-representation $\phi \Xi_{\chi}$ given by $\left(\phi \Xi_{\chi}\right)(e)=\phi(e) \Xi_{\chi}(e)$. By construction, it is trivial on the subgroup $\left\langle t, U_{E}^{m+1}\right\rangle$ of $E^{\times}$, and we consider it as a representation of the finite group $E^{\times} /\left\langle t, U_{E}^{m+1}\right\rangle \cong U_{E} / U_{E}^{m+1} \times\langle u\rangle /\left\langle u^{2}\right\rangle$. Let $\theta$ be a character of $E^{\times}$. Then $\left\langle\theta, \Xi_{\chi}\right\rangle_{E^{\times}}=0$, unless $\theta$ coincides with $\chi$ on $F^{\times} U_{E}^{m+1}$. Assume this holds. Then $\phi \theta$ also factors through a character of $U_{E} / U_{E}^{m+1} \times\langle u\rangle /\left\langle u^{2}\right\rangle$ and its multiplicity in $\Xi_{\chi}$ can be computed as follows:

$$
\left\langle\theta, \Xi_{\chi}\right\rangle_{E^{\times}}=\left\langle\phi \theta, \phi \Xi_{\chi}\right\rangle_{U_{E} / U_{E}^{m+1} \times\langle u\rangle /\left\langle u^{2}\right\rangle}=\frac{1}{2(q-1) q^{m}} \sum_{g \in U_{E} / U_{E}^{m+1} \times\langle u\rangle /\left\langle u^{2}\right\rangle} \theta\left(g^{-1}\right) \operatorname{tr}\left(g ; \phi \Xi_{\chi}\right) .
$$

Let $\lambda(\theta) \in\{0,1\}$ be such that $\theta(u)=(-1)^{\lambda(\theta)} \chi(u)$ and let $\operatorname{sgn}(\chi)$ be 0 if $\chi$ is even, and 1 otherwise. We deduce from the above and from Proposition 4.22 ;

$$
\left\langle\theta, \Xi_{\chi}\right\rangle_{E^{\times}}=\frac{1}{2}\left(\left\langle\theta, \Xi_{\chi}\right\rangle_{U_{E} / U_{E}^{m+1}}+(-1)^{\lambda(\theta)}\left\langle\theta, \chi+(-1)^{\operatorname{sgn}(\chi)} \chi^{\tau}\right\rangle_{U_{E} / U_{E}^{m+1}}\right) .
$$

Now Theorem 4.18 follows from Proposition 4.19 by a simple case-by-case study.

Proposition 4.22. Let $g \in E^{\times}$with $v_{u}(g)=1$. Then

$$
\operatorname{tr}\left(g ; \Xi_{\chi}\right)=\chi(g)+\chi^{\tau}(g) .
$$

Proof. The proof is given in Section 5.5 .

Corollary 4.23. The character $\chi$ can be reconstructed from the $E^{\times}$-representation $\left.\Xi_{\chi}\right|_{E^{\times}}$.

Proof. By Lemma 4.4. $\left.\Xi_{\chi}\right|_{E^{\times}}$determines $\left.\chi\right|_{F^{\times} U_{E}^{m+1}}$ uniquely. Consider the map

$$
f: A:=\left\{\theta \in\left(E^{\times}\right)^{\vee}:\left.\theta\right|_{F^{\times} U_{E}^{m+1}}=\left.\chi\right|_{F^{\times} U_{E}^{m+1}}\right\} \rightarrow\left\{\theta^{\prime} \in U_{E}^{\vee}:\left.\theta^{\prime}\right|_{U_{F} U_{E}^{m+1}}=\left.\chi\right|_{U_{F} U_{E}^{m+1}}\right\},
$$

given by restricting characters of $E^{\times}$to $U_{E}$. It is surjective and 2-to-1. By Proposition 4.19 and Theorem 4.18, $\chi$ and $\chi^{\tau}$ are the two unique elements among all elements $\theta \in A$, with the following property: $\theta$ occurs in $\Xi_{\chi}$, but the unique element of $f^{-1}(f(\theta)) \backslash\{\theta\}$ does not occur in $\Xi_{\chi}$.

\subsection{Relation to strata, cuspidality.}

Using the unipotent traces computed in Section 4.3, we show the first part of Theorem 4.2 . We use the terminology of intertwining and strata from $[\overline{\mathrm{BH}} \mid \S 11$ and Chapter 4 . The following is analogous to Iv2 Proposition 4.22 and Corollary 4.23. Recall the notation $N_{n}, N_{n}^{n}$ from 
Section 4.3. Let $N$ resp. $N^{n}$ denote the preimage of $N_{n}$ resp. $N_{n}^{n}$ under the natural projection $U_{\mathfrak{J}} \rightarrow U_{\mathfrak{J}} / U_{\mathfrak{J}}^{m+1}$.

Proposition 4.24. Let $m \geqslant 0$. Let $\Xi$ be an irreducible $E^{\times} U_{\mathfrak{J}}$-representation, which is trivial on $U_{\mathfrak{J}}^{m+1}$ and does not contain the trivial character on $N^{n}$. Then the $G(F)$-representation $\Pi_{\Xi}=\mathrm{c}-\operatorname{Ind}_{E^{\times} U_{\mathfrak{J}}}^{G(F)} \Xi$ is irreducible, cuspidal and admissible. Moreover, it contains a ramified simple stratum $(\mathfrak{J}, m, \alpha)$ for some $\alpha \in \varpi^{-m} \mathfrak{J}$. One has $\ell\left(\Pi_{\Xi}\right)=\frac{m}{2}$. For any character $\phi$ of $F^{\times}$ one has $0<\ell\left(\Pi_{\Xi}\right) \leqslant \ell\left(\phi \Pi_{\Xi}\right)$.

From this we can deduce the first statement of Theorem 4.2 ,

Corollary 4.25. Let $(E / F, \chi)$ be a minimal pair. The representation $R_{\chi}$ is irreducible, cuspidal and admissible. It contains a ramified simple stratum and is, in particular, ramified. Moreover, $\ell\left(R_{\chi}\right)=\frac{\ell(\chi)}{2}$ and for any character $\phi$ of $F^{\times}$one has $0<\ell\left(R_{\chi}\right) \leqslant \ell\left(\phi R_{\chi}\right)$.

Proof. The assumptions of Proposition 4.24 are satisfied for the $E^{\times} U_{\mathfrak{J}}$-representation $\Xi_{\chi}$ by Corollary 4.7 and Proposition 4.5 .

Proof of Proposition 4.24. Irreducibility and cuspidality of $\Xi$ follow from $[\overline{\mathrm{BH}}]$ Theorem 11.4, which assumptions are satisfied due to Lemma 4.26. To contain a stratum is defined with respect to an additive character. So fix some character $\psi$ of $F$ of level 1. Make the isomorphism (4.1) explicit for $k=m-1, r=m$ :

$$
\varpi^{-m} \mathfrak{J} / \varpi^{1-m} \mathfrak{J} \stackrel{\sim}{\longrightarrow}\left(U_{\mathfrak{J}}^{m} / U_{\mathfrak{J}}^{m+1}\right)^{\vee}
$$

An element of $\varpi^{-m} \mathfrak{J} / \varpi^{1-m} \mathfrak{J}$ resp. of $U_{\mathfrak{J}}^{m} / U_{\mathfrak{J}}^{m+1}$ is represented by a matrix $a=\left(\begin{array}{cc}a_{2} t^{-n} \\ a_{3} t^{1-n}\end{array}\right)$ resp. $x=\left(\begin{array}{cc}x_{2} t^{n-1} \\ x^{n}\end{array}\right)$ with $a_{2}, a_{3}, x_{2}, x_{3} \in k$ and $\psi_{\mathfrak{M}, a}(x)=\psi\left(a_{2} x_{3}+a_{3} x_{2}\right)$. The restriction of $\Xi$ to $U_{\mathfrak{J}}^{m}$ factors through a representation of the abelian group $U_{\mathfrak{J}}^{m} / U_{\mathfrak{J}}^{m+1}$, thus it decomposes as a sum of characters, each of which is of the form $\left.\psi_{\mathfrak{M}, a}\right|_{U_{\mathfrak{J}}^{m}}$ for some $a \in \varpi^{-m} \mathfrak{J}$. With other words, for each $a$, such that $\left.\psi_{\mathfrak{M}, a}\right|_{U_{\mathfrak{J}}^{m}}$ is contained in $\Xi$, the ramified stratum $(\mathfrak{J}, m, a)$ occurs in $\Pi_{\Xi}$. By definition, a ramified stratum is simple, if and only it is fundamental, i.e., the coset $a+\varpi^{1-m} \mathfrak{J}$ does not contain a nilpotent element of $\mathfrak{M}$. Thus to show that $\Pi_{\Xi}$ contains a ramified simple stratum it is enough to show the following claim.

Claim. Let $a \in \varpi^{-m} \mathfrak{J}$. Assume $\left.\psi_{\mathfrak{M}, a}\right|_{U_{\mathfrak{J}}^{m}}$ occurs in $\Xi$. Then $a+\varpi^{1-m} \mathfrak{J}$ does not contain nilpotent elements of $\mathfrak{M}$, or with other words $a_{2}, a_{3} \neq 0$ (with notations as above).

Proof of the claim. Assume $a_{2}=0$, then the restriction of $\psi_{\mathfrak{M}, a}$ to the subgroup $N^{n}$ of $U_{\mathfrak{J}}^{m}$ is the trivial character, which contradicts our assumptions on $\Xi$. Thus $a_{2} \neq 0$. Assume $a_{3}=0$. As $\varpi \in E^{\times} U_{\mathfrak{J}}$, the character $\psi_{\varpi a \varpi^{-1}}$ also occurs in $\Xi$ (proof as in Iv2 Lemma 4.25). But $\varpi a \varpi^{-1}=\left(\begin{array}{cc}a_{2} t^{1-n} & a_{3} t^{-n}\end{array}\right)$ and we deduce a contradiction as in the already proven part.

Thus we have shown that $\Pi_{\Xi}$ contains a ramified fundamental stratum of the form $(\mathfrak{J}, m, a)$. Then $\left[\mathrm{BH}\right.$ Theorem 12.9 shows that $\ell\left(\Pi_{\Xi}\right)=\frac{m}{2}$. Furthermore, if an essentially scalar stratum would be contained in $\Pi_{\Xi}$, then by $[\mathrm{BH}]$ Section 12.9, it would have to intertwine with $(\mathfrak{J}, m, a)$. But by [BH] 13.2 Proposition, no fundamental stratum of the form $(\mathfrak{M}, r, b)$ can intertwine with the fundamental ramified stratum $(\mathfrak{J}, m, a)$. Thus no essentially scalar stratum is contained in $\Pi_{\Xi}$ and $[\mathrm{BH}] 13.3$ Theorem shows the last statement of the proposition.

Lemma 4.26. Let $\Xi$ be an irreducible $E^{\times} U_{\mathfrak{J}}$-representation, which is trivial on $U_{\mathfrak{J}}^{m+1}$ and does not contain the trivial character on $N^{n}$. An element $g \in G(F)$ intertwines $\Xi$ if and only if $g \in E^{\times} U_{\mathfrak{J}}$. 
Proof. The double $E^{\times} U_{\mathfrak{J}^{-}}$cosets in $G(F)$ are represented by diagonal matrices with entries $t^{\alpha}, 1$ for $\alpha \geqslant 0$. The rest of the proof works exactly as in $[\overline{\mathrm{Iv} 2}]$ Lemma 4.24.

\subsection{Relation to cuspidal inducing data.}

We relate the representations $R_{\chi}, \pi_{\chi}$ to each other. The following proposition finishes the proof of Theorem 4.2 .

Proposition 4.27. Let $(E / F, \chi)$ be an admissible pair. Then $R_{\chi} \cong \pi_{\chi}$.

Proof. By twisting both sides with a character of $F^{\times}$, we can assume that $(E / F, \chi)$ is a minimal pair. By construction of $\pi_{\chi}$ and Lemma 4.3 , it is enough to show that $\Xi_{\chi} \cong \Theta_{\chi}\left(\Theta_{\chi}\right.$ is as in Section 4.1.6). From Corollary 4.25 and the proof of Proposition 4.24 it follows that there is a simple (ramified) stratum $(\mathfrak{J}, m, \beta)$ such that $\left.\Xi_{\chi}\right|_{U_{\mathfrak{J}}^{m}}$ contains $\psi_{\beta}$. By $\mid \mathrm{BH} 15.8$ Exercise it follows that $\left(E^{\times} U_{\mathfrak{J}}, \Xi_{\chi}\right)$ is a cuspidal inducing datum in $G(F)$, i.e., there is some $\chi^{\prime}$ with $\Xi_{\chi} \cong \Theta_{\chi^{\prime}}$. By the last statement of Corollary 4.25 . $\left(E / F, \chi^{\prime}\right)$ has to be minimal. By Lemma 4.28, $\left.\left.\Theta_{\chi^{\prime}}\right|_{E^{\times}} \cong \Xi_{\chi^{\prime}}\right|_{E^{\times}}$. Thus $\left.\left.\Xi_{\chi^{\prime}}\right|_{E^{\times}} \cong \Xi_{\chi}\right|_{E^{\times}}$. Now, by Corollary 4.23, $\chi$ is (up to $\tau$-conjugacy) uniquely determined by $\Xi_{\chi}$, and we deduce $\chi^{\prime}=\chi$ or $\chi^{\prime}=\chi^{\tau}$. As $\Theta_{\chi} \cong \Theta_{\chi^{\tau}}$, the proposition follows.

Lemma 4.28. Let $(E / F, \chi)$ be a minimal pair. We have $\left.\left.\Theta_{\chi}\right|_{E^{\times}} \cong \Xi_{\chi}\right|_{E^{\times}}$.

Proof. The proof is given in Section 5.6 .

\subsection{Small level case.}

Let $\chi$ be a character of $E^{\times}$of (odd) level $m \geqslant 1$. Let $n \geqslant m+1$ be an integer. Then $\chi$ defines a character $\tilde{\chi}$ of the group $E^{\times} U_{\mathfrak{J}}^{n}$ by composition

$$
\tilde{\chi}: E^{\times} U_{\mathfrak{J}}^{n} \rightarrow E^{\times} U_{\mathfrak{J}}^{n} / U_{\mathfrak{J}}^{n} \cong E^{\times} / U_{E}^{n} \rightarrow E^{\times} / U_{E}^{m+1} \stackrel{\chi}{\rightarrow} \overline{\mathbb{Q}}_{\ell}^{\times} .
$$

Proposition 4.29. Let $\chi$ be a character of $E^{\times}$of odd level $m \geqslant 1$ and let $n \geqslant m+1$. Then

$$
\mathrm{R}_{\chi, n} \cong \mathrm{c}-\operatorname{Ind}_{E^{\times} U_{\mathfrak{J}}^{n}}^{G(F)} \tilde{\chi} .
$$

Proof of Proposition 4.29. By Lemma 4.3 it is enough to show $\Xi_{\chi, n} \cong \mathrm{c}-\operatorname{Ind}_{E^{\times} U_{E}^{n}}^{E^{\times}} \tilde{U_{\mathfrak{X}}}$. To do so, it is enough to show that the traces of each element of $E^{\times} U_{\mathfrak{J}}$ in both spaces agree. Modulo center, which acts by $\left.\chi\right|_{F^{\times}}$in both spaces, any element of $E^{\times} U_{\mathfrak{J}}$ is represented by an element in $U_{\mathfrak{J}} \cup \varpi U_{\mathfrak{J}}$, thus we can restrict to elements lying in this union. The required trace computations are covered by Lemmas 4.30 and 4.31 .

Lemma 4.30. Let $g \in U_{\mathfrak{J}}$. Precisely one of the following cases occurs:

(i) $g \in U_{F} U_{\mathfrak{J}}^{n}$. Then $\left.\operatorname{tr}\left(g ; \Xi_{\chi, n}\right)[\chi]\right)=(q-1) q^{n-1} \tilde{\chi}(g)$. In particular, $U_{\mathfrak{J}}^{n}$ acts trivial in $\left.\Xi_{\chi, n}\right)[\chi]$ and $U_{F}$ acts through the character $\left.\chi\right|_{F^{\times}}$.

(ii) $g \in U_{\mathfrak{J}} \backslash U_{F} U_{\mathfrak{J}}^{n}$ is conjugate to an element $x$ of $U_{F} U_{E}^{2 \alpha+1} U_{\mathfrak{J}}^{n} \backslash U_{F} U_{E}^{2 \alpha+3} U_{\mathfrak{J}}^{n}$, such that $2 \alpha+2 \leqslant n$. Then

$$
\left.\operatorname{tr}\left(g ; \Xi_{\chi, n}\right)[\chi]\right)=q^{2 \alpha+1}\left(\tilde{\chi}(x)+\tilde{\chi}^{\tau}(x)\right) .
$$

(iii) $g \in U_{\mathfrak{J}} \backslash U_{F} U_{\mathfrak{J}}^{n}$ is not conjugate to an element of $U_{E} U_{\mathfrak{J}}^{n}$. Then $\left.\operatorname{tr}\left(g ; \Xi_{\chi, n}\right)[\chi]\right)=0$.

Let $g \in \varpi U_{\mathfrak{J}}$. Precisely one of the following two cases can occur:

(i) $g$ is not conjugate to an element of $E^{\times} U_{\mathfrak{J}}^{n}$. Then $\left.\operatorname{tr}\left(g ; \Xi_{\chi, n}\right)[\chi]\right)=0$.

(ii) $^{\prime} g$ is conjugate to an element $x$ of $E^{\times} U_{\mathfrak{J}}^{n}$. Then $\left.\operatorname{tr}\left(g ; \Xi_{\chi, n}\right)[\chi]\right)=\tilde{\chi}(x)+\tilde{\chi}^{\tau}(x)$.

Proof. The proof is given in Section 5.7 . 
Lemma 4.31. Lemma 4.30 holds with $\Xi_{\chi, n}$ replaced by $\mathrm{c}-\operatorname{Ind}_{E^{\times} U_{E}^{n}}^{E^{\times} U_{\mathfrak{X}}} \tilde{\text {. }}$

Proof. The lemma follows by an explicit computation using the Mackey-formula in a way very similar to the proof of Lemma 4.28. We omit the details.

Using notations from Definition 4.16 we have the following structure result.

Theorem 4.32. Let $\chi$ be a character of $E^{\times}$of odd level $m \geqslant 1$ and let $n \geqslant m+1$. Let $\theta$ be a character of level $\geqslant m$. There are no non-zero maps from $R_{\chi, n}$ to $R_{\theta}$, unless $\theta$ coincides with $\chi$ on $F^{\times} U_{E}^{m+1}$. In this case, we have

$\operatorname{Hom}_{G(F)}\left(R_{\chi, n}, R_{\theta}\right)= \begin{cases}\overline{\mathbb{Q}}_{\ell} & \text { if } \theta=\chi, \text { or } \theta=\chi^{\tau}, \text { or the distance from } \theta \text { to } \chi \text { is properly quadratic } \\ 0 & \text { otherwise. }\end{cases}$ In particular, the character $\chi$ can be reconstructed from $R_{\chi, n}$.

Proof. By Lemma 4.33 we may assume that $\theta$ and $\chi$ coincide on $F^{\times} U_{\mathfrak{J}}^{m+1}$. Thus by our assumption on $\theta,(E / F, \chi)$ is a minimal pair and we compute

$$
\begin{aligned}
\operatorname{Hom}_{G(F)}\left(R_{\chi, n}, R_{\theta}\right) & =\operatorname{Hom}_{E^{\times} U_{\mathfrak{J}}}\left(\Xi_{\chi, n}, \Xi_{\theta}\right) \\
& =\operatorname{Hom}_{E^{\times} U_{\mathfrak{J}}^{n}}\left(\tilde{\chi}, \Xi_{\theta}\right) \\
& =\operatorname{Hom}_{E^{\times}}\left(\tilde{\chi}, \Xi_{\theta}\right) \\
& =\operatorname{Hom}_{E^{\times}}\left(\tilde{\chi}, \bigoplus_{\theta^{\prime}} \theta^{\prime}\right),
\end{aligned}
$$

where $\theta^{\prime}$ runs through the set of all characters of $E^{\times}$coinciding with $\theta$ on $F^{\times} U_{E}^{m+1}$, such that either $\theta^{\prime}=\theta$, or $\theta^{\prime}=\theta^{\tau}$, or the distance from $\theta$ to $\theta^{\prime}$ is properly quadratic. Above, the first equality follows from Lemma 4.33. The second is Frobenius reciprocity and Proposition 4.29. The third follows, as $n \geqslant m+1$ and hence $\tilde{\chi}, \Xi_{\theta}$ are trivial on $U_{\mathfrak{J}}^{n}$. Finally, the forth equality follows from Theorem 4.18. The above computation shows the statement of the theorem about $\operatorname{Hom}_{G(F)}\left(R_{\chi, n}, R_{\theta}\right)$. It remains to show that $\chi$ can be reconstructed from $R_{\chi, n}$. First, the above considerations characterize $m$ as the greatest odd integer, such that there are non-zero maps from $R_{\chi, n}$ to $R_{\theta}$ for some $\theta$ of level $m$. The rest follows as in the proof of Corollary 4.23 .

Lemma 4.33. Let $\theta$ be a character of $E^{\times}$of odd level $\ell(\theta) \geqslant m$. If $\theta$ does not coincide with $\chi$ on $F^{\times} U_{E}^{m+1}$, then there are no non-zero morphisms between $R_{\chi, n}$ and $R_{\theta}$. Assume $\theta$ coincides with $\chi$ on $F^{\times} U_{E}^{m+1}$ (in particular, $\ell(\theta)=m$ ). Then

$$
\operatorname{Hom}_{G(F)}\left(R_{\chi, n}, R_{\theta}\right)=\operatorname{Hom}_{E^{\times} U_{\mathfrak{J}}}\left(\Xi_{\chi, n}, \Xi_{\theta}\right) .
$$

Proof. Applying twice the Frobenius reciprocity and once the Mackey formula, we see by Lemma 4.3

$$
\operatorname{Hom}_{G(F)}\left(R_{\chi, n}, R_{\theta}\right)=\bigoplus_{g \in E^{\times} U_{\mathfrak{J}} \backslash G(F) / E^{\times} U_{\mathfrak{J}}} \operatorname{Hom}_{E^{\times} U_{\mathfrak{J}} \cap^{g}\left(E^{\times} U_{\mathfrak{J}}\right)}\left({ }^{g} \Xi_{\chi, n}, \Xi_{\theta}\right),
$$

where ${ }^{g}\left(E^{\times} U_{\mathfrak{J}}\right)=g\left(E^{\times} U_{\mathfrak{J}}\right) g^{-1}$ and ${ }^{g} \Xi_{\chi, n}(x)=\Xi_{\chi, n}\left(g^{-1} x g\right)$. The set $E^{\times} U_{\mathfrak{J}} \backslash G(F) / E^{\times} U_{\mathfrak{J}}$ is represented by the diagonal matrices $e_{0}\left(t^{\alpha}, 1\right)$ for $\alpha \geqslant 0$. Let $g=e_{0}\left(t^{\alpha}, 1\right)$ with $\alpha>0$. We show that the summand on the right side corresponding to $g$ vanish. Note that $E^{\times} U_{\mathfrak{J}} \cap{ }^{g}\left(E^{\times} U_{\mathfrak{J}}\right) \supseteq$ $e_{-}\left(\mathfrak{p}_{F}^{\frac{m+1}{2}}\right)$. On the one hand, Proposition 4.5 shows that $\Xi_{\theta}$ does not contain the trivial character on $e_{-}\left(\mathfrak{p}_{F}^{\frac{m+1}{2}}\right)$. On the other hand, $e_{-}\left(\mathfrak{p}_{F}^{\frac{m+1}{2}+\alpha}\right) \subseteq U_{\mathfrak{J}}^{m+1}$ as $\alpha>0$. As $U_{\mathfrak{J}}^{m+1}$ is normal in $E^{\times} U_{\mathfrak{J}}$, and $\tilde{\chi}$ is trivial on $U_{\mathfrak{J}}^{m+1}$, we see by Proposition 4.29 that $\Xi_{\chi, n}$ is trivial on $U_{\mathfrak{J}}^{m+1}$ and, in particular, on $e_{-}\left(\mathfrak{p}_{F}^{\frac{m+1}{2}+\alpha}\right)$. As 


$$
{ }^{g} \Xi_{\chi, n}\left(e_{-}(x)\right)=\Xi_{\chi, n}\left(e_{-}\left(t^{\alpha} x\right)\right),
$$

we deduce that ${ }^{g} \Xi_{\chi, n}$ is trivial on $e_{-}\left(\mathfrak{p}_{F}^{\frac{m+1}{2}}\right)$. The claim follows, and hence

$$
\operatorname{Hom}_{G(F)}\left(R_{\chi, n}, R_{\theta}\right)=\operatorname{Hom}_{E^{\times} U_{\mathfrak{J}}}\left(\Xi_{\chi, n}, \Xi_{\theta}\right) .
$$

It remains to show that this space is 0 , unless $\theta$ coincides with $\chi$ on $F^{\times} U_{E}^{m+1}$. Asssume $\operatorname{Hom}_{E^{\times} U_{\mathfrak{J}}}\left(\Xi_{\chi, n}, \Xi_{\theta}\right) \neq 0$. By comparing central characters, we see that $\left.\theta\right|_{F^{\times}}=\left.\chi\right|_{F^{\times}}$. As above, we see that $\Xi_{\chi, n}$ is trivial on $U_{\mathfrak{J}}^{m+1}$. Hence $\Xi_{\theta}$ is too. We deduce $\ell(\theta)=m$. This shows our claim.

\section{Trace computations}

In this section we use notations from Sections 3 and 4 , especially from Notations $3.1,3.4$ and Definition 3.6 For $g \in G(F)$ we always write $g=\left(\begin{array}{ll}g_{1} & g_{2} \\ g_{3} & g_{4}\end{array}\right)$.

\subsection{Left and right group actions on $X_{\underline{w}_{m}}^{m}(1)$.}

To apply a trace formula in what follows, we make here the actions 3.10 explicit using the coordinates $\psi_{\dot{v}}^{m}$ from 3.2 . It is clear that $I$ acts on $C_{v}^{m} \subseteq \mathscr{F}^{m}$ by left multiplication. The following proposition describes this action.

Proposition 5.1. Let $\dot{v}, v, n$ be as in Notation 3.1 and $m \geqslant 1$ odd (we do not assume $2 n-1 \geqslant m$ here). Let $\dot{x} I^{m}=\psi_{\dot{v}}^{m}(a, C, D, A, B)$ be a point of $C_{v}^{m}$. Then $g \in I$ acts on $\dot{x} I^{m}$ by

$$
\begin{aligned}
g . \dot{x} I^{m}= & \psi_{\dot{v}}^{m}\left(\left.g \cdot a\right|_{n}, \frac{\operatorname{det}(g)}{g_{2} a+g_{1}} C N^{-1},\left(g_{2} a+g_{1}\right) D N, \ldots\right. \\
& \left.\ldots A N+h(g, a) \frac{\left(g_{2} a+g_{1}\right)^{2} D N^{2}}{\operatorname{det}(g) C}, B+u^{n+1} \frac{g_{2}}{\left(g_{2} a+g_{1}\right)} C D^{-1} N^{-1}\right),
\end{aligned}
$$

where

$$
\begin{aligned}
g . a & :=\frac{g_{4} a+g_{3}}{g_{2} a+g_{1}} \in L_{[1, n+m]} \mathbb{G}_{a}(\bar{k}) \quad \text { and }\left.\cdot\right|_{n} \text { is as in Section 3.1.5 } \\
N & :=1+u^{n+1} \frac{g_{2}}{g_{2} a+g_{1}} C D^{-1} A \\
h(g, a) & :=u^{-(n+1)}\left(\text { g.a-g.a }\left.\right|_{n}\right) \in L_{[0, m-1]} \mathbb{G}_{a}(\bar{k}) .
\end{aligned}
$$

Proof. First, observe that the expressions in the proposition are well-defined, as $v_{u}\left(g_{1}\right)=$ $v_{u}\left(g_{4}\right)=0, v_{u}\left(g_{2}\right) \geqslant 0, v_{u}\left(g_{3}\right)>0$ and $v_{u}(a)>0$. We compute in $G(\breve{E})$ (with $a, g . a, C, D$ replaced by some representatives in $\bar{k} \llbracket u \rrbracket)$ :

$$
g e_{-}(a) \dot{v} e_{0}(C, D)=e_{-}(g . a) \dot{v} e_{0}\left(\frac{\operatorname{det}(g) C}{g_{2} a+g_{1}},\left(g_{2} a+g_{1}\right) D\right) e_{-}\left(u^{n+1} \frac{g_{2} C D^{-1}}{g_{2} a+g_{1}}\right) .
$$

Further, using (3.4) we see that

$$
e_{-}\left(u^{n+1} \frac{g_{2} C D^{-1}}{g_{2} a+g_{1}}\right) e_{+}(A) e_{-}(B)=e_{0}\left(N^{-1}, N\right) e_{+}(A N) e_{-}\left(B+u^{n+1} \frac{g_{2} C D^{-1}}{g_{2} a+g_{1}} N^{-1}\right),
$$

with $N$ as in the proposition. Combining the two last computations we see: 


$$
\begin{aligned}
g . \dot{x} I^{m} & =g e_{-}(a) \dot{v} e_{0}(C, D) e_{+}(A) e_{-}(B) \\
& =e_{-}(g \cdot a) \dot{v} e_{0}\left(\frac{\operatorname{det}(g) C}{g_{2} a+g_{1}},\left(g_{2} a+g_{1}\right) D\right) e_{-}\left(u^{n+1} \frac{g_{2} C D^{-1}}{g_{2} a+g_{1}}\right) e_{+}(A) e_{-}(B) \\
& =e_{-}(g . a) \dot{v} e_{0}\left(\frac{\operatorname{det}(g) C}{g_{2} a+g_{1}},\left(g_{2} a+g_{1}\right) D\right) e_{0}\left(N^{-1}, N\right) e_{+}(A N) e_{-}\left(B+u^{n+1} \frac{g_{2} C D^{-1}}{g_{2} a+g_{1}}\right) .
\end{aligned}
$$

Now the only thing we have to do, is to replace $g . a \in L_{[1, n+m]} \mathbb{G}_{a}(\bar{k})$ in the last expression by an element in $L_{[1, n+m]}^{\leqslant n} \mathbb{G}_{a}(\bar{k})$. Therefore, note that $g \cdot a$ and $\left.g \cdot a\right|_{n}$ have the same image in $L_{[1, n]} \mathbb{G}_{a}(\bar{k})$, i.e., g.a $-\left.g \cdot a\right|_{n}$ is divisible by $u^{n+1}$ and $h(g, a)$ is well-defined as an element of $L_{[0, m-1]} \mathbb{G}_{a}(\bar{k})$ and that

$$
e_{-}(g . a) \dot{v}=e_{-}\left(g .\left.a\right|_{n}\right) e_{-}\left(g . a-g .\left.a\right|_{n}\right) \dot{v}=e_{-}\left(g .\left.a\right|_{n}\right) \dot{v} e_{+}(h(g, a)) .
$$

Combining this and (5.1) finishes the proof of the proposition.

We compute $h(g, a)$ from Proposition 5.1 in some cases of interest for us. We point out, that later we need to know $h(g, a)$ only modulo $u^{n}$ (cf. Proposition 5.6).

Lemma 5.2. Let $n, m$ be as in Proposition 5.1. Assume $m \leqslant 2 n-1$. Let $g \in U_{\mathfrak{J}}$ and $a \in$ $L_{[1, n+m]}^{\leqslant n}(k)$ with $a_{1} \neq 0$.

(i) For $g \in U_{\mathfrak{J}}^{m+1}$ we have $v_{u}(h(g, a)) \geqslant n$.

Let $g=\iota\left(1+y u^{2 \alpha+1}\right)$ with $0 \leqslant \alpha \leqslant n-1$ and $y \in U_{F}$. Write $a=u a^{\prime}$

(ii) If $\alpha \geqslant\left\lfloor\frac{n}{2}\right\rfloor$, then $h(g, a)=u^{2 \alpha+1-n} y\left(1-a^{\prime 2}\right)\left(1-u^{2 \alpha+1} y a^{\prime}\right)$.

(iii) If $0 \leqslant \alpha<\left\lfloor\frac{n}{2}\right\rfloor$ and $a^{\prime}= \pm 1+u^{n-2 \alpha-1} b$ for some $b \in L_{[0 ; m+2 \alpha+1]}^{\leqslant 2 \alpha} \mathbb{G}_{a}(k)$, then $h(g, a)=$ $\frac{y\left(\mp 2 b-u^{n-2 \alpha-1} b^{2}\right)}{1 \pm u^{2 \alpha+1} y+u^{n} y b}$.

Proof. In any of the three cases, a simple calculation shows $g .\left.a\right|_{n}=a$ (only this case is of interest for us, cf. (5.2)). Now (i) is an easy computation. For (ii) and (iii) we compute

$$
u^{n+1} h(g, a)=g . a-\left.g \cdot a\right|_{n}=g \cdot a-a=\frac{a+u^{2 \alpha+2} y}{1+u^{2 \alpha} y a}-a=\frac{u^{2 \alpha+2} y\left(1-a^{\prime, 2}\right)}{1+u^{2 \alpha+1} y a^{\prime}} .
$$

From this the lemma follows.

Let $\varpi$ be as in Section 3.1 .3 and $y_{1}:=e_{0}(u,-u)$. Left multiplication by $\varpi$ composed with right multiplication by $y_{1}^{-1}$ defines an automorphism $\tilde{\beta}_{\varpi}$ of $\tilde{Y}_{\dot{w}}^{m}$. By (the proof of) Lemma 3.11 . $\tilde{\beta}_{\varpi}$ restricts to an automorphism

$$
\beta_{\varpi}: Y_{\dot{w}}^{m} \stackrel{\sim}{\rightarrow} Y_{\dot{w}}^{m} \quad \text { given by } \quad \dot{x} I^{m} \mapsto \varpi \dot{x} y_{1}^{-1} I^{m} .
$$

Proposition 5.3. Let $\dot{v}, v, n, D_{w}^{\tau}$ be as in Notation 3.1 and $2 n-1 \geqslant m \geqslant 1$ odd. Let $\psi_{\dot{v}}^{m}( \pm u, C, D, A, B)$ be a point of $Y_{\dot{w}}^{m}$ lying over $\pm u \in D_{w}^{\tau}$. Then

$$
\beta_{\varpi}\left(\psi_{\dot{v}}^{m}( \pm u, C, D, A, B)\right)=\psi_{\dot{v}}^{m}\left( \pm u, \mp C M^{-1}, \mp D M,-A M, B\right),
$$

where $M:=1-2 u^{n} C \tau(C)^{-1} A$.

Proof. Write $\dot{x} I^{m}=\psi_{\dot{v}}^{m}( \pm u, C, D, A, B)$. Using formulas (3.4) we compute

$$
\begin{aligned}
\beta_{\varpi}\left(\dot{x} I^{m}\right) & =e_{-}( \pm u) \dot{v} e_{0}(\mp 1, \mp 1) e_{-}\left(\mp u^{n}\right) e_{0}(C, D) e_{+}(-A) e_{-}(-B) I^{m}= \\
& =e_{-}( \pm u) \dot{v} e_{0}\left(\mp C M^{\prime,-1}, \mp D M^{\prime}\right) e_{+}\left(-A M^{\prime}\right) e_{-}\left(\mp u^{n} C D^{-1}-B\right),
\end{aligned}
$$


where $M^{\prime}:=1 \pm u^{n} C D^{-1} A$. Now $a= \pm u$ gives $R=u^{-1}(\tau(a)-a)=u^{-1}(\mp u-( \pm u))=\mp 2$ and as $\dot{x} I^{m} \in Y_{\dot{w}}^{m}$, we have $D^{-1} \equiv R \tau(C)^{-1} \equiv \mp 2 \tau(C)^{-1} \bmod u^{n}$ and $B=u^{n} C \tau(C)^{-1}$. This shows on the one hand $M^{\prime}=M$, and on the other hand $\mp u^{n} C D^{-1}-B=2 B-B=B$.

Now we make the right $I_{m, \underline{w}_{m}} / I^{m}$-action on $Y_{\dot{w}}^{m}$ explicit.

Proposition 5.4. Let $\dot{v}, v, n$ be as in Notation 3.1 and $m \geqslant 1$ odd (we do not assume $2 n-1 \geqslant m$ here). Let $\psi_{\dot{v}}^{m}(a, C, D, A, B)$ be a point of $C_{v}^{m}$. Then $i=\left(\begin{array}{cc}i_{1} & \\ & \tau\left(i_{1}\right)\end{array}\right)\left(\begin{array}{cc}1 & i_{2} \\ & 1\end{array}\right) \in I_{m, \underline{w}_{m}} / I^{m}$ acts on $\psi_{\dot{v}}^{m}(a, C, D, A, B)$ by

$$
\psi_{\dot{v}}^{m}(a, C, D, A, B) . i=\psi_{\dot{v}}^{m}\left(a, C i_{1} H^{-1}, D \tau\left(i_{1}\right) H, i_{1}^{-1} \tau\left(i_{1}\right) H^{2} A+i_{2} H, i_{1} \tau\left(i_{1}\right)^{-1} B H^{-1}\right),
$$

where $H=1+i_{1} \tau\left(i_{1}\right)^{-1} i_{2} B \in \bar{k}[u] / u^{m+1}$ (note that $i_{2}$ is only determined $\bmod u^{m}$, but $B \equiv 0$ $\bmod u$ ).

Proof. The proof is a computation similar to (and simpler as) the proof of Proposition 5.1 .

\subsection{Generalities on the trace formula.}

We use the following trace formula due to Boyarchenko.

Lemma 5.5 ( $[\mathrm{BO}]$ Lemma 2.12). Let $X$ be a separated scheme of finite type over a finite field $\mathbb{F}_{Q}$ with $Q$ elements, on which a finite group $A$ acts on the right. Let $g: X \rightarrow X$ be an automorphism of $X$, which commutes with the action of $A$. Let $\psi: A \rightarrow \overline{\mathbb{Q}}_{\ell}^{*}$ be a character of $A$. Assume that $\mathrm{H}_{c}^{i}(X)[\psi]=0$ for $i \neq i_{0}$ and $\operatorname{Frob}_{Q}$ acts on $\mathrm{H}_{c}^{i_{0}}(X)[\psi]$ by a scalar $\lambda \in \overline{\mathbb{Q}}_{\ell}^{*}$. Then

$$
\operatorname{Tr}\left(g^{*}, \mathrm{H}_{c}^{i_{0}}(X)[\psi]\right)=\frac{(-1)^{i_{0}}}{\lambda \cdot \sharp A} \sum_{a \in A} \psi(a) \cdot \sharp S_{g, a},
$$

where $S_{g, a}=\left\{x \in X\left(\overline{\mathbb{F}_{q}}\right): g\left(\operatorname{Frob}_{Q}(x)\right)=x \cdot a\right\}$.

We adapt Lemma 5.5 to our situation.

Proposition 5.6. Let $n \geqslant 1, m \geqslant 1$ two integers with $m \leqslant 2 n+1$. Let $\chi$ be a character of $E^{\times}$ of level $m$. Let $g \in U_{\mathfrak{J}}$. Then

$$
\operatorname{tr}\left(g ; \mathrm{H}_{c}^{0}\left(\tilde{Y}_{\dot{w}}^{m}\right)[\chi]\right)=\sum_{i_{1} \in U_{E} / U_{E}^{m+1}} \sharp S_{g, i_{1}} \chi\left(i_{1}\right),
$$

where $S_{g, i_{1}}$ is empty, unless $\operatorname{det}(g) \equiv i_{1} \tau\left(i_{1}\right) \bmod u^{m+1}$, in which case it is the set of solutions of the equations

$$
\begin{aligned}
g_{2} a^{2}+\left(g_{1}-g_{4}\right) a-g_{3} & \equiv 0 \quad \bmod u^{n+1} \\
\tau\left(i_{1}\right)\left(1+u^{n} h(g, a) R^{-1}\right) & \equiv g_{2} a+g_{1} \quad \bmod u^{m+1}
\end{aligned}
$$

in $a \in L_{[1, n+m]}^{\leq n} \mathbb{G}_{a}(k)$ (with $a_{1} \neq 0$ ), where

$$
\begin{aligned}
h(g, a) & =u^{-(n+1)}\left(g \cdot a-\left.g \cdot a\right|_{u^{n}}\right) \in L_{[0, m-1]} \mathbb{G}_{a}(\bar{k}) \\
R & =u^{-1}(\tau(a)-a) .
\end{aligned}
$$

Lemma 5.7. Let $\chi$ be a character of $E^{\times}$. We have $\mathrm{H}_{c}^{0}\left(\tilde{Y}_{\dot{w}}^{m}\right)[\chi] \cong \mathrm{H}_{c}^{0}\left(Y_{\dot{w}}^{m}\right)\left[\left.\chi\right|_{U_{E}}\right]$.

Proof. The proof is the same as in [Iv2] Lemma 4.5. 
Lemma 5.8. Let $n \leqslant s \leqslant 2 n$ be positive integers. Let $f \in k[u] /\left(u^{s}\right)$ and let $h: k[u] /\left(u^{n}\right) \rightarrow$ $k[u] /\left(u^{s-n}\right)$ be some map. Then for $x \in k[u] /\left(u^{s}\right)$ we have

$$
x=f+u^{n} h\left(x \bmod u^{n}\right) \quad \Leftrightarrow \quad x=f+u^{n} h\left(f \bmod u^{n}\right)
$$

(both equalities take place in $k[u] /\left(u^{s}\right)$ ).

Proof. This is trivial.

Proof of Proposition 5.6. The action of $g$ on $\tilde{Y}_{\dot{w}}^{m}$ fixes $Y_{\dot{w}}^{m}$. By Lemma 5.7 we have

$$
\operatorname{tr}\left(g ; \mathrm{H}_{c}^{0}\left(\tilde{Y}_{\dot{w}}^{m}\right)[\chi]\right)=\operatorname{tr}\left(g ; \mathrm{H}_{c}^{0}\left(Y_{\dot{w}}^{m}\right)\left[\left.\chi\right|_{U_{E}}\right]\right) .
$$

We have $\sharp I_{m, \underline{w}_{m}} / I^{m}=(q-1) q^{2 m}$. Applying Lemma 5.5 to the left action of $U_{\mathfrak{J}}$ and the right action of $I_{m, \underline{w}_{m}} / I^{m}$ on $Y_{\dot{w}}^{m}$ and Frob $_{q}$ (this is possible, as only the zeroth cohomology is nonvanishing, and as the Frobenius acts as a scalar in $\mathrm{H}_{c}^{0}$ ), we deduce

$$
\operatorname{tr}\left(g ; \mathrm{H}_{c}^{0}\left(\tilde{Y}_{\dot{w}}^{m}\right)[\chi]\right)=\frac{1}{(q-1) q^{2 m}} \sum_{i \in I_{m, \underline{w}_{m}} / I^{m}} \sharp S_{g, i} \chi(i),
$$

where $S_{g, i}$ is the set of points $y \in Y_{\dot{w}}^{m}$ with $g . y=y . i$ (note that any point in $Y_{\dot{w}}^{m}$ has coordinates in $k$, hence Frobenius acts trivial). Further, note that a point of $Y_{\dot{w}}^{m}$ is uniquely determined by its coordinates $a, C, A$ (cf. Definition 3.6. Write $i=\left(\begin{array}{cc}i_{1} & \\ & \tau\left(i_{1}\right)\end{array}\right)\left(\begin{array}{cc}1 & i_{2} \\ & 1\end{array}\right)$ with $i_{1} \in$ $U_{E} / U_{E}^{m+1}, i_{2} \in k[u] / u^{m}$. As the determinant is multiplicative, we see that $S_{g, i}=\varnothing$, unless $\operatorname{det}(g) \equiv \operatorname{det}(i)=i_{1} \tau\left(i_{1}\right) \bmod u^{m+1}$. Assume this holds. By Propositions 5.1 and 5.4 , we see that $\sharp S_{g, i}$ is equal to the number of solutions of the equations

$$
\begin{aligned}
g .\left.a\right|_{n} & \equiv a \bmod u^{n+1} \\
\frac{\operatorname{det}(g)}{g_{2} a+g_{1}} C N^{-1} & \equiv C i_{1} H^{-1} \bmod u^{m+1} \\
A N+h(g, a) \frac{\left(g_{2} a+g_{1}\right)^{2} D N^{2}}{\operatorname{det}(g) C} & \equiv i_{1}^{-1} \tau\left(i_{1}\right) H^{2} A+i_{2} H \bmod u^{m}
\end{aligned}
$$

in the variables $a=\sum_{i=1}^{n} a_{i} u^{i}+\sum_{i=n+1}^{m} 0 u^{i} \in L_{[1, n+m]}^{\leqslant n} \mathbb{G}_{a}(k)$ (with $\left.a_{1} \neq 0\right), C \in\left(k[u] / u^{m+1}\right)^{\times}$ and $A \in k[u] / u^{m}$, where

$$
\begin{aligned}
& B=u^{n} C \tau(C)^{-1} \\
& D=R^{-1} \tau(C)\left(1+u^{n} C \tau(C)^{-1} A-u^{n} C^{-1} \tau(C) \tau(A)\right)
\end{aligned}
$$

(as we are in $Y_{\dot{w}}^{m}$; here $R=u^{-1}(\tau(a)-a)$ ) and $h(g, a)$ and

$$
\begin{aligned}
N & =1+u^{n+1} \frac{g_{2}}{g_{2} a+g_{1}} C D^{-1} A \equiv 1+u^{n+1} \frac{g_{2}}{g_{2} a+g_{1}} R C \tau(C)^{-1} A \bmod u^{m+1} \\
H & =1+i_{1} \tau\left(i_{1}\right)^{-1} i_{2} B=1+u^{n} i_{1} \tau\left(i_{1}\right)^{-1} i_{2} C \tau(C)^{-1}
\end{aligned}
$$

are as in Propositions 5.1 and 5.4. As the character $\chi$ of $I_{m, \underline{w}_{m}} / I^{m}$ is inflated from a character of $U_{E} / U_{E}^{m+1}$ (again denoted by $\chi$ ), we see that

$$
\sum_{i \mapsto i_{1}} \sharp S_{g, i} \chi(i)=\sharp S_{g, i_{1}}^{\prime} \chi\left(i_{1}\right),
$$

where $i$ varies through all elements of $I_{m, \underline{w}_{m}} / I^{m}$ lying over $i_{1}$ and $\sharp S_{g, i_{1}}^{\prime}$ is the number of solutions of equations (5.5), (5.6), (5.7) in the variables $a, C, A, i_{2}$. It is enough to show that $\sharp S_{g, i_{1}}^{\prime}=$ $(q-1) q^{2 m_{\sharp}} S_{g, i_{1}}$. If $n \geqslant m+1$, then $N, H \equiv 1 \bmod u^{m+1}$ and the proof is immediate. Assume 
$n \leqslant m \leqslant 2 n$. We cancel $C$ in $(5.6)$ and insert the condition on the determinant to bring it to the form

$$
\frac{\tau\left(i_{1}\right)}{g_{2} a+g_{1}} H \equiv N \quad \bmod u^{m+1} .
$$

By replacing $N$ by $\frac{\tau\left(i_{1}\right)}{g_{2} a+g_{1}} H$ in 5.7 and canceling the invertible term $H$ we see that the equations (5.5), (5.6), (5.7) are equivalent to the three equations (5.5), (5.8) and

$$
i_{2} \equiv \frac{h(g, a) \tau\left(i_{1}\right) H D}{i_{1} C}+\frac{\tau\left(i_{1}\right) A}{g_{2} a+g_{1}}-\frac{\tau\left(i_{1}\right) H A}{i_{1}} \bmod u^{m} .
$$

Using $H \equiv 1 \bmod u^{n}$ and $D \equiv R^{-1} \tau(C) \bmod u^{n}$ equation (5.9) implies:

$$
i_{2} \equiv \frac{h(g, a) \tau\left(i_{1}\right) \tau(C)}{i_{1} R C}+\frac{\tau\left(i_{1}\right)}{g_{2} a+g_{1}} A-\frac{\tau\left(i_{1}\right) A}{i_{1}} \bmod u^{n}
$$

(the right hand side does not depend on $i_{2}$ ). We can replace $i_{2}$ occurring in the term $H$ in $(5.8)$ by the right hand side of (5.10) and hence our three original equations (5.5), (5.6), 5.7) are equivalent to (5.5),

$$
\begin{array}{r}
\tau\left(i_{1}\right)\left(1+u^{n}\left(\frac{h(g, a)}{R}+\frac{i_{1}}{g_{2} a+g_{1}} C \tau(C)^{-1} A-C \tau(C)^{-1} A\right)\right) \equiv \\
\equiv\left(g_{2} a+g_{1}\right)+u^{n+1} g_{2} R C \tau(C)^{-1} A \quad \bmod u^{m+1}
\end{array}
$$

and (5.9). By Lemma 5.8 applied to $x=i_{2}$, equation (5.9) is just an expression of $i_{2}$ in terms of $g, i_{1}, a, C, A$, hence it can be ignored and we see that $\sharp S_{g, i_{1}}^{\prime}$ is the number of solutions of 5.5 and (5.11) in the variables $a, C, A$.

Now, 5.11 implies $\tau\left(i_{1}\right) \equiv g_{2} a+g_{1} \bmod u^{n}$. Applying Lemma 5.8 to $x=\tau\left(i_{1}\right)$, we see that (5.11) is equivalent to

$$
\tau\left(i_{1}\right)+u^{n}\left(\left(g_{2} a+g_{1}\right) h(g, a) R^{-1}+\left(g_{2} \tau(a)+g_{1}\right) C \tau(C)^{-1} A-\left(g_{2} a+g_{1}\right) C \tau(C)^{-1} A\right) \equiv
$$

$$
\equiv\left(g_{2} a+g_{1}\right)+u^{n+1} g_{2} R C \tau(C)^{-1} A \bmod u^{m+1} .
$$

Inserting on the right hand side $R=u^{-1}(\tau(a)-a)$, we immediately see that 5.12 is equivalent to 5.3. Moreover, 5.5 is immediately seen to be equivalent to 5.2. As in 5.2, (5.3) neither $C$, nor $A$ occur, and as $C$ lives in $\left(k[u] / u^{m+1}\right)^{\times}$and $A$ lives in $k[u] / u^{m}$, we deduce that $\sharp S_{g, i_{1}}^{\prime}=(q-1) q^{2 m_{\sharp}} \sharp S_{g, i_{1}}$.

We now examine solutions of the equation 5.2 in $a \in L_{[1, n+m]}^{\leqslant n} \mathbb{G}_{a}(k)$ (with $a_{1} \neq 0$ ). Recall that via the embedding $\iota$ (see Section 3.1.3 we have the subgroups $U_{F} U_{\mathfrak{J}}^{n} \subseteq U_{E} U_{\mathfrak{J}}^{n} \subseteq U_{\mathfrak{J}}$.

Lemma 5.9. Let $g \in U_{\mathfrak{J}}$. Precisely one of the following cases occur:

(i) $g \in U_{F} U_{\mathfrak{J}}^{n}$. Then 5.2 has precisely $(q-1) q^{n-1}$ solutions.

(ii) $g \in U_{\mathfrak{J}} \backslash U_{F} U_{\mathfrak{J}}^{n}$ is conjugate in $U_{\mathfrak{J}}$ to an element of $U_{E} U_{\mathfrak{J}}^{n}$. In this case (5.2) has precisely $2 q^{v_{u}\left(g_{3}\right)-1}$ solutions.

(iii) $g \in U_{\mathfrak{J}} \backslash U_{F} U_{\mathfrak{J}}^{n}$ is not conjugate in $U_{\mathfrak{J}}$ to an element of $U_{E} U_{\mathfrak{J}}^{n}$. Then (5.2) has no solutions.

Proof. Assume (5.2) has a solution $a$. As $g \in U_{\mathfrak{J}}$, the integers $v_{u}\left(g_{2}\right), v_{u}\left(g_{3}\right), v_{u}\left(g_{1}-g_{4}\right)$ are even. As $a_{1} \neq 0$, we have $v_{u}(a)=1$. We deduce that $v_{u}\left(\left(g_{1}-g_{4}\right) a\right)$ is odd and $v_{u}\left(g_{2} a^{2}\right), v_{u}\left(g_{3}\right)$ are even. Thus by Lemma 5.10 we are either in the case $g \in U_{F} U_{\mathfrak{J}}^{n}$ of the lemma, where each of 
these three integers is $\geqslant n+1$ and each element of $L_{[1, n+m]}^{\leqslant n} \mathbb{G}_{a}(k)$ solves equation $(5.2)$, or we are forced to have $v_{u}\left(g_{3}\right)=v_{u}\left(g_{2}\right)+2<n+1$ and $v_{u}\left(g_{3}\right) \leqslant v_{u}\left(g_{1}-g_{4}\right)+1$ (this last is, using parity, equivalent to $\left.v_{u}\left(g_{3}\right) \leqslant v_{u}\left(g_{1}-g_{4}\right)\right)$. In the last case write $g_{2}=g_{2}^{\prime} u^{v_{u}\left(g_{2}\right)}, g_{3}=g_{3}^{\prime} u^{v_{u}\left(g_{2}\right)+2}$, $a=a^{\prime} u$ and $g_{1}-g_{4}=g_{1,4}^{\prime} u^{v_{u}\left(g_{2}\right)+2}$ with $g_{2}^{\prime}, g_{3}^{\prime}, a^{\prime} \in k \llbracket t \rrbracket^{\times}$and $g_{1,4}^{\prime} \in k \llbracket t \rrbracket$. After canceling $u^{v_{u}\left(g_{3}\right)}=u^{v_{u}\left(g_{2}\right)+2},(5.2)$ is equivalent to

$$
g_{2}^{\prime} a^{\prime, 2}+g_{1,4}^{\prime} a^{\prime} u-g_{3}^{\prime} \equiv 0 \quad \bmod u^{n+1-v_{u}\left(g_{3}\right)},
$$

where $n+1-v_{u}\left(g_{3}\right) \geqslant 1$. Reducing modulo $u$, we deduce $a_{1}^{2} \equiv \frac{g_{3}^{\prime}}{g_{2}^{\prime}} \bmod u$, which shows that $\frac{g_{3}^{\prime}}{g_{2}^{\prime}}$ mod $u$ must be a square of an element of $k^{\times}$, or, equivalently (cf. Lemma 4.15 ), that $\frac{g_{3}}{t g_{2}} \in k \llbracket t \rrbracket^{\times}$ is a square. Thus by Lemma 5.10 we deduce that we must be in case (ii) of the lemma and that in case (iii) there are no solutions. In case (ii) with notations as above, we have to determine how many solutions in $a^{\prime}=a_{1}+a_{2} u+\cdots+a_{n} u^{n-1}$ equation (5.13) has. Using induction, one now easily deduces that there are exactly two possibilities for $a_{1}$, exactly 1 possibility for each $a_{2}, \ldots, a_{n+1-v_{u}\left(g_{3}\right)}$ and exactly $q$ possibilities for each $a_{n+2-v_{u}\left(g_{3}\right)}, \ldots, a_{n}$.

Lemma 5.10. Let $g \in U_{\mathfrak{J}}$ and $n \geqslant 1$. Then

(i) $g \in U_{F} U_{\mathfrak{J}}^{n} \Leftrightarrow v_{u}\left(g_{2}\right) \geqslant n-1, v_{u}\left(g_{3}\right) \geqslant n+1, v_{u}\left(g_{1}-g_{4}\right) \geqslant n$.

(ii) $g \in U_{\mathfrak{J}} \backslash U_{F} U_{\mathfrak{J}}^{n}$ and $g$ is conjugate to an element of $U_{E} U_{\mathfrak{J}}^{n}$ if and only if $v_{u}\left(g_{3}\right)=$ $v_{u}\left(g_{2}\right)+2<n+1, v_{u}\left(g_{3}\right) \leqslant v_{u}\left(g_{1}-g_{4}\right)$ and $\frac{g_{3}}{t g_{2}} \in k \llbracket t \rrbracket^{\times}$is a square of an element in $k \llbracket u \rrbracket^{\times}$

Proof. (i): is an easy computation (use that $v_{u}\left(g_{j}\right)$ is always even). (ii): In the $\mathcal{O}_{F}$-algebra $\mathfrak{J}$ the subset $\varpi^{n} \mathfrak{J}$ form a two-sided ideal and $U_{\mathfrak{J}} / U_{\mathfrak{J}}^{n}=\left(\mathfrak{J} / \varpi^{n} \mathfrak{J}\right)^{\times}$. Assume $g \in U_{\mathfrak{J}} \backslash U_{F} U_{\mathfrak{J}}^{n}$ and $v_{u}\left(g_{3}\right)=v_{u}\left(g_{2}\right)+2<n+1, v_{u}\left(g_{3}\right) \leqslant v_{u}\left(g_{1}-g_{4}\right)$ and $\frac{g_{3}}{t g_{2}} \in k \llbracket t \rrbracket^{\times}$is a square of an element in $k \llbracket u \rrbracket^{\times}$. We replace $U_{\mathfrak{J}}$ (resp. $\left.\mathfrak{J}\right)$ by $U_{\mathfrak{J}} / U_{\mathfrak{J}}^{n}$ (resp. $\left.\mathfrak{J} / \varpi^{n} \mathfrak{J}\right)$ and $g$ by its image there. We show that $g$ is conjugate to an element of $U_{E} / U_{E}^{n}=U_{E} / U_{E} \cap U_{\mathfrak{J}}^{n}$. Replace $g$ by the difference of $g$ and the scalar matrix with entries $\frac{1}{2}\left(g_{1}+g_{4}\right)$. Thus we can assume that $g$ has trace zero and we must show that there is some $b \in \mathcal{O}_{F}$ such that $g$ is conjugate in $\mathfrak{J} / \varpi^{n} \mathfrak{J}$ to the image of $\left(\begin{array}{cc}{ }^{2} b \\ t b\end{array}\right)$. Consider $r_{y, \lambda}$ from Lemma 5.15 . Note that

$$
r_{y, \lambda}\left(\begin{array}{cc} 
& b \\
t b &
\end{array}\right) r_{y, \lambda}^{-1}=\left(\begin{array}{cc}
b \lambda t & b y^{-1}\left(1-\lambda^{2} t\right) \\
b y t & -b \lambda t
\end{array}\right)
$$

By our assumptions we can write $g_{2}=t^{\alpha} g_{2}^{\prime}, g_{3}=t^{\alpha+1} g_{3}^{\prime}, g_{1}=-g_{4}=t^{\alpha+1} g_{1}^{\prime}$ with $\alpha+1 \leqslant\left\lfloor\frac{n}{2}\right\rfloor$ and $g_{2}^{\prime}, g_{3}^{\prime} \in k \llbracket t \rrbracket^{\times}$. Thus we can conclude, if we find appropriate $y \in U_{F} / U_{F}^{\left\lfloor\frac{n+1}{2}\right\rfloor}, \lambda \in \mathcal{O}_{F} / \mathcal{O}_{F}^{\left\lfloor\frac{n}{2}\right\rfloor}$ and $b=b_{0} t^{\alpha} \in \mathcal{O}_{F}$ with $b_{0} \in U_{F}$ such that

$$
\begin{array}{rll}
b_{0} \lambda & \equiv g_{1}^{\prime} \bmod t^{\left\lfloor\frac{n+1}{2}\right\rfloor-(\alpha+1)} \\
b_{0} y & \equiv g_{3}^{\prime} \bmod t^{\left\lfloor\frac{n}{2}\right\rfloor-\alpha} \\
b_{0} y^{-1}\left(1-\lambda^{2} t\right) & \equiv g_{2}^{\prime} \bmod t^{\left\lfloor\frac{n}{2}\right\rfloor-\alpha}
\end{array}
$$

Using the first and the second equations to eliminate $b_{0}$ and $\lambda$, the only remaining equation is

$$
y^{2} \equiv g_{3}^{\prime} g_{2}^{\prime,-1}\left(1-g_{1}^{\prime \prime 2} g_{3}^{\prime,-2} y^{2} t\right) \quad \bmod t^{\left\lfloor\frac{n}{2}\right\rfloor-\alpha}
$$

This equation has a solution in $y$ by Hensel's lemma and our assumption on $\frac{g_{3}}{t g_{2}}$. The other direction in (ii) is an immediate computation.

\subsection{Traces of unipotent elements.}

In Sections 5.35.6 we assume $m=2 n-1$. 
Proof of Lemma 4.4. We use notations of Proposition 5.6. Let $g \in U_{\mathfrak{J}}^{m+1}$. Thus $v_{u}\left(g_{1}-\right.$ 1), $v_{u}\left(g_{2}\right), v_{u}\left(g_{4}-1\right) \geqslant 2 n=m+1$ and $v_{u}\left(g_{3}\right) \geqslant m+3$. This, Proposition 5.6 and Lemma 5.2 (i) show that $\sharp S_{g, i_{1}}=0$ for $i_{1} \in U_{E} / U_{E}^{m+1} \backslash\{1\}$. Lemma 5.9 implies $\sharp S_{g, 1}=(q-1) q^{n-1}$. Proposition 5.6 shows $\operatorname{tr}\left(g ; \Xi_{\chi}\right)=(q-1) q^{n-1}$.

Proof of Lemma 4.6. We use notations from Proposition 5.6. The case $g=1$ of Lemma 4.6 follows from Lemma 4.4. Write $\delta:=\left\lfloor\frac{n+1}{2}\right\rfloor-\left\lfloor\frac{n}{2}\right\rfloor$. For $0 \leqslant \alpha \leqslant\left\lfloor\frac{n+1}{2}\right\rfloor-1$ consider the subgroup

$$
A_{\alpha}:=\left\{1+u^{(n-\delta)+2 \alpha+1} y: y \text { is } \tau \text {-invariant }\right\}
$$

of $U_{E}^{(n-\delta)+2 \alpha+1} / U_{E}^{m+1}$, and let $A_{\left\lfloor\frac{n+1}{2}\right\rfloor}:=\{1\} \subseteq U_{E} / U_{E}^{m+1}$.

Lemma 5.11. Let $g \in N_{n} \backslash\{1\}$. If $g \notin N_{n}^{\left\lfloor\frac{n}{2}\right\rfloor+1}$, then $S_{g, i_{1}}=\varnothing$ for all $i_{1} \in U_{E} / U_{E}^{m+1}$. Otherwise, let $g \in N_{n}^{\left\lfloor\frac{n}{2}\right\rfloor+1+\alpha} \backslash N_{n}^{\left\lfloor\frac{n}{2}\right\rfloor+2+\alpha}$ for some $0 \leqslant \alpha \leqslant\left\lfloor\frac{n+1}{2}\right\rfloor-1$ and $i_{1} \in U_{E} / U_{E}^{m+1}$. Then

$$
\sharp S_{g, i_{1}}= \begin{cases}c(\alpha) & \text { if } i_{1} \in A_{\alpha} \backslash A_{\alpha+1} \\ 0 & \text { otherwise, }\end{cases}
$$

where $c(\alpha)$ depends only on $\alpha$, not on $i_{1}$. Moreover, $c\left(\left\lfloor\frac{n+1}{2}\right\rfloor-1\right)=q^{n-1}$.

Proof. If $g \in N_{n} \backslash N_{n}^{\left\lfloor\frac{n}{2}\right\rfloor+1}$, then $g$ is not conjugate to an element of $U_{E} U_{\mathfrak{J}}^{n}$ by Lemma 5.10, so $S_{g, i_{1}}=\varnothing$ for all $i_{1} \in U_{E} / U_{E}^{m+1}$ by Lemma 5.9 , and the first statement of the lemma follows from Proposition 5.6 (alternatively, look at equation $(5.2)$ for $g$ ). Let $g \in N_{n}^{\left\lfloor\frac{n}{2}\right\rfloor+1+\alpha} \backslash N_{n}^{\left\lfloor\frac{n}{2}\right\rfloor+2+\alpha}$ for some $0 \leqslant \alpha \leqslant\left\lfloor\frac{n+1}{2}\right\rfloor-1$ and $i_{1} \in U_{E} / U_{E}^{m+1}$. Write $g=\left(\begin{array}{cc}1 \\ t^{\left\lfloor\frac{n}{2}\right\rfloor+1+\alpha} x & 1\end{array}\right)$ with $v_{t}(x)=0$. Then equation (5.2) is trivially satisfied for each $a$ and equation (5.3) takes the form

$$
i_{1} \equiv 1+u^{(n-\delta)+2 \alpha+1} x R^{-1} \bmod u^{m+1}
$$

(one easily computes $h(g, a)=u^{2 \alpha+1-\delta} x$ ). Write $a=\sum_{i=1}^{n} a_{i} u^{i}$. Then $R=u^{-1}(\tau(a)-a)=$ $-2\left(a_{1}+a_{3} u^{2}+\ldots\right)$ and $x$ are $\tau$-invariant and we have $v_{t}(R)=v_{t}(x)=0$. Hence $S_{g, i_{1}}=$ $\varnothing$ unless $i_{1} \in A_{\alpha} \backslash A_{\alpha+1}$. On the other hand, from the explicit form of $R$, it is clear that for any $i_{1} \in A_{\alpha} \backslash A_{\alpha+1}$ the set $S_{g, i_{1}}$ of solutions $a$ of $(5.15)$ has the same cardinality. The second statement of the lemma follows. To see the last statement, put $\alpha=\left\lfloor\frac{n+1}{2}\right\rfloor-1$. Then $(n-\delta)+2 \alpha+1=2 n-1=m$ and for a fixed $i_{1} \in A_{\left\lfloor\frac{n+1}{2}\right\rfloor-1} \backslash A_{\left\lfloor\frac{n+1}{2}\right\rfloor}=U_{E}^{m} / U_{E}^{m+1} \backslash\{1\}$ equation (5.15) amounts to a condition on $R \bmod u$, or, which is the same, on $a_{1}$. It determines $a_{1}$ uniquely and $a_{2}, a_{3}, \ldots, a_{n}$ can be chosen arbitrarily. Thus (5.15) has exactly $q^{n-1}$ solutions.

Now we can finish the proof of the Lemma 4.6. Let $g \in N_{n} \backslash N_{n}^{n}$. If $g \notin N_{n}^{\left\lfloor\frac{n}{2}\right\rfloor+1}$, then Proposition 5.6 and the first statement in Lemma 5.11 immediately $\operatorname{show} \operatorname{tr}\left(g ; \Xi_{\chi}\right)=0$. Otherwise, there is some $\alpha$ with $0 \leqslant \alpha<\left\lfloor\frac{n+1}{2}\right\rfloor-1$, such that $g \in N_{n}^{\left\lfloor\frac{n}{2}\right\rfloor+1+\alpha} \backslash N_{n}^{\left\lfloor\frac{n}{2}\right\rfloor+2+\alpha}$, and we deduce from Proposition 5.6 and Lemma 5.11

$$
\operatorname{tr}\left(g ; \Xi_{\chi}\right)=\sum_{i_{1} \in A_{\alpha} \backslash A_{\alpha+1}} c(\alpha) \chi\left(i_{1}\right)=c(\alpha) \sum_{i_{1} \in A_{\alpha}} \chi\left(i_{1}\right)-c(\alpha) \sum_{i_{1} \in A_{\alpha+1}} \chi\left(i_{1}\right)=0,
$$

as $A_{\alpha}, A_{\alpha+1}$ both are subgroups containing $U_{E}^{m} / U_{E}^{m+1}$ and $\chi$ is a non-trivial character on $U_{E}^{m} / U_{E}^{m+1}$. Now assume $g \in N_{n}^{n} \backslash\{1\}$. This corresponds to $\alpha=\left\lfloor\frac{n+1}{2}\right\rfloor-1$ and $A_{\left\lfloor\frac{n+1}{2}\right\rfloor-1}=$ $U_{E}^{m} / U_{E}^{m+1}$. By Proposition 5.6 and Lemma $\frac{5.11}{37}$ we compute 


$$
\operatorname{tr}\left(g ; \Xi_{\chi}\right)=\sum_{i_{1} \in U_{E}^{m} / U_{E}^{m+1} \backslash\{1\}} q^{n-1} \chi\left(i_{1}\right)=-q^{n-1},
$$

as $\chi$ is non-trivial on $U_{E}^{m} / U_{E}^{m+1}$. This finishes the proof of Lemma 4.6 .

\subsection{Traces of some non-split elements.}

Proposition 5.12. Let $0 \leqslant \alpha \leqslant n-1$. Let $g=\iota\left(1+u^{2 \alpha+1} h\right)$ for some $h \in U_{F}$. Then

$$
\operatorname{tr}\left(g ; \Xi_{\chi}\right)=q^{\alpha}\left(\chi(g)+\chi^{\tau}(g)\right)+2 q^{\alpha} \cdot \sum_{\substack{i_{1}=1+u^{2 \alpha+1} h s \\ s \in Q_{\alpha}}} \chi\left(i_{1}\right),
$$

with $Q_{\alpha}$ as in Section 4.4.7.

Proof. This follows immediately from Proposition 5.6 and Lemma 5.13 .

Lemma 5.13. Let $\alpha, g, h$ be as in Proposition 5.12. Then $S_{g, i_{1}}=\varnothing$, unless $i_{1}=1+u^{2 \alpha+1} h s$ for some $s \in R_{\alpha}$. Assume this holds. Then

$$
S_{g, i_{1}}= \begin{cases}2 q^{\alpha} & \text { if } s \in Q_{\alpha} \\ q^{\alpha} & \text { if } s= \pm 1 \\ 0 & \text { otherwise. }\end{cases}
$$

Proof. Assume first $\alpha \geqslant\left\lfloor\frac{n}{2}\right\rfloor$, or equivalently $2 \alpha+1 \geqslant n$. In this case $\sharp S_{g, i_{1}}$ is equal to the number of solutions of (5.3) in the variable $a$. Using Lemma 5.2 (ii), we see that $S_{g, i_{1}}=\varnothing$, unless $i_{1}=1+u^{2 \alpha+1} h s$ for some $s \in R_{\alpha}$. Assume this holds. As $h$ is $\tau$-invariant, it follows that the condition $\operatorname{det}(g) \equiv i_{1} \tau\left(i_{1}\right) \bmod u^{m+1}$ (necessary for the non-emptiness of $S_{g, i_{1}}$ ) is equivalent to $s \in R_{\alpha}^{\langle\tau\rangle}$. Thus we can assume that $i_{1}=1+u^{2 \alpha+1} h s$ with $s \in R_{\alpha}^{\langle\tau\rangle}$. Then, using Lemma 5.2(ii), (5.3) is seen to be equivalent to

$$
s \equiv\left(1-a^{\prime, 2}\right)\left(1-u^{2 \alpha+1} h a^{\prime}\right) R^{-1}-a^{\prime} \bmod u^{m-2 \alpha},
$$

where we write $a=u a^{\prime}$. By assumption $2 \alpha+1 \geqslant m-2 \alpha$, and moreover, $R=u^{-1}(\tau(a)-a)=$ $-\left(\tau\left(a^{\prime}\right)+a^{\prime}\right)$. Hence 5.3$)$ is equivalent to

$$
s \equiv-\frac{1+a^{\prime} \tau\left(a^{\prime}\right)}{a^{\prime}+\tau\left(a^{\prime}\right)} \quad \bmod u^{m-2 \alpha} .
$$

Assume this equation has a solution in $a^{\prime}$. Then we deduce

$$
s \tau(s)-1=s^{2}-1=\left(\frac{1+a^{\prime} \tau\left(a^{\prime}\right)}{a^{\prime}+\tau\left(a^{\prime}\right)}\right)^{2}-1=\mathrm{N}_{\tau, \alpha}\left(\frac{1-a^{\prime, 2}}{a^{\prime}+\tau\left(a^{\prime}\right)}\right)
$$

in $R_{\alpha}$. This shows that if $S_{g, i_{1}} \neq \varnothing$, then $s \in Q_{\alpha} \cup\{ \pm 1\}$. Conversely, assume that $s \in Q_{\alpha} \cup\{ \pm 1\}$. Write $a^{\prime}=a_{1}+a_{2} u+\cdots+a_{n} u^{n-1}$. We differ between three cases.

Case 1. $s \not \equiv \pm 1 \bmod u$. Let $s_{0}:=s \bmod u$. By Lemma 4.17, $s_{0}^{2}-1$ is a square in $k^{\times}$. We can rearrange the equation (5.17) and bring it to the form

$$
\begin{aligned}
\left(1+a_{1}^{2}\right) & +\left(2 a_{1} a_{3}-a_{2}^{2}\right) u^{2}+\cdots+\left(2 \sum_{j=0}^{i-1}(-1)^{j} a_{j+1} a_{2 i-j+1}+(-1)^{i} a_{i+1}^{2}\right) u^{2 i}+\ldots \\
& \ldots+\left(2 a_{1} a_{2\left\lfloor\frac{n+1}{2}\right\rfloor-1}+\ldots\right) u^{2\left\lfloor\frac{n+1}{2}\right\rfloor-2} \equiv-2 s\left(a_{1}+a_{3} u^{2}+\cdots+a_{2\left\lfloor\frac{n+1}{2}\right\rfloor-1} u^{2\left\lfloor\frac{n+1}{2}\right\rfloor-2}\right) \quad \bmod u^{m-2 \alpha} .
\end{aligned}
$$

Taking this equation modulo $u$, we obtain the equation $a_{1}^{2}+2 s_{0} a_{1}+1=0 \mathrm{in} k$. It has precisely two different solutions in $a_{1}$ as $s_{0}^{2}-1$ is a square in $k^{\times}$. Note that both solutions satisfy $a_{1} \neq-s_{0}$ due 
to $s_{0} \neq \pm 1$. Taking the above equation iteratively modulo $u^{3}, \ldots, u^{m-2 \alpha}$ and using $a_{1} \neq-s_{0}$, we see that there are exactly $q$ possibilities to choose any of the pairs $\left(a_{2}, a_{3}\right), \ldots,\left(a_{m-2 \alpha-1}, a_{m-2 \alpha}\right)$ and we obtain $q$ possibilities for each of the remaining variables $a_{m-2 \alpha}, \ldots, a_{n}$ (note that $m-$ $\left.2 \alpha \leqslant 2\left\lfloor\frac{n+1}{2}\right\rfloor-1\right)$. Altogether we obtain $2 q^{n-\alpha-1} q^{n-(m-2 \alpha)}=2 q^{\alpha}$ solutions.

Case 2. $v_{u}(s+1)=2 j$ or $v_{u}(s-1)=2 j$ with $0<2 j<m-2 \alpha$ (note that the $v_{u}(s \pm 1$ ) has to be even, as $s$ is $\tau$-invariant). We assume $v_{u}(s-1)=2 j$ (the other case is similar). Then we can write $s=1+u^{2 j} s^{\prime}$ for some $\tau$-invariant unit $s^{\prime}$. Then (5.17) is equivalent to

$$
\left(1+a^{\prime}\right)\left(1+\tau\left(a^{\prime}\right)\right) \equiv-u^{2 j} s^{\prime}\left(a^{\prime}+\tau\left(a^{\prime}\right)\right) \quad \bmod u^{m-2 \alpha},
$$

and we deduce that a solution $a^{\prime}$ must satisfy $v_{u}\left(1+a^{\prime}\right)=j$ (as $s^{\prime}, a^{\prime}+\tau\left(a^{\prime}\right)$ are necessarily units and $\left.v_{u}\left(1+a^{\prime}\right)=v_{u}\left(1+\tau\left(a^{\prime}\right)\right)\right)$. Set $a^{\prime}=-1+u^{j} b$ with some $b=\sum_{i=0}^{n-j-1} b_{i} u^{i} \in\left(k[u] / u^{n-j}\right)^{\times}$. The number of solutions of (5.17) in $a^{\prime}$ is equal to the number of solutions of

$$
(-1)^{j} b \tau(b) \equiv s^{\prime}\left(2-u^{j}\left(b+(-1)^{j} \tau(b)\right)\right) \quad \bmod u^{m-2 \alpha-2 j}
$$

in the variable $b \in\left(k[u] / u^{n-j}\right)^{\times}$. Taking this equation modulo $u$ we get the equation $(-1)^{j} b_{0}^{2} \equiv$ $2 s^{\prime} \bmod u$. As $s=1+u^{2 j} s^{\prime} \in Q_{\alpha}$, Lemma 4.17 shows that $(-1)^{j} 2 s^{\prime} \bmod u$ is a square in $k^{\times}$, and thus this equation has exactly two solutions in $b_{0}$. Similarly as in case 1 above, taking (5.18) iteratively modulo $u^{3}, u^{5}, \ldots, u^{m-2 \alpha-2 j}$, we get per step exactly one condition which determines $b_{2}, b_{4}, \ldots, b_{m-2 \alpha-2 j-1}$ uniquely (note: the set of these conditions also can be empty). For each $b_{i}$ with $i \notin\{0,2,4, \ldots, m-2 \alpha-2 j-1\}$ there are $q$ possible choices. Thus the number of solutions of (5.18) in $b$ is equal to $2 q^{(n-j-1)-(n-\alpha-j-1)}=2 q^{\alpha}$.

Case 3. $s= \pm 1$. Assume $s=1$ (the other case is similar). Then (5.17) is equivalent to

$$
\left(1+a^{\prime}\right)\left(1+\tau\left(a^{\prime}\right)\right) \equiv 0 \quad \bmod u^{m-2 \alpha},
$$

which in turn is equivalent to $v_{u}\left(1+a^{\prime}\right) \geqslant \frac{m-2 \alpha+1}{2}=n-\alpha$. We easily deduce that the number of solutions of this equation in $a^{\prime}$ is equal to $q^{\alpha}$. This finishes the case $\alpha \geqslant\left\lfloor\frac{n}{2}\right\rfloor$.

Assume now $0 \leqslant \alpha<\left\lfloor\frac{n}{2}\right\rfloor$. Then $2 \alpha+1<n$. The quantity $\sharp S_{g, i_{1}}$ is equal to the number of solutions of $(5.2)$ and (5.3) in $a$. We again write $a=u a^{\prime}$. Equation 5.2 is immediately seen to be equivalent to $a^{\prime} \equiv \pm 1 \bmod u^{n-2 \alpha-1}$ and we write $a^{\prime}= \pm 1+u^{n-2 \alpha-1} b$ for $b \in k[u] / u^{2 \alpha+1}$. We deduce

$$
R \equiv-\left(a^{\prime}+\tau\left(a^{\prime}\right)\right) \equiv \mp 2-u^{n-2 \alpha-1}\left(b+(-1)^{n+1} \tau(b)\right) \quad \bmod u^{m+1} .
$$

Let us denote the 'automorphic factor' $g_{2} a+g_{1}$ by

$$
f:=g_{2} a+g_{1}=1 \pm u^{2 \alpha+1} h+u^{n} h b .
$$

By Lemma 5.2 (iii), the quantity $\sharp S_{g, i_{1}}$ is equal to the number of solutions in the variable $b \in$ $k[u] / u^{2 \alpha+1}$ of the equation

or equivalently,

$$
\tau\left(i_{1}\right)\left(1+u^{n} R^{-1} \frac{h\left(\mp 2 b-u^{n-2 \alpha-1} b^{2}\right)}{f}\right) \equiv f \bmod u^{m+1},
$$

$$
\tau\left(i_{1}\right) \equiv f-u^{n} R^{-1} h\left(\mp 2 b-u^{n-2 \alpha-1} b^{2}\right) \quad \bmod u^{m+1} .
$$

Taking this equation modulo $u^{m-2 \alpha}=u^{2 n-2 \alpha-1}$ and using (5.19) and (5.20), we deduce that $S_{g, i_{1}}=\varnothing$, unless $i_{1} \equiv 1 \mp u^{2 \alpha+1} h \bmod u^{m-2 \alpha}$, or with other words, $i_{1}=1+u^{2 \alpha+1} h s$ with $s \in R_{\alpha}$ satisfying $s \equiv \mp 1 \bmod u^{m-4 \alpha-1}$. Assume that this holds. An easy computation shows 
now that $\operatorname{det}(g) \equiv i_{1} \tau\left(i_{1}\right) \bmod u^{m+1}$ is equivalent to $s \in R_{\alpha}^{\langle\tau\rangle, \prime}$, so we also can assume this (otherwise, $S_{g, i_{1}}=\varnothing$ ). Let us write $s=\mp 1+u^{m-4 \alpha-1} \cdot\left(u^{2 j} s_{0}\right)$, with $s_{0} \in\left(k[u] / u^{2 \alpha-2 j+1}\right)^{\times} \tau$ invariant with $0 \leqslant j \leqslant \alpha+1(j=\alpha+1$ corresponds to $s= \pm 1)$. Straightforward rearrangements of terms show that (5.21) is equivalent to

$$
\left(\mp 2-\left(b+(-1)^{n+1} \tau(b)\right) u^{n-2 \alpha-1}\right) u^{2 j} s_{0} \equiv(-1)^{n+1} b \tau(b) \quad \bmod u^{2 \alpha+1} .
$$

If $j=\alpha+1$, then $s= \pm 1$ and $(5.22)$ is equivalent to $b \tau(b) \equiv 0 \bmod u^{2 \alpha+1}$. This is equivalent to $b \equiv 0 \bmod u^{\alpha+1}$, and hence $(5.22)$ has precisely $q^{\alpha}$ solutions in $b$. Assume $j \leqslant \alpha$. A potential solution $b$ of $(5.22)$ must satisfy $b \equiv 0 \bmod u^{j}$, hence we can write $b=u^{j} b^{\prime}$ for a $b^{\prime} \in k[u] / u^{2 \alpha+1-j}$ and rewrite $(5.22)$ as

$$
\left(\mp 2-\left(b^{\prime}+(-1)^{n+j+1} \tau\left(b^{\prime}\right)\right) u^{n-2 \alpha-1+j}\right) s_{0} \equiv(-1)^{n+j+1} b^{\prime} \tau\left(b^{\prime}\right) \quad \bmod u^{2 \alpha+1-2 j} .
$$

Assume first $S_{g, i_{1}} \neq \varnothing$, i.e., (5.23) has at least one solution. Taking (5.23) modulo $u$, we deduce that $\pm(-1)^{n+j} 2 s_{0} \bmod u$ is a square in $k^{\times}$, which is by Lemma 4.17 equivalent to $s \in Q_{\alpha}$. Thus $S_{g, i_{1}} \neq \varnothing$ implies $s \in Q_{\alpha}$. Conversely, if $s \in Q_{\alpha}$, we can deduce that $\sharp S_{g, i_{1}}=2 q^{\alpha}$ in the same way as in the case $\alpha \geqslant\left\lfloor\frac{n}{2}\right\rfloor$.

We are convinced that there must be a more elegant proof of Lemma 5.13, but we still can not find it.

\subsection{Traces of elements in $E^{\times}$with $u$-valuation 1 .}

Proof of Proposition 4.22. Put $y_{1}:=e_{0}(u,-u)$. Consider the automorphism $\tilde{\beta}_{g}: \tilde{Y}_{\dot{w}}^{m} \rightarrow \tilde{Y}_{\dot{w}}^{m}$ given by $\tilde{\beta}_{g}(\dot{x} I)=g \dot{x} y_{1}^{-1} I^{m}$. Then $\tilde{\beta}_{g}$ induces an automorphism of $\mathrm{H}_{c}^{0}\left(\tilde{Y}_{\dot{w}}^{m}\right)$ and hence also an automorphism $\tilde{\beta}_{g}^{*}: V_{\chi} \rightarrow V_{\chi}$ of its $\chi$-isotypic quotient. As $y_{1}$ acts in $V_{\chi}$ as the scalar multiplication ${ }^{2}$ with $\chi(u)$, we have $\operatorname{tr}\left(g ; \Xi_{\chi}\right)=\chi(u) \operatorname{tr}\left(\tilde{\beta}_{g}^{*} ; V_{\chi}\right)$. We determine $\operatorname{tr}\left(\tilde{\beta}_{g}^{*} ; V_{\chi}\right)$. As $v_{u}(g)=1$, Lemma 3.12 shows that $g Y_{\dot{w}}^{m}=Y_{\dot{w}}^{m} y_{1}$. With other words, $\tilde{\beta}_{g}$ restricts to an automorphism $\beta_{g}$ of $Y_{\dot{w}}^{m}$. Further, $\beta_{g}$ induces an automorphism $\beta_{g}^{*}$ of $\mathrm{H}_{c}^{0}\left(Y_{\dot{w}}^{m}\right)\left[\left.\chi\right|_{U_{E}}\right]$. Moreover, the isomorphism from Lemma 5.7 induces a commutative diagram

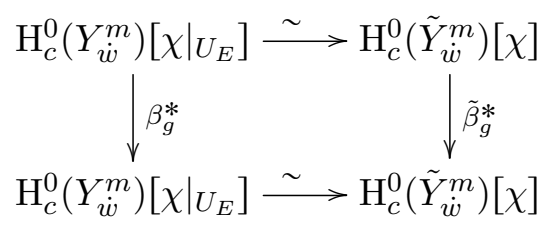

from which we deduce $\operatorname{tr}\left(\tilde{\beta}_{g}^{*} ; V_{\chi}\right)=\operatorname{tr}\left(\beta_{g}^{*} ; \mathrm{H}_{c}^{0}\left(Y_{\dot{w}}^{m}\right)\left[\left.\chi\right|_{U_{E}}\right]\right)$. Lemma 5.14 finishes the proof.

Lemma 5.14. Let $g \in E^{\times}$with $v_{u}(g)=1$. Let $\beta_{g}$ be the automorphism of $Y_{\dot{w}}^{m}$ defined by $\beta_{g}\left(\dot{x} I^{m}\right)=g \dot{x} y_{1}^{-1} I^{m}$. Write $g=g_{0} u$. Then

$$
\operatorname{tr}\left(\beta_{g}^{*} ; \mathrm{H}_{c}^{0}\left(Y_{\dot{w}}^{m}\right)\left[\left.\chi\right|_{U_{E}}\right]\right)=\chi\left(g_{0}\right)+\chi^{\tau}\left(-g_{0}\right) .
$$

Proof. Multiplying with some central element in $U_{F} \subseteq U_{E}$ (those act as scalars in $V_{\chi}$ ) we can assume that $g_{0}=1+u^{2 \alpha+1} h$ for some $h \in U_{F}$ (and $0 \leqslant \alpha<n$ ). We proceed analogously as in the proof of Proposition 5.6. Let $i \in I_{m, \underline{w}_{m}} / I^{m}$. A point $\dot{x} I \in Y_{\dot{w}}^{m}$ can lie in the set $S_{\beta_{g}, i}=\left\{\dot{x} I \in Y_{\dot{w}}^{m}: \beta_{g}\left(\dot{x} I^{m}\right)=\dot{x} i I^{m}\right\}$ from Lemma 5.5 only if $\beta_{g}$ fixes its $a$-coordinate. By Lemma 3.12, $\beta_{u}$ acts on the coordinate $a$ by $a=u a^{\prime} \mapsto u a^{\prime,-1}$. From this and Proposition 5.1

2 A subtlety: we suppressed our choice of an identification of $E^{\times}$with the diagonal quotient of $\tilde{I}_{m, w}$, for which we silently have chosen that $u$ corresponds to $y_{1}$. This choice determines on the one hand that $y_{1}$ acts in $V_{\chi}$ by $\chi(u)$, and on the other hand, that we have to evaluate the trace formula using the identifications $\varpi \leftrightarrow u \leftrightarrow y_{1}=$ $e_{0}(u,-u)$. 
one easily deduces that $\beta_{g}^{*}(a)=a$ is equivalent to $a= \pm u$ (for any $g_{0}$ ) and that $h(g, \pm u)=0$. Apply Propositions 5.1, 5.3, 5.4 to determine the actions of $\beta_{g}$ and $i$ on $Y_{\dot{w}}^{m}$. Exactly as in the proof of Proposition 5.6 we see that

$$
\operatorname{tr}\left(\beta_{g}^{*} ; \mathrm{H}_{c}^{0}\left(Y_{\dot{w}}^{m}\right)\left[\left.\chi\right|_{U_{E}}\right]\right)=\frac{1}{(q-1) q^{2 m}} \sum_{i_{1} \in U_{E} / U_{E}^{m+1}} \sharp S_{\beta_{g}, i_{1}} \chi\left(i_{1}\right),
$$

where $S_{\beta_{g}, i_{1}}$ is the set of all solutions of the equations

$$
\begin{aligned}
\left(1 \mp u^{2 \alpha+1} h\right)(\mp C) M^{-1} N^{-1} & \equiv C i_{1} H^{-1} \bmod u^{m+1} \\
-A M N & \equiv i_{1}^{-1} \tau\left(i_{1}\right) H^{2} A+i_{2} H \quad \bmod u^{m}
\end{aligned}
$$

in the variables $C \in\left(k[u] / u^{m+1}\right)^{\times}, A, i_{2} \in k[u] / u^{m}$ (the sign \pm corresponds to the two possibilities $a= \pm u$ ), where

$$
\begin{aligned}
M & =1-2 u^{n} C \tau(C)^{-1} A \text { as in Proposition 5.3, and } \\
N & =1+u^{n+1} \frac{g_{2}}{g_{2}( \pm 1)+g_{1}}\left(\mp C M^{-1}\right)(\mp D M)^{-1}(-A M)=1 \pm 2 u^{n} \frac{u^{2 \alpha+1} h}{1 \pm u^{2 \alpha+1} h} C \tau(C)^{-1} A \\
H & =1+i_{1} \tau\left(i_{1}\right)^{-1} i_{2} B=1+u^{n} i_{1} \tau\left(i_{1}\right)^{-1} i_{2} C \tau(C)^{-1} .
\end{aligned}
$$

Canceling $C$ in (5.24) we see that it is equivalent to

$$
M N i_{1} \equiv \mp\left(1 \mp u^{2 \alpha+1} h\right) H \quad \bmod u^{m+1} .
$$

Taking (5.26) modulo $u^{n}$, we see that $S_{\beta_{g}, i}=\varnothing$, unless $i_{1} \equiv \mp\left(1 \mp u^{2 \alpha+1} h\right) \bmod u^{n}$. Assume the last holds. Taking equation (5.25) modulo $u^{n}$ and inserting $M N$ from (5.26) and $i_{1} \equiv$ $\mp\left(1 \mp u^{2 \alpha+1} h\right) \bmod u^{n}$ we deduce

$$
i_{2} \equiv-A \frac{2}{1 \mp u^{2 \alpha+1} h} \quad \bmod u^{n} .
$$

This allows to compute

$$
H=1-2 u^{n} \frac{1}{1 \pm u^{2 \alpha+1} h} C \tau(C)^{-1} A .
$$

As in the proof of Proposition 5.6 we eliminate $i_{2}$ and ignore equation 5.25 . Thus $\sharp S_{\beta_{g}, i_{1}}$ is equal to the number of solutions of (5.26) in $C$ and $A$. Finally, we compute $M N=H$ (this uses $i_{1} \equiv \mp\left(1 \mp u^{2 \alpha+1} h\right) \bmod u^{n}$ and (5.27) $)$ and canceling these terms in (5.26) shows that $S_{\beta_{g}, i_{1}}=\varnothing$, unless $i_{1}=\mp\left(1 \mp u^{2 \alpha+1} h\right)$, in which case $\sharp S_{\beta_{g}, i_{1}}=(q-1) q^{2 m}$, finishing the proof.

\subsection{Traces on the induced side.}

Proof of Lemma 4.28. In $\Xi_{\chi}$ and $\Theta_{\chi}$ the central characters are $\left.\chi\right|_{F^{\times}}$and $U_{E}^{m+1}$ acts trivial. Thus it is enough to show

$$
\begin{aligned}
& \operatorname{tr}\left(g ; \Xi_{\chi}\right)=\operatorname{tr}\left(g ; \Theta_{\chi}\right) \quad \forall g=\iota\left(1+u^{2 \alpha+1} h\right) \text { with } h \in U_{F} \text { and } 0 \leqslant \alpha \leqslant n-1 \\
& \operatorname{tr}\left(g ; \Xi_{\chi}\right)=\operatorname{tr}\left(g ; \Theta_{\chi}\right) \quad \forall g \in \varpi U_{E} .
\end{aligned}
$$

Lemma 5.15. Let $n \geqslant 1$. For $y \in U_{F} / U_{F}^{\left\lfloor\frac{n+1}{2}\right\rfloor}, \lambda \in \mathcal{O}_{F} / \mathcal{O}_{F}^{\left\lfloor\frac{n}{2}\right\rfloor}$ the matrices 


$$
r_{y, \lambda}:=\left(\begin{array}{cc}
1 & \\
& y
\end{array}\right)\left(\begin{array}{cc}
1 & \lambda \\
& 1
\end{array}\right) \in U_{\mathfrak{J}} / U_{\mathfrak{J}}^{n}
$$

for a system of representatives for the left (and right) cosets of $U_{E} U_{\mathfrak{J}}^{n}$ in $U_{\mathfrak{J}}$ and hence also of $J_{\beta}=E^{\times} U_{\mathfrak{J}}^{n}$ in $E^{\times} U_{\mathfrak{J}}$.

Proof. We have $U_{\mathfrak{J}} / U_{E} U_{\mathfrak{J}}^{n} \cong E^{\times} U_{\mathfrak{J}} / E^{\times} U_{\mathfrak{J}}^{n}$. The rest is an immediate computation.

We use notations from Section 4.1.6 and compute the traces $\operatorname{tr}\left(g ; \Theta_{\chi}\right)$. Let $g=\iota\left(1+u^{2 \alpha+1} h\right)$ be as in (5.28). Applying the Mackey formula to $\Theta_{\chi}=\operatorname{Ind}_{J_{\beta}}^{E^{\times} U_{\mathfrak{J}}} \Lambda$ we see

$$
\operatorname{tr}\left(g ; \Theta_{\chi}\right)=\sum_{y, \lambda} \Lambda\left(r_{y, \lambda} g r_{y, \lambda}^{-1}\right),
$$

where the sum is taken over all representatives $r_{y, \lambda}$ of $E^{\times} U_{\mathfrak{J}} / J_{\beta}$ (from Lemma 5.15), such that $r_{y, \lambda} g r_{y, \lambda}^{-1} \in J_{\beta}=E^{\times} U_{\mathfrak{J}}^{n}$. We compute:

$$
r_{y, \lambda} g r_{y, \lambda}^{-1}=\left(\begin{array}{cc}
1+\lambda h t^{\alpha+1} & y^{-1} h\left(1-\lambda^{2} t\right) t^{\alpha} \\
y h t^{\alpha+1} & 1-\lambda h t^{\alpha+1}
\end{array}\right) .
$$

Write $\beta=(b+u c) u^{-m}$ with some $b, c \in \mathcal{O}_{F}$. Assume first $\alpha \geqslant\left\lfloor\frac{n}{2}\right\rfloor$. Then $r_{y, \lambda} g r_{y, \lambda}^{-1} \in U_{\mathfrak{J}}^{n} \subseteq U_{E} U_{\mathfrak{J}}^{n}$ for all $r_{y, \lambda}$ and we compute:

$$
\operatorname{tr}\left(g ; \Theta_{\chi}\right)=\sum_{y, \lambda} \psi_{\mathfrak{M}, \beta}\left(r_{y, \lambda} g r_{y, \lambda}^{-1}\right)=\sum_{y, \lambda} \psi\left(t^{\alpha+1-n} b h\left(y+y^{-1}\left(1-\lambda^{2} t\right)\right)\right) .
$$

Taking some lifts of $y, \lambda$ to $E$ and setting $n:=\frac{1}{2} h\left(y+y^{-1}\left(1-\lambda^{2} t\right)\right) u^{2 \alpha+1} \in E$, we see that $\beta n+\tau(\beta n)=t^{\alpha+1-n} b h\left(y+y^{-1}\left(1-\lambda^{2} t\right)\right)$, i.e., using (4.3), we deduce

$$
\operatorname{tr}\left(g ; \Theta_{\chi}\right)=\sum_{y, \lambda} \psi_{E}(\beta n)=\sum_{y, \lambda} \chi\left(1+\frac{1}{2} h\left(y+y^{-1}\left(1-\lambda^{2} t\right)\right) u^{2 \alpha+1}\right) .
$$

This does not depend on the choice of the lifts $y, \lambda$ to $E$, as $\chi$ is of level $m$. Interpreting $1+\frac{1}{2} h\left(y+y^{-1}\left(1-\lambda^{2} t\right)\right) u^{2 \alpha+1}$ as an element of $U_{E} / U_{E}^{m+1}$, we have to show that the summand $\chi\left(i_{1}\right)$ for $i_{1} \in U_{E} / U_{E}^{m+1}$ occurs in this sum if and only if and with the same multiplicity as it occurs in the sum (5.16). Therefore, writing $i_{1}=1+u^{2 \alpha+1} h s$, it is enough to show that for a fixed $s \in R_{\alpha}^{\langle\tau\rangle}$ the equation

$$
\frac{1}{2}\left(y+y^{-1}\left(1-\lambda^{2} t\right)\right) \equiv s \quad \bmod t^{n-\alpha}
$$

in the variables $y \in U_{F} / U_{F}^{\left\lfloor\frac{n+1}{2}\right\rfloor}, \lambda \in \mathcal{O}_{F} / \mathcal{O}_{F}^{\left\lfloor\frac{n}{2}\right\rfloor}$ is equivalent to the equation (5.17) in the variable $a^{\prime}=a_{1}+a_{2} u+\cdots+a_{n} u^{n-1} \in\left(k[u] / u^{n}\right)^{\times}$. Indeed, write $a^{\prime}=-b^{\prime}+c^{\prime} u$ with $b^{\prime}=-\frac{1}{2}\left(\tau\left(a^{\prime}\right)+a^{\prime}\right)=$ $\sum_{j=0}^{\left\lfloor\frac{n+1}{2}\right\rfloor-1} b_{j} t^{j}$ and $c^{\prime} u=\frac{1}{2}\left(\tau\left(a^{\prime}\right)-a^{\prime}\right)=u \sum_{j=0}^{\left\lfloor\frac{n}{2}\right\rfloor-1} c_{j} t^{j}$ with $b^{\prime}, c^{\prime} \tau$-invariant. Then 5.17) can be rewritten as

$$
s \equiv \frac{1+b^{\prime, 2}-c^{\prime, 2} t}{2 b^{\prime}} \bmod t^{n-\alpha}
$$

which is evidently equivalent to (5.32) (replace $b^{\prime}$ by $y$ and $c^{\prime}$ by $\lambda$ ). The case $\alpha<\left\lfloor\frac{n}{2}\right\rfloor$ can be done similarly. This shows (5.28).

To show 5.29), we let $g=\iota\left(u(1+h u)\right.$ ) for some $h \in \mathcal{O}_{F}$ (restriction to this case is possible after multiplication with a central element). We compute 


$$
\varpi^{-1} r_{y, \lambda} g r_{y, \lambda}^{-1}=\left(\begin{array}{cc}
y & h-\lambda \\
(h+\lambda) t & y^{-1}\left(1-\lambda^{2} t\right)
\end{array}\right) .
$$

Notice that $r_{y, \lambda} g r_{y, \lambda}^{-1} \in J_{\beta}=E^{\times} U_{\mathfrak{J}}^{n}$ if and only if $\varpi^{-1} r_{y, \lambda} g r_{y, \lambda}^{-1} \in E^{\times} U_{\mathfrak{J}}^{n} \cap U_{\mathfrak{J}}=U_{E} U_{\mathfrak{J}}^{n}$. By (5.33), this is the case if and only if $\lambda=0, y= \pm 1$. Thus $\operatorname{tr}\left(g ; \Theta_{\chi}\right)=\chi(g)+\chi^{\tau}(g)$. Together with Proposition 4.22 it shows $(5.29)$ and thus the lemma.

\subsection{Computation of traces in the small level case.}

In this section we assume $n \geqslant m+1$.

Proof of Lemma 4.30. Let $g \in U_{\mathfrak{J}}$. We apply Proposition 5.6. Observe first that equation (5.3) reduces to

$$
\tau\left(i_{1}\right) \equiv g_{2} a+g_{1} \quad \bmod u^{m+1} .
$$

(i): Then we are exactly in the case (i) of Lemma 5.9. As $v_{u}(a)=1$ and $v_{u}\left(g_{2}\right) \geqslant 2\left\lfloor\frac{n}{2}\right\rfloor$, we see that $g_{2} a+g_{1} \equiv g_{1} \bmod u^{n}$ and hence also $g_{2} a+g_{1} \equiv g_{1} \bmod u^{m+1}$. Let $i_{1} \in\left(k[u] / u^{m+1}\right)^{\times}$. Then (5.34) simply says that either $i_{1}$ is $g_{1} \bmod u^{m+1}$ or $S_{g, i_{1}}=\varnothing$. By Proposition 5.6 we deduce

$$
\operatorname{tr}\left(g ; \mathrm{H}_{c}^{0}\left(\tilde{Y}_{\dot{w}}\right)[\chi]\right)=(q-1) q^{n-1} \chi\left(g_{1}\right)=(q-1) q^{n-1} \tilde{\chi}(g),
$$

showing the first statement of (i). The last statement of (i) follows immediately from the first, as $\tilde{\chi}$ is trivial on $U_{\mathfrak{J}}^{n}$.

(ii): Conjugating $g$ into $U_{E} U_{\mathfrak{J}}^{n}$ and multiplying with an element of $U_{F} U_{\mathfrak{J}}^{n}$ (these elements act by part (i) as scalars), we can without loss of generality assume that $g=\iota\left(1+u^{2 \alpha+1} h\right)$ with some $h \in U_{F}$ and with $2 \alpha+2=v_{u}\left(g_{3}\right) \leqslant n$. Let $i_{1} \in U_{E} / U_{E}^{m+1}$. We determine $\sharp S_{g, i_{1}}$. First of all 5.2 is equivalent to

$$
\left(t^{\alpha} h\right) a^{2}-t^{\alpha+1} h \equiv 0 \quad \bmod u^{n+1} .
$$

Write $a=a^{\prime} u$ with $a^{\prime}$ invertible. This equation is equivalent to

$$
a^{\prime} \equiv \pm 1 \quad \bmod u^{n+1-(2 \alpha+2)} .
$$

Equation 5.34 takes the form

$$
\tau\left(i_{1}\right)=1+u^{2 \alpha+1} h a^{\prime} \bmod u^{m+1} .
$$

Thus (5.35) and $n \geqslant m+1$ shows that either $S_{g, i_{1}}=\varnothing$, or $i_{1}=1 \pm u^{2 \alpha+1} h$. Moreover, for each of this two choices of $i_{1}$, there are exactly $q^{v_{u}\left(g_{3}\right)-1}=q^{2 \alpha+1}$ possible $a$ 's satisfying equations (5.35) and (5.34) (cf. Lemma 5.9(ii)). We obtain

$$
\operatorname{tr}\left(g ; \mathrm{H}_{c}^{0}\left(\tilde{Y}_{\dot{w}}\right)[\chi]\right)=q^{v_{u}\left(g_{3}\right)-1} \cdot\left(\tilde{\chi}(g)+\tilde{\chi}^{\tau}(g)\right) .
$$

(iii): By Lemma 5.9(iii) it is clear that $S_{g, i_{1}}=\varnothing$ for all $i_{1}$ in this case.

Let now $g=g_{0} \varpi \in \varpi U_{E}$. As in the proof of Proposition 4.22 we have the automorphism $\tilde{\beta}_{g}$ of $\tilde{Y}_{\dot{w}}^{m}$ defined by $\tilde{\beta}_{g}\left(\dot{x} I^{m}\right)=g \dot{x} y_{1}^{-1} I^{m}$, where $y_{1}=e_{0}(u,-u)$ and its restriction $\beta_{g}$ to $Y_{\dot{w}}^{m}$. Again, we have

$$
\operatorname{tr}\left(g ; \mathrm{H}_{c}^{0}\left(\tilde{Y}_{\dot{w}}^{m}\right)[\chi]\right)=\chi(u) \operatorname{tr}\left(\tilde{\beta}_{g}^{*} ; \mathrm{H}_{c}^{0}\left(\tilde{Y}_{\dot{w}}^{m}\right)[\chi]\right)=\chi(u) \operatorname{tr}\left(\beta_{g}^{*} ; \mathrm{H}_{c}^{0}\left(Y_{\dot{w}}^{m}\right)[\chi]\right) .
$$

The right action of $I_{m, w_{m}} / I^{m}$ does not affect the $a$-coordinate of a point $\dot{x} I \in Y_{\dot{w}}^{m}$, thus we see from the Lemma 5.5 that $\operatorname{tr}\left(\beta_{g}^{*} ; \mathrm{H}_{c}^{0}\left(Y_{\dot{w}}^{m}\right)[\chi]\right)=0$, unless $\beta_{g}^{*}(a)=a$. A simple computation 
shows that this can only be the case if $g$ is conjugate to an element in $E^{\times} U_{\mathfrak{J}}^{n}$. This shows (ii)'. If $g$ is conjugate to an element of $E^{\times} U_{\mathfrak{J}}$, then we can assume $g \in E^{\times} U_{\mathfrak{J}}$ and (i) ${ }^{\prime}$ can be shown as in the proof of Proposition 4.22 .

\section{REFERENCES}

[Bo] Boyarchenko M.: Deligne-Lusztig constructions for unipotent and p-adic groups, preprint, arXiv:1207.5876, 2012.

[BH] Bushnell C.J., Henniart G.: The Local Langlands Correspondence for $\mathrm{GL}_{2}$, Springer, 2006.

[BK] Bushnell C.J., Kutzko P.C.: The admissible dual of $\mathrm{GL}(N)$ via compact open subgroups, Ann. of Math. Studies, vol. 129, Princeton University Press, 1993.

[BT] Bruhat F., Tits J.: Groupes réductifs sur un corps local, Inst. Hautes Études Sci. Publ. Math. 41 (1972), $5-251$.

[BT2] Bruhat F., Tits J.: Groupes réductifs sur un corps local II. Schémas en groupes. Existence d'une donnée radicielle valuée., Inst. Hautes Études Sci. Publ. Math. 60 (1984), 197-376.

[BW] Boyarchenko M, Weinstein J.: Maximal Varieties and the local Langlands correspondence for GL $(n)$, to appear in JAMS, arXiv:1109.3522.

[Ch] Chan C.: Deligne-Lusztig Constructions for Division Algebras and the Local Langlands Correspondence, 2014, arXiv:1406.6122.

[De] Deligne P.: La conjecture de Weil II, Publ. Math. IHES, (52):137-252, 1980.

[DL] Deligne P., Lusztig G.: Representations of reductive groups over finite fields, Ann. of Math. (2), 103(1), 103-161, 1976.

[GH] Görtz U., He X.: Basic loci in Shimura varieties of Coxeter type, Cambridge J. of Math. 3 (2015), no. 3, 323-353.

[HV] Hartl U., Viehmann E.: The Newton stratification on deformations of local G-shtuka, J. reine und angew. Math. 656 (2011), p. 87-129.

[Iv1] Ivanov A.: Cohomology of affine Deligne-Lusztig varieties for $G_{2}$, J. of Alg. 383 (2013), 42-62.

[Iv2] Ivanov A.: Affine Deligne-Lusztig varieties of higher level and the local Langlands correspondence for $\mathrm{GL}_{2}$, preprint, 2015, arXiv:1504.00264.

[Lu] Lusztig G.: Some remarks on the supercuspidal representations of p-adic semisimple groups, in 'Automorphic forms, representations and L-functions (Proc. Sympos. Pure Math., Oregon State Univ., Corvallis, Ore., 1977), Part 1, Proc. Sympos. Pure Math., XXXIII, pp. 171-175., Amer. Math. Soc., Providence, R.I., 1979.

[Lu2] Lusztig G.: Representations of reductive groups over finite rings, Represent. Theory 8 (2004), 1-14.

[MSV] Mühlherr B., Struyve K., Van Maldeghem H.: Descent of affine buildings I: large minimal angles, Trans. AMS 366 (2014), 4345-4366.

[PR] Pappas G., Rapoport M., Twisted loop groups and their affine flag varieties, Adv. in Math. 219, no. 1, (2008), 118-198.

[Pr] Prasad G.: Galois-fixed points in the Bruhat-Tits building of a reductive group, Bull. soc. math. France 129 (2), 2001, p. 169-174.

[Ra] Rapoport M.: A guide to the reduction modulo p of Shimura varieties, Astérisque 298 (2005), 271-318.

[Re] Reuman D.: Determining whether certain affine Deligne-Lusztig sets are empty, Thesis Chicago, 2002, arXiv:math/0211434.

[Ro] Rousseau G.: Immeubles des groupes réductifs sur les corps locaux, Thése Université de Paris-Sud Orsay, 1977.

[St] Stasinski A.: Extended Deligne-Lusztig varieties for general and special linear groups, Adv. in Math. 226, no. 3, 2011, 2825-2853.

[Yu] Yu J.-K.: Smooth models associated to concave functions in Bruhat-Tits theory, preprint (2002).

Alexander B. Ivanov, Fakultät für Mathematik der Technischen Universität München - M11,

Boltzmannstr. 3, 85748 Garching, Germany

E-mail address: ivanov@ma.tum.de

URL: http://http://www-m11.ma.tum.de/dr-alexander-ivanov/ 